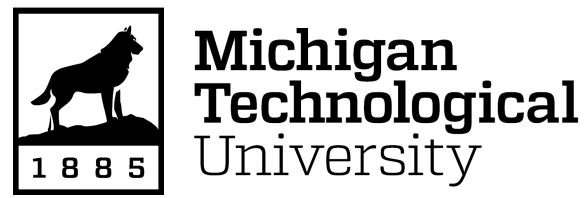

Michigan Technological University Digital Commons @ Michigan Tech

Dissertations, Master's Theses and Master's Reports

2018

\title{
A JOURNEY TOWARDS UNDERSTANDING BIOLOGY HOLISTICALLY AT THE NANOSCALE
}

Trevor Moser

Michigan Technological University, thmoser@mtu.edu

Copyright 2018 Trevor Moser

\section{Recommended Citation}

Moser, Trevor, "A JOURNEY TOWARDS UNDERSTANDING BIOLOGY HOLISTICALLY AT THE NANOSCALE", Open Access Dissertation, Michigan Technological University, 2018.

https://doi.org/10.37099/mtu.dc.etdr/739

Follow this and additional works at: https://digitalcommons.mtu.edu/etdr

Part of the Biophysics Commons, and the Structural Biology Commons 


\title{
A JOURNEY TOWARDS UNDERSTANDING BIOLOGY HOLISTICALLY AT THE NANOSCALE
}

\author{
By \\ Trevor H. Moser
}

\begin{abstract}
A DISSERTATION
Submitted in partial fulfillment of the requirements for the degree of DOCTOR OF PHILOSOPHY

In Biochemistry and Molecular Biology
\end{abstract}

MICHIGAN TECHNOLOGICAL UNIVERSITY

2018

(C) 2018 Trevor H. Moser 
This dissertation has been approved in partial fulfillment of the requirements for the Degree of DOCTOR OF PHILOSOPHY in Biochemistry and Molecular Biology.

$$
\text { Department of Mechanical Engineering - Engineering Mechanics }
$$

Dissertation Co-Advisor: Dr. Tolou Shokuhfar

Dissertation Co-Advisor: Dr. Craig Friedrich

Committee Member: Dr. James Evans

Committee Member: Dr. Ashutosh Tiwari

Committee Member: Dr. Michael Gretz Department Chair: Dr. William Predebon 


\section{Table of Contents}

Acknowledgements ................................................................................................ vi

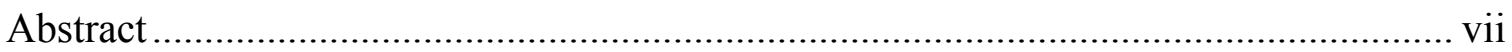

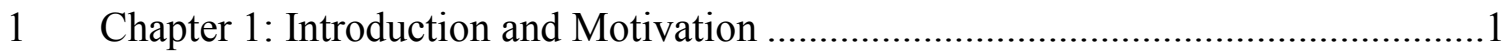

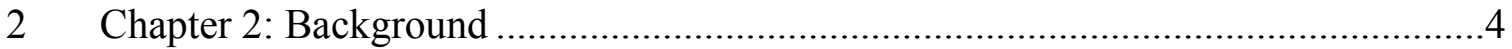

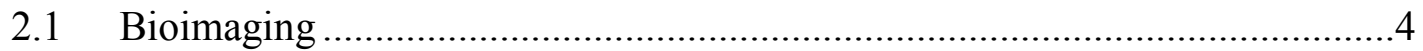

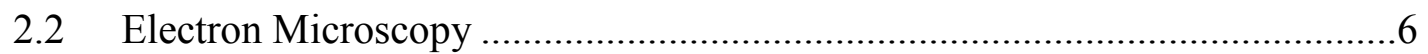

2.2.1 Transmission Electron Microscopy ……………..............................6

2.2.2 Scanning Transmission Electron Microscopy ……………................10

2.2.3 Electron Spectroscopy ………………………..........................12

2.2.4 Cryogenic Electron Microscopy ......................................................14

2.2.5 Liquid Cell Transmission Electron Microscopy .................................15

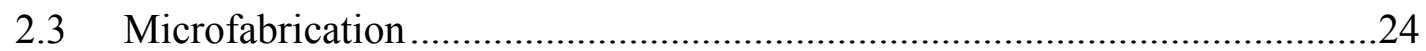

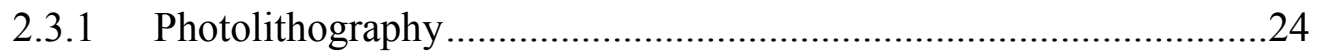

2.3.2 Deposition and Thin Film Patterning.................................................27

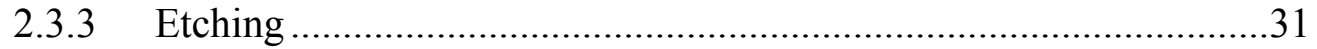

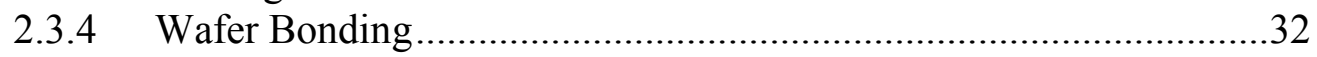

3 Chapter 3: A Holistic Approach to Structural Biology - Characterization at the

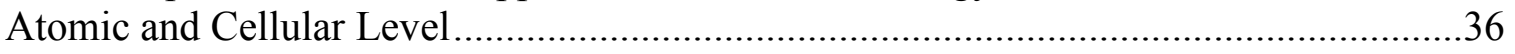

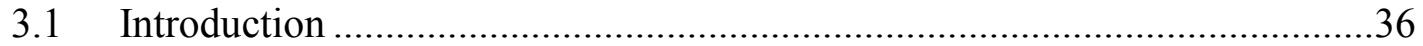

3.2 Cell Free Expression of Soluble Protein for 2D Crystallography ....................36

3.2.1 Cell Free Expression of CcmK .......................................................39

3.2.2 2D Crystallography of $\mathrm{CcmK}$.......................................................42

3.3 Cell Free Expression of Membrane Proteins for Single Particle Analysis.....45

3.3.1 Cell Free Expression of DGAT into Liposomes.................................46

3.4 Correlating Loss of Structural Information Under Electron Irradiation Using

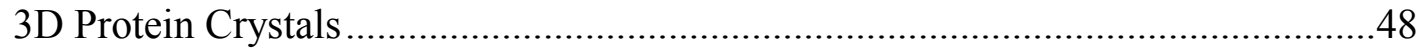

3.4.1 Damage of Catalase with Electron Irradiation...................................50

3.5 Whole Cell Imaging ...................................................................................

3.5.1 Negative Staining and Room Temperature Imaging ……………....52

3.5.2 Cryogenic Electron Microscopy ………………….............................53

3.5.3 Cryogenic Electron Tomography ………………...............................57

4 Chapter 4: Fabrication of Free-Standing Silicon Nitride Membranes for Liquid Cell Transmission Electron Microscopy .................................................................................59

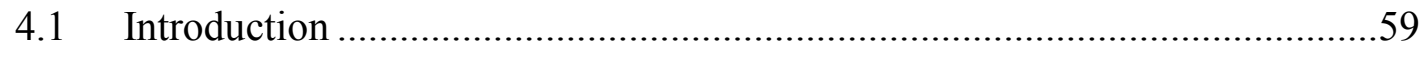


4.2 Design of Nanofluidic Holders and Devices ................................................59

4.2.1 Devices for the Hummingbird LC-TEM Holder ...............................61

4.2.2 Devices for the Protochips LC-TEM Holder ......................................62

4.2.3 Devices for the Dens Solutions LC-TEM Holder...............................64

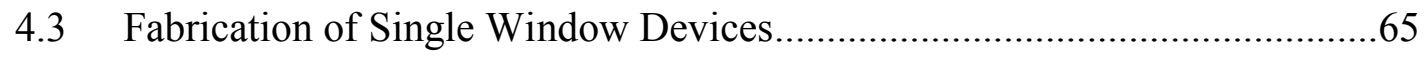

4.4 Using Single Window Devices to Characterize Radiation Induced Chemistry Changes in Liquids

4.4.1 Simulation of Radiolysis Driven Chemistry Changes .......................70

5 Chapter 5: Fabrication of Multiwindow Nanofluidic Devices and the Role of Electron Irradiation History in Interpretation of LC-TEM Results ...................................77

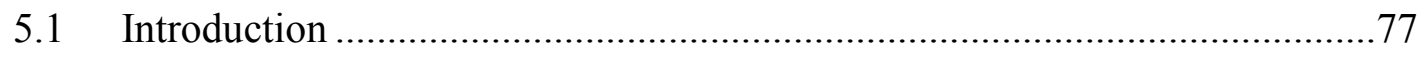

5.2 Limitations of Single Window Devices ........................................................77

5.2.1 Bulging of Membranes ...................................................................77

5.2.2 Limited Imaging Area.....................................................................

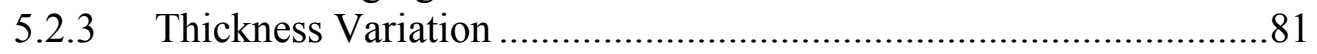

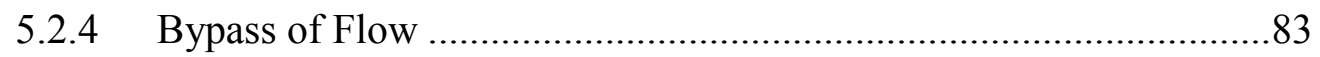

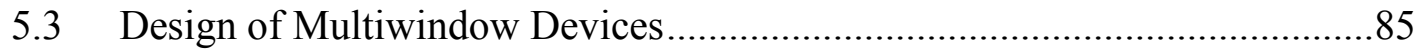

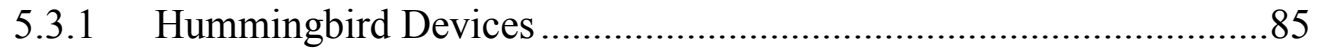

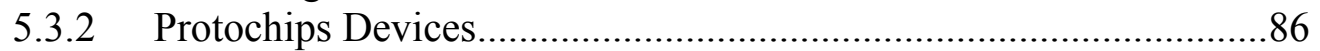

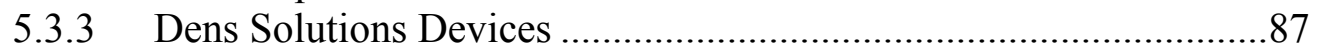

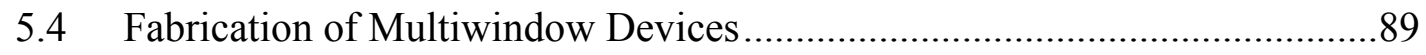

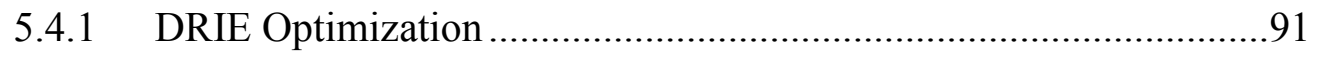

5.4.1.1 Lateral Etch Variation...................................................91

5.4.1.2 Resist Thickness Optimization ........................................92

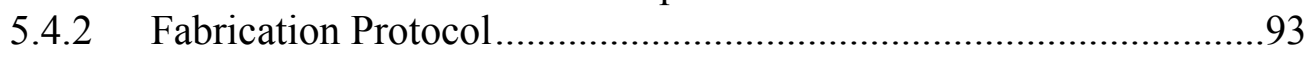

5.4.3 Spacers and Focus Bars ………………….....................................96

5.5 Low Dose Imaging Regime for LC-TEM ………….................................98

5.6 Electron Irradiation History........................................................................101

5.6.1 Lateral Diffusion of Radiolysis Products.........................................102

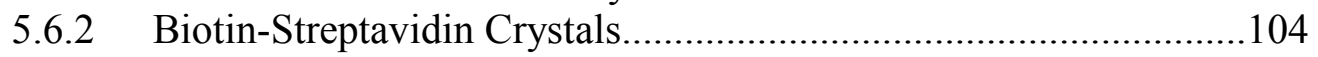

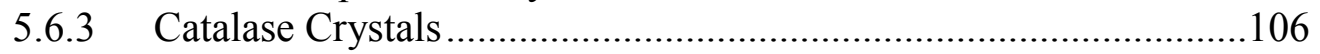

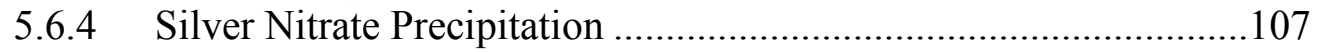

5.7 Imaging Whole Cells with LC-TEM............................................................111

5.7.1 Electron Damage of Whole Cell Structures.......................................113

6 Chapter 6: Nanofluidic Platform for Directed Flow and Enhanced Environmental

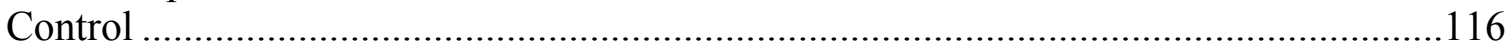

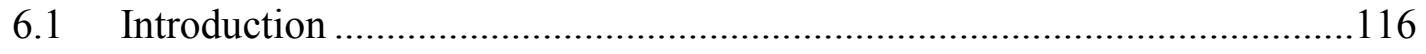

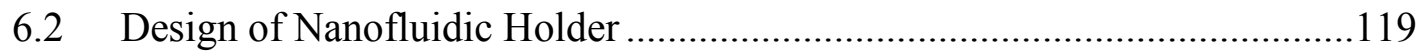




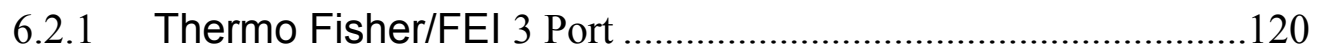

6.2.1.1 Thermo Fisher/FEI Shaft ............................................120

6.2.1.2 Thermo Fisher/FEI Tip ..............................................122

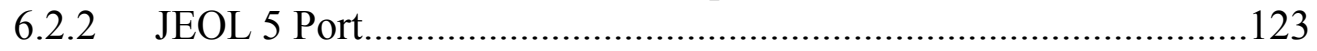

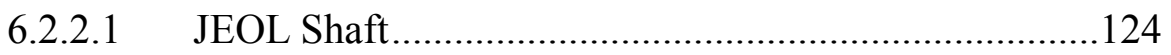

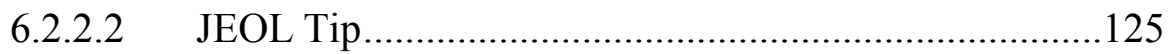

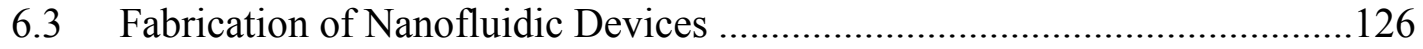

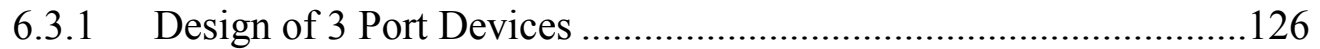

6.3.2 Design of 5 Port Devices ................................................................128

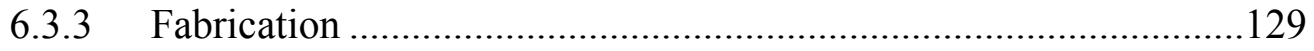

6.3.4 Wafer Bonding ........................................................................131

6.4 Demonstration of Nanoparticle Mixing …………….....................................134

7 Chapter 7: Optimizing Imaging of Low Contrast, Beam Sensitive Samples in LCTEM 137

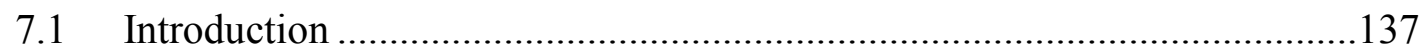

7.2 Comparison of Cryogenic and Liquid Imaging..........................................137

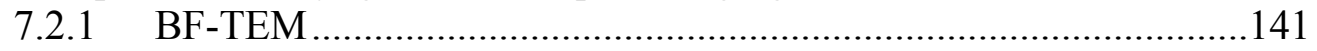

7.2.2 High Convergence Angle STEM ...................................................142

7.2.3 Low Convergence Angle STEM.......................................................142

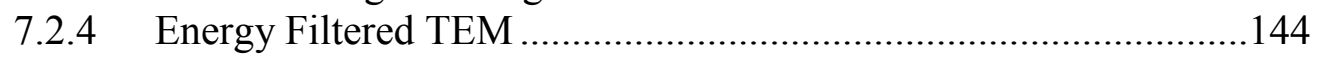

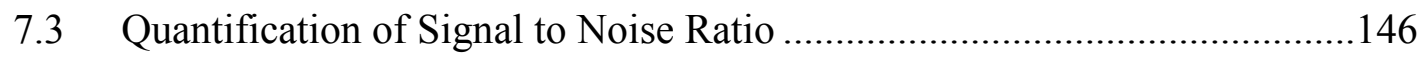

7.4 Electron Irradiation Comparison Between Cryogenic and Liquid Samples 152

$8 \quad$ Chapter 8: Conclusions and Future Outlook.......................................................158

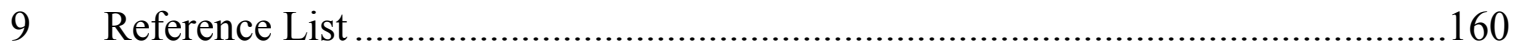




\section{Acknowledgements}

For my parents, without whom this would not have been possible.

I appreciate the opportunity to perform my dissertation work in conjunction with Michigan Technological University and Pacific Northwest National Laboratory (PNNL).

This research was supported by DOE-BER Mesoscale to Molecules Bioimaging Project FWP\# 66382 and the National Science Foundation CAREER award (Grant No DMR-1564950). A portion of the research was performed using the Environmental Molecular Sciences Laboratory (EMSL), a national scientific user facility sponsored by the Department of Energy's Office of Biological and Environmental Research and located at PNNL. 


\section{Abstract}

Characterizing biological processes with microscopy techniques that allow one to directly visualize the complexity of life is an important component of understanding both physiological function and structure. The wide spectrum of biological structures from individual proteins to whole ecosystems necessitates that multiple techniques are used to characterize all levels of organization. While existing techniques cover portions of this spectrum, continued improvement of established methods and development of new techniques is needed. This dissertation outlines my journey in enabling new approaches for imaging biosystems at various scales. Chapters 1 and 2 provide motivation for bioimaging and background for the use of electron microscopy and microfabrication techniques for imaging applications. Chapter 3 highlights my use of established electron microscopy techniques for structural biology. Cell-free expression is described as a biomass production method for generating proteins of sufficient yield and purity for structural analysis. Here I demonstrate a 2D projection map of the protein $\mathrm{CcmK}$ at $14 \AA$ resolution using $2 \mathrm{D}$ electron crystallography as well as the expression of the membrane protein DGAT into liposomes for use in single particle electron microscopy studies. I also present nanoscale cryogenic imaging of whole cells in their native state using electron tomography. Chapter 4 introduces the concept of imaging liquids with electron microscopy and its potential for capturing dynamic processes in biology and chemistry in real-time. Details for fabrication of the devices used for imaging are included. Radiation 
chemistry and numerical simulation is used to predict $\mathrm{pH}$ changes of the solution depending on the solutes present in the aqueous solution. Investigation of the role of electron irradiation effects on liquid samples is continued in Chapter 5 where new multiwindow devices are fabricated and used to characterize the effects of repeated irradiation events on a sample. While the multiwindow devices described in Chapter 5 expand on the scientific utility of the commercially available platforms they are used in, a new platform for directed-flow imaging of liquid and samples in solution with liquid-cell electron microscopy is presented in Chapter 6. This platform allows for increased environmental control, greater experimental reproducibility, and allows for unique mixing and flow experiments not possible prior to the development of this platform. Finally, efforts to optimize imaging conditions for thick, low contrast, and electron beam sensitive samples is presented in Chapter 7 where the image signal-to-noise ratio is quantified across different electron imaging modalities to determine which strategy should be used for optimal liquid imaging. Furthermore, I compare the effects of electron irradiation damage on liquid samples versus cryogenically preserved samples.

Overall, the compilation of research from Chapters 3 through 7 describe findings that provide a basis for future work in advancing the liquid-cell electron microscopy field, from expanding experimental reproducibility, optimizing imaging conditions for future work, and laying the groundwork for establishing irradiation limits for biological structures to enable dynamic imaging at high-resolution and for providing correlative holistic imaging across modalities. 


\section{Chapter 1: Introduction and Motivation}

The wide diversity of life which arises from just four nucleotides is a remarkable example of the hierarchical nature of biology where varied combinations of just a few subunits results in diverse structures and functions. The unique structure of an individual protein is determined by the order of amino acids it is composed of, which in turn is dictated by the genetic code it is translated from. Importantly, the specific structure of a protein is intrinsically related to its function, where this relationship remains important for macromolecular complexes, organelles, cells, tissues, and extends through all levels of biological organization. Understanding the function and structure of biological complexes is the focus of a large amount of biological research, since knowledge of the function of a biomolecule can yield important insights into the mechanisms underlying disease, energy generation, and food production among many others.

Microscopic imaging techniques have been an important technique for biologists since the first cells were imaged with primitive optical microscopes in the $17^{\text {th }}$ century. Since then, visualization of biomolecules has been an integral to understanding and defining the structure function relationship. A well known historical example is the solution of the helical structure of DNA determined by James Watson and Francis Crick after seeing Rosalind Franklind's x-ray diffraction patterns $(1,2)$.

Although a number of different bioimaging modalities exist, each technique only fills a particular role for imaging over a given spatial and temporal range. Since 
biological structures span scales from angstroms to meters the types of techniques needed to cover this whole range is extensive. While large portions of the spatial and temporal spectrum are occupied by established techniques, gaps still remain which need to be filled and improvements to some existing techniques remains necessary. The continuing demand for improved bioimaging capabilities is exemplified by the fact that the 2017 and 2014 Nobel prize in Chemistry were awarded for the development of cryogenic electron microscopy and for the development of super resolution fluorescence microscopy respectively.

Imaging biological dynamics in real time and at high spatial resolutions is an existing gap in the spectrum of bioimaging. While x-ray diffraction techniques can provide subatomic resolution structures of crystalized proteins, and cryogenic electron microscopy can provide atomic resolution structures of proteins and nanometer resolutions of whole organisms, the ability to observe dynamic processes occurring in the same sample in its native hydrated state is not currently possible. Liquid cell transmission electron microscopy (TEM) has the potential to fill this gap. As the field has only relatively recently begun in earnest, in order to realize liquid cell transmission electron microscopy of biological dynamics a number of instrumental and technique improvements are needed. At the offset of this dissertation many challenges existed for imaging cells in liquid within a TEM. Better design and fabrication of platforms for holding liquid samples inside the microscope were required, along with microfabrication work to 
allow for improved environmental control of samples and enhance reproducibility. Characterization of imaging artifacts due to electron beam driven morphological and physiological changes were required for confidence in observed results, and the parameters for obtaining images which yield the most information was still unknown. The following chapters detail my efforts and journey to become an expert in TEM at room and cryogenic temperatures, experimental biochemistry and molecular biology and advance liquid cell transmission electron microscopy for biological samples through instrumentation and technique development. 


\section{Chapter 2: Background}

\subsection{Bioimaging}

The wide range of imaging strategies for investigation biological structures testify to the large scale of structural information contained within biological systems. The sensitivity of biological structures to environmental conditions such as temperature, light, humidity, and chemical/mechanical stimulus requires that imaging is performed in a way that minimizes physiological perturbation of the structures and processes being visualized. As such, techniques where the probe does not damage the sample, or in the case of damaging techniques, the sample is stabilized in a manner that slows or reduces the damaging effect of the probe.

The use of visible light is perhaps the most widely utilized method for bioimaging, where photons with wavelengths of 400 to $700 \mathrm{~nm}$ pass through the sample to transmit information onto a CCD or the eyes of the observer. The popularity of visible light microscopy (VLM) stems from its general ease of use and photons within the visible range are widely considered to be "non-damaging" to biological samples. Labeling is possible through the use of fluorescent probes (3), and fluorescent protein fusions can allow for tracking single molecules $(4,5)$ or the expression of protein copies produced from an organism (6-8). In these cases however, the molecule or protein of interest must be known and available for targeting by a label. Label free imaging of biological structures can be achieved through Raman microscopy techniques, where the specific vibrational state of the molecule of interest can be used as a source of signal for imaging $(9,10)$. A 
disadvantage of the use of photons in the visible range is the diffraction limited resolution of the technique to approximately $200 \mathrm{~nm}(11,12)$. Instrumentation and computational advances have resulted in so called "super-resolution" VLM techniques where the positions of fluorophores can be determined, in some ideal cases, to below $20 \mathrm{~nm}$ spatial resolution (13-16).

To overcome resolution limitations of visible light, the wavelength of the photons used can be lowered to reduce the diffraction limit of the technique. The use of $x-$ rays have a long history of use for imaging biological structures, and are typically broadly classified as soft (2-4 $\mathrm{nm}$ ) or hard (less than $1 \mathrm{~nm}$ )depending on their wavelength (17). Soft x-ray microscopy and tomography has been used to image whole cells in liquids and ice microns in thickness and with tens of nanometers spatial resolution (18-22). Hard x-rays are most commonly used in crystallography, representing by far the bulk of solved protein structures (23-25). X-rays however are ionizing radiation sources and will damage biological structures (26). Ultra-bright x-ray sources have been used in so called diffract before destroy experiments but are intrinsically destructive and cannot visualize dynamics (27-29).

Other modalities outside photons can be used for imaging, such as the use of ions in secondary ion mass spectrometry (SIMS) (30). In this technique ions are used to sputter fragments of the sample of interest which is delivered to a mass spectrometer to gain chemical information about the sample $(31,32)$. Other techniques for imaging biological samples include atomic force microscopy (33), 
neutron scattering (34), and nuclear magnetic resonance (35). Imaging with electrons is also possible and has recently gained a large amount of interest for imaging biological samples.

\subsection{Electron Microscopy}

A wide range of imaging strategies exist for imaging biological samples from the atomic to multicellular scales. However, no one imaging technique is capable of characterizing structures and phenomena at every spatial and temporal scale. Rather, each technique typically covers one part of the landscape, where techniques often overlap each other to some degree. Classically, the use of high energy electrons to form projection images of thin samples provides images with spatial ranges from angstrom to microns, and temporal resolutions of milliseconds and greater. For biological applications, transmission electron microscopy has filled a niche for angstrom and nanometer level interrogation of proteins and cell ultrastructure (36-40).

\subsubsection{Transmission Electron Microscopy}

Generation of electrons from cathodes in vacuum tubes has been known since the late 1800 s, although the development of electromagnetic lenses for focusing were not developed until the first generation of electron microscopes in the 1930's (41-43). Modern day transmission electron microscopes (TEMs) generate electrons from $30 \mathrm{kV}$ to $300 \mathrm{kV}$ and can contain advanced optics for aberration correction allowing for atomic scale imaging of ideal samples (41). A general 


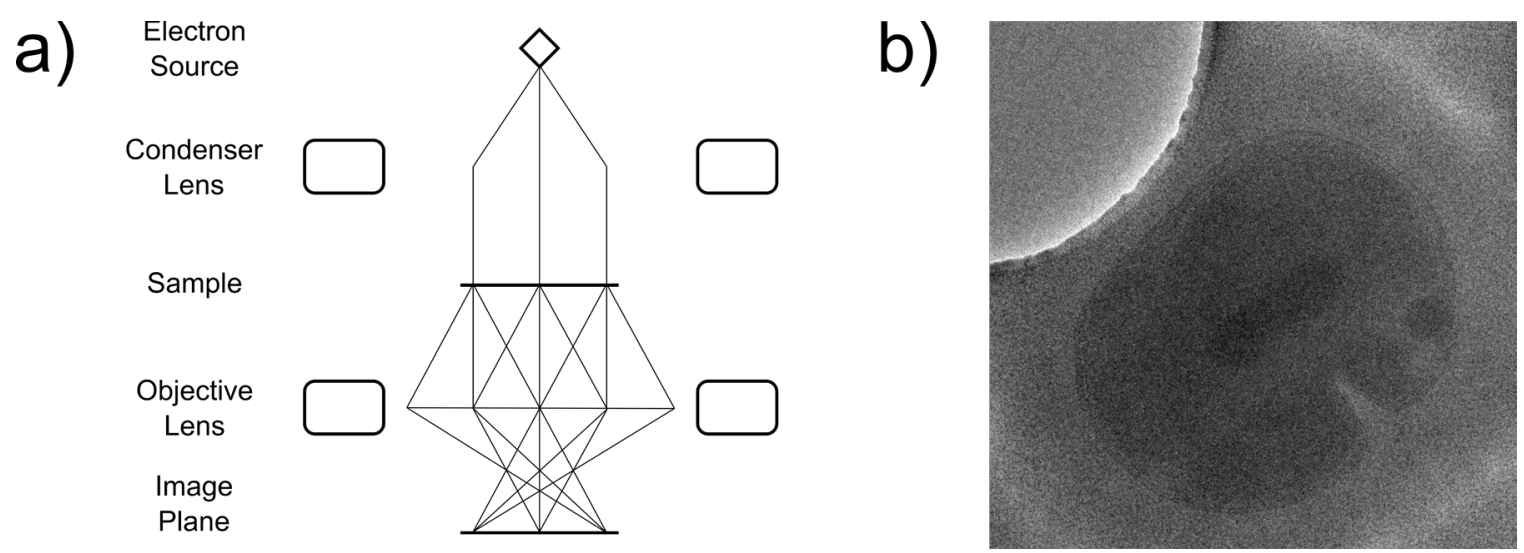

Figure 2.1: a) Simplified illustration of a transmission electron microscopy depicting the route of electrons as they are emitted from the electron source and pass through a sample and transmit information to the image plane. b) A phase contrast transmission electron microscope image of $O$. tauri imaged with cryogenic electron microscopy.

overview of a TEM is shown in Figure 2.1a detailing the focusing of electrons onto a sample and image formation on a detector below the sample. As electrons interact strongly with matter the inside of the microscope column must be maintained at high vacuum to prevent extraneous scattering of electrons with gas molecules (41). A series of mechanical and ion pumps are used to maintain the vacuum of the column around $10^{-6}$ mbarr at all times (41). Additionally, physical lenses cannot be used for electron focusing as they would absorb the electrons as they pass through. As a result, electromagnetic lenses are used which create an electric field. Electrons traveling through these fields experience a Lorentz force which can focus the electron beam by tuning the strength of the electric field (41). For TEM imaging, condenser lenses above the sample focus the electrons onto the sample as a broad, parallel illuminating beam. An objective lens after the sample focuses electrons scattered by the sample and projection lenses focus focused electrons onto a viewing screen or camera at the desired 
magnification, either on the image plane for real space imaging or the back focal plane for diffraction imaging. Alternatively, a convergent electron beam may also be used to perform scanning transmission electron microscopy. The image formation mechanism in this strategy is different and will be discussed in the next section.

When electrons transmit through a sample they can interact, or scatter, with the atoms of the sample as they pass through. Whether these interactions happen is probabilistic, where the chances of a collision is proportional to the atomic cross section (41). While the factors affecting the cross section of a particular sample are complex, it can be simply approximated to be related to the energy of the electron (in $\mathrm{eV}$ ), the charge of the electron, the scattering angle, and the atomic number of the atoms of the sample (41). Increasing the energy of the electron decreases the scattering cross section, while increasing the atomic number of atoms in the sample increases the scattering cross section. Coulomb interactions between the negatively charged electrons, and the negatively charged electrons in atomic orbitals or the positively charged nucleus of the atom dictate the scattering interactions of the beam electrons and the atoms in the sample (41). Scattering events can be broadly defined as elastic collisions (no energy transfer) or inelastic collisions (transfer of energy from the incident electrons to the sample). Elastic collisions are typically of most interest for image formation with biological samples as inelastically scattered electrons become incoherent and do 
not contribute to constructive and deconstructive interference at the image plane during phase contrast imaging (41).

Phase contrast imaging entails collecting both the high angle elastically scattered electrons and transmitted electrons that do not interact with the sample.

Electrons which have scattered experience a phase shift where the magnitude of this shift is dependent on the angle and type of scattering. When the objective lens focuses scattered and transmitted electrons back to the same place on the image plane contrast forms as a result of constructive and deconstructive interference of the phase shifted electrons with incident transmitted electrons. While this technique is advantageous as it uses all of the signal from elastically scattered electrons, the magnitude of phase shift is related to the atomic species and density of the sample. Because biological samples are primarily composed of low z number atomic species they are generally weakly scattering. As a result, images of biological samples often have very low inherent contrast in phase contrast images, as shown in Figure 2.1b. The recent implementation of reliable phase plates, which induce an increased phase shift in scattered electrons, can significantly increase the contrast of biological samples in phase contrast imaging although they are not yet widely available in all microscopes $(44,45)$. The imaging performed in subsequent chapters of this dissertation show results which, unless otherwise noted, do not utilize such phase plate technology. Images are ultimately focused by the objective lens onto the image plane. Recording images was historically performed with film and then charge coupled 
device (CCD) cameras. Currently CCDs are the most ubiquitous method for image collection, where electrons are collected on a scintillator which converts the signal to photons which are then detected by the CCD. Because of this conversion ( $\mathrm{e}^{-}$to photons to $\mathrm{e}^{-}$) a large amount of signal is lost and noise is introduced into the final image. Recently, developments of direct electron detectors (DEDs) which directly count electrons at the image plane have considerably improved signal collection efficiency and improved image signal to noise and contrast (46). While not yet common on all microscopes, their use is becoming increasingly common especially for structural biology (46).

\subsubsection{Scanning Transmission Electron Microscopy}

While TEM uses a broadly illuminating beam to collect an image, the electron beam may also be focused to a small convergent probe which is the scanned across an area of the sample being imaged (47). This technique is known as scanning transmission electron microscopy (STEM), where an overview of the lens system and path of electrons is shown in Figure 2.2. Condenser lenses before the sample focus the electron beam into a convergent probe, generally around $2 \AA$ in diameter or less $(48,49)$. Scattered electrons through the sample are collected in bright field detectors (transmitted and low angle scattered electrons), and annular dark field detectors (electrons scattered at larger angles) (48). For each position that the STEM probe is positioned the signal hitting these detectors is integrated and plotted as an intensity value. This is repeated for 
a)

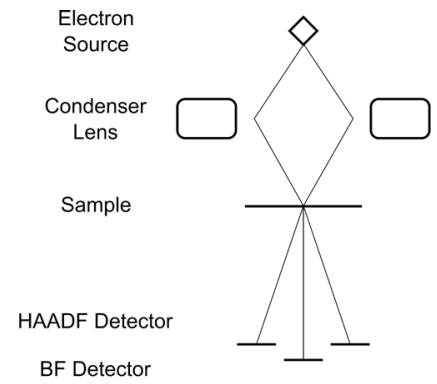

b)

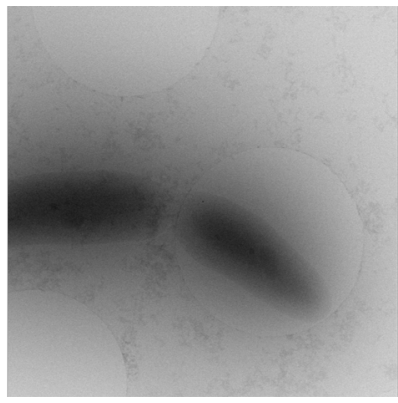

c)

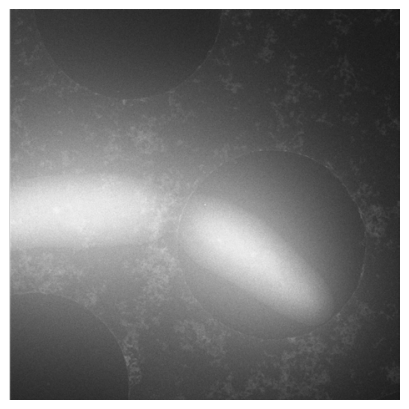

Figure 2.2: a) Simplified illustration of a scanning transmission electron microscope depicting the lens system used to project the beam onto the sample and to the detectors below the sample plane. b) Bright field scanning transmission electron micrograph of $C$. metallidurans in a layer of vitrified ice. c) Complementary high angle annular dark field scanning transmission micrograph of C. metallidurans in a layer of vitrified ice.

every pixel position that the STEM probe dwells at as the beam is rastered across the sample. An advantage of STEM imaging is that incoherently scattered electrons which are scattered to large angles can be detected in a high angle annular dark field (HAADF) detector, where the intensity of the signal is directly related to approximately the square the $z$ number of the atomic species being imaged. In this way, so called "z-contrast imaging" can be performed (48). Because the dark field detectors are annular in shape, both dark field and bright field information of a sample can be collected simultaneously. Figures $2.2 \mathrm{~b}$ and 2.2c show BF and HAADF images of biological cells imaged with STEM depicting the contrast that can be obtained with the technique.

While biological samples are classically imaged with TEM, advantages of STEM imaging have been increasingly discussed (50-53). In particular, advantages of low convergence angle BF-STEM for very thick, low contrast samples such as cells has been reported (54). 


\subsubsection{Electron Spectroscopy}

While the inelastic collisions some electrons experience are not always desirable since they contribute to the noise signal of the image and are responsible for secondary damage events, they generate valuable information that, if captured correctly, can yield information about the atomic species the collision happened with. During an inelastic collision, the energy transferred to one of the electrons in the atoms orbitals causes it to become excited to a higher energy state or to become ejected entirely. When another electron drops in energy to fill the hole left over energy is given off which is proportional to the energy difference between the orbital hole and the original orbital of the decaying electron. This energy is given off in the form of an x-ray, where the energy and wavelength of the emitted x-ray corresponds directly to the atomic orbital the inelastic collision originally occurred at. The collection of x-rays emitted during electron irradiation is known as energy dispersive $\mathrm{x}$-ray spectroscopy (EDS), a technique that can map the atomic composition of the sample.

Alternatively, the energy lost by the incident electron during an inelastic collision is also directly related to the atomic orbital from which the collision occurred at. By determining the energy lost by an electron relative to the energy of the incident electrons the elemental composition of the sample can be identified. Because electron detectors are not directly capable of quantifying the energy of an electron, the transmitted electrons are separated with an electromagnetic prism prior to collection on a CCD, shown in Figure 2.3a. Electrons are focused 

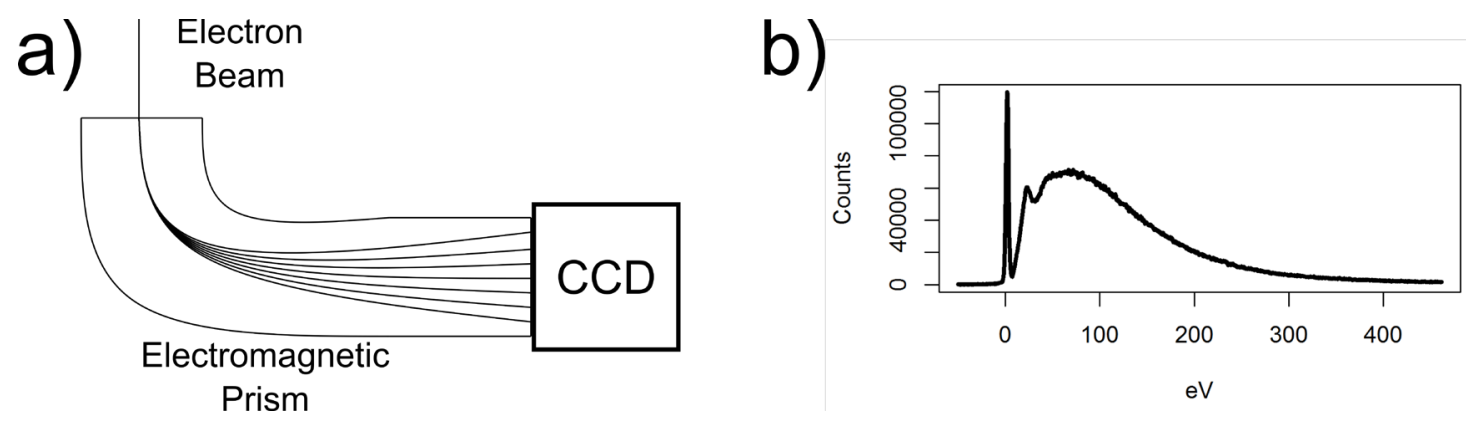

Figure 2.3: a) Simplified illustration of an electron spectrometer depicting the electromagnetic prism used to separate electrons of differing energies onto a CCD detector. $b$ ) Typical electron energy loss spectra depicting the zero loss peak and plasmon.

at different strengths through the electromagnetic field, where higher energy electrons have a larger radius and lower energy electrons have a smaller radius (55). The spectrum, shown in Figure 2.3b, on the CCD is normalized to the zero loss peak (elastically scattered electrons), where the energy loss of the remaining electrons in the spectrum can be determined relative to this zero loss peak, in a process known as electron energy loss spectroscopy (EELS). While elemental mapping of samples is possible with EELS and has been demonstrated with biological samples $(56,57))$, the technique may also importantly be used to estimate the thickness of a sample. Because the probability of an inelastic event is a function of the thickness of the sample, by integrating the signal from the zero loss peak (elastic collisions) and the rest of the signal from the spectrum (inelastic collisions), the thickness in inelastic mean free paths, or the average number of inelastic collisions an electron experiences traveling through the sample, can be determined (55). If the general composition of the sample is known, the inelastic mean free path can be estimated, and used to quantify the thickness of the sample in nanometers (58). 


\subsubsection{Cryogenic Electron Microscopy}

Because biological samples are dependent on their hydration for both their structure and function, steps for preventing the dehydration of the sample must be taken when imaging with TEM. One method for achieving this is to freeze the and maintain the sample at cryogenic temperatures so that the ice does not sublimate in the column. This is commonly known as cryogenic electron microscopy (cryo-EM) (59-61). To prevent the formation of ice crystals during freezing which would damage biological structures the sample must be cooled at a very high rate to achieve vitrification, producing amorphous ice surrounding the trapped sample $(59,62)$. This is done by rapidly plunge freezing the sample in liquid ethane cooled well below its boiling temperature to minimize insulating effects from the Leidenfrost effect (59). In recent years cryo-EM has gained interest and popularity in conjunction with the development of direct electron detectors which drastically improve the contrast and signal to noise ratio of biological samples. The structures of difficult to crystalize proteins have been determined with single particle imaging techniques and the structure of macromolecular complexes have been solved using in situ cryo-EM tomography and subtomogram averaging of whole cells (63).

While cryo-EM techniques have gained enormous popularity in recent years for its ability to resolve the structure of proteins $(64)$, viruses $(65,66)$, and whole cells (67) at high spatial resolutions, it is mostly limited to observing static processes. Time resolved studies can be performed by stimulating a reaction and 
freezing samples at different time points within the dynamic process (39), but high temporal resolutions are not possible and observations of physiological behavior occurring in the same cell or sample are not possible. As such there is a need for an imaging technique which provides high spatial and temporal resolution for samples within their native hydrated state.

\subsubsection{Liquid Cell Transmission Electron Microscopy}

Imaging liquids in a transmission electron microscope poses unique challenges to maintaining the integrity of the liquid sample under the high vacuum environment of the column. Typically, strategies for imaging liquid or humid samples entail either trapping the sample within a hermetically sealed chamber containing electron transparent windows or using a differential pumping system to allow for higher pressures around the sample region compared to the rest of the column (68). The first demonstrations of both of these techniques were demonstrated over 70 years ago, demonstrating the potential for the technique $(69,70)$. However, limitations with instrumentation limited the scope of questions which could be answered, and it was not until advances in microfabrication techniques allowed for the production of uniform, very thin free standing

membranes that the potential of imaging liquids in hermetically sealed environments was realized (71). Additionally, advances in vacuum technology has improved the ability to maintain high pressure regions within the otherwise ultra-high vacuum environment of the TEM column, now commonly referred to as environmental transmission electron microscopy (ETEM) $(68,71,72)$. While 
ETEM has been used successfully to image hydrated, or humid, samples the pressures available are only in the mbar range (72). As the physiological and morphological behavior of biological samples are intrinsically related to their level of hydration, a technique which maintains the complete hydration of the cell or protein is preferable. As such, while ETEM can be used to image biological samples it seems advantageous to use a closed cell system for imaging cells, proteins, and complexes.

A number of different closed cell systems have been developed which may be broadly classified as either static or dynamic flow cells. Static cells trap a liquid sample between two electron transparent membranes, and achieve hermeticity by completely sealing the liquid volume within a chamber. The earliest demonstration of a static liquid cell used nitrocellulose films as the electron transparent membrane (70). With the reintroduction of the technique in the early 2000s (71) the most commonly used electron transparent membrane material is silicon nitride, used for its ease of fabrication on thick silicon support substrates. A number of various systems utilizing silicon nitride membranes on a silicon substrate in a closed static liquid cell system have been described in literature with applications in cell biology (73-75), electrochemistry (76), and nanomaterials (77). Sealing of these devices is often achieved using an adhesive to glue the upper and lower substrates two each other, forming the liquid volume. Other unique solutions for trapping liquids entail creating chambers within the silicon nitride itself $(78,79)$, or within channels formed within a channel entirely within a 
silicon nitride film $(80,81)$. The inability to exchange the liquid sample, whether introducing a stimulating reagent to trigger a reaction or physiological change or replacing media as components are consumed by growing cells, limits the scope of science which can be done with these devices. In particular for whole cell imaging, the rapid depletion of the surrounding media and buildup of toxic metabolic byproducts can result in organisms entering a physiological state that is not representative of native conditions.

The most common static cell regime outside these silicon nitride liquid cells is the use of graphene to trap small volumes of liquid between sheets of graphene (82). The pi bond stacking and van der Waals between the adjacent sheets is strong enough to hermetically seal the liquid sample trapped between the layers (83). These so-called graphene liquid cells have been used to image nanomaterials $(82,84-86)$ in addition to biological samples included cells and protein complexes (87-90). Graphene is advantageous as the atomically thin layers provide very little extraneous electron scattering when compared with thicker silicon nitride membranes, improving signal to noise, contrast, and resolution. Furthermore, the potential for the graphene to act as a radical scavenger and somewhat reduce the effect of radiolysis effects in a liquid sample has been proposed (91). While the fully effects are not well known, effects of a confined environment on biological samples may have an effect on the organisms growing within it. The inability to flow fresh, stimulating media or sample to initiate a dynamic reaction is also difficult or not possible with graphene liquid cells. 
Dynamic flow holders are similar to static systems which use silicon nitride membranes on a silicon support to hermetically seal the liquid sample, but also include microfluidic tubing and channels to allow for the delivery of liquid sample to the devices being imaged. Commercial solutions for these holders exist, where flow is often delivered to the tip of a TEM holder where it fills a hermetically sealed chamber. Some of this liquid then finds its way in between the nanofluidic devices containing the electron transparent membranes so that imaging may be performed. Other custom build solutions also exist, often designed so that flow is channeled directly into a nanofluidic channel through vertical flow channels on the bottom of the devices (92-95). The advantage of these designs is that the liquid sample is forced to flow directly in between the devices and to the imaging region. In other regimes a considerable bypass channel which can be microns in width exists allow for sample to flow around, rather than through, the imaging devices. However, holders utilizing this design are not currently available commercially, and their implementation remains challenging and requires difficult fabrication and wafer bonding techniques. As such, the majority of existing literature on LC-TEM has been published with commercially available solutions which deliver liquid sample to the hermetically sealed chamber containing the nanofluidic devices, some of which finds its way in between the devices and to the imaging area and some of which flows around the devices entirely and flows out the exit port for the holder. 
While the applications of LC-TEM cover a broad range of applications, the characterization of nanomaterials and the nucleation of nanoparticles represents perhaps the bulk of published literature with the technique (96-101). One side effect of the high energy electrons used for image formation is inelastic scattering events which transfer energy to the sample being imaged. These energy transfer collisions result in bond breaking (radiolysis) and the ejection of orbital electrons from their shells, known as secondary electrons (41). In liquids, the bond breaking of water molecules during electron irradiation causes the formation of highly reactive radical species $(102,103)$. These radical species can then reduce metal salts in solution, causing the nucleation and growth of nanoparticles (97). Using the electron beam as a "reducing agent" has been used to study both the underlying mechanisms of nucleation and growth kinetics, but also to understand the chemistry changes of liquid samples as a result of radiolysis $(101,104,105)$. In addition to the precipitation of particles, electron beam driven chemistry changes in a solution has also been shown to cause dissolution of particles via radiolysis induced chemical etching (106-108). LC-TEM has also been used as a characterization tool for organic based materials such as metal organic frameworks and polymers $(109,110)$.

LC-TEM has also been demonstrated for biological samples from proteins (111$114)$ to whole cells $(115)$. Both prokaryotes $(74,116,117)$ and eukaryotes $(118)$ have been imaged with LC-TEM, and although mammalian cells have been imaged the large fluid thicknesses necessitated by their size results in images 
which are low in contrast (119-121). As electron beam damage is a concern for biological samples, many reports of cells imaged with LC-TEM are fixed with a crosslinking agent such as glutaraldehyde in order to stabilize the cell from the effects of secondary damage (121). Due to the low intrinsic contrast of whole cell samples and the compact nature of proteins within the cell, identifying the positions of biomolecules of interest requires some labeling with a reporter that provides high contrast. This has been demonstrated with LC-TEM using antibody functionalized with quantum dots or nanoparticles $(74,121)$. Quantum dots have some advantage as they allow for the potential for correlative fluorescence imaging, although labeling is generally constrained to membrane proteins which have epitopes for antibody binding located outside the cell. Attempts to negative stain biological samples in liquids has also been described (117), although the viability of cells under these conditions is the subject of some disagreement (122, 123).

While LC-TEM holds great potential for visualizing dynamics of biological processes at high resolutions, a number of factors constrain the scope and application of the technique. Several of these limitations are a product of the design and application of the instrumentation used to hold the liquid samples, while the physical interaction of high energy electrons with the sample and the uncertainty of the fate of damage byproducts confounds interpretation of observed dynamics. Instrumentation limitations include limited imaging area, thickness variability, and bypass of flow. Typical commercially available 
nanofluidic devices contain a single silicon nitride window, which when assembled in the holder with a sample results in an imaging region which can be $30 \times 30$ um to $50 \times 50$ um depending on the width of the windows (75). The silicon nitride membranes used for imaging are typically $10-50 \mathrm{~nm}$ in thickness and have a high degree of flexibility $(75,78)$. When placed inside the column of a TEM the pressure differential between the column (generally around $10^{-6} \mathrm{mbarr}$ ) and the liquid sample (at atmospheric pressure) results in a significant outward bulging of the silicon nitride membranes and can be as much as $500 \mathrm{~nm}$ to $1 \mathrm{um}$ in the center of the imaging area (124). As a result, imaging is practically limited to the corners of the electron transparent membranes, further reducing the area available for performing imaging experiments in. As a result of the limited area for imaging, in order to perform experiments to generate statistics and demonstrate reproducibility multiple samples must be assembled. While spacer material patterned on the surface of the devices can be used to define the minimum thickness of the liquid volume (75), in practice the actual liquid thickness has a tendency to be highly variable. Environmental contaminants from the sample or out of the air can rest on the surface of the device, and if large enough can dictate the minimum thickness of the fluid cell. The sample itself can additionally cause thickness variability if the sample is nonhomogeneous and have a tendency to clump together. Clumping and variable cells sizes are typical of biological samples, and duplication of liquid thicknesses tend to rely more on luck than skill as the devices are currently designed. 
Finally, while most commercial holders allow for the flow of fresh sample or solution to the imaging area, the introduction of samples such as cells or particles to the imaging area is complicated by a large flow bypass channel caused by the geometry of the devices. While the spacing between the devices is typically at maximum 1-2 um and ideally closer to several hundred nanometers, the channel around the devices between the sealing o-rings and the walls of the holder tip that allows for liquid flow can be tens to hundreds of microns in width and depth. In addition to limited how samples can be loaded in the devices (large samples cannot be practically flowed into the imaging area) it constrains the ability to perform dynamic experiments such as the growth or coalescence of nanoparticles from the mixing of two separate sample solutions. While some commercial holders claim to have the ability to flow multiple samples from two inlet lines they combine just before they enter the hermetically sealed chamber (125). Experiments utilizing these holders are not able to visualize true time zero nucleation events, and uncertainty about "filtering" effects of the bypass channel are unknown as the liquid from this channel has not been analyzed for any of these experiments (125). Overcoming these design limitations will require redesigning the holders and the devices used for LC-TEM imaging.

In addition to the geometric limitations imposed by the instruments used with LCTEM, the physical interaction of the electron beam with the liquid sample can create image artifacts and confounds interpretation of experiments and images. When liquids are irradiated with high energy electrons some of the energy is 
deposited into the sample during inelastic collision events. These collisions can eject orbital electrons and break atomic bonds through radiolysis (102). The secondary electrons ejected into the sample, although lower energy, can also undergo further inelastic collisions and continue to break atomic bonds. For pure water, primary radicals are formed which then react with each other and other water molecules and reach a steady state equilibrium where the concentrations of these radicals is dependent on the incident dose deposited by the electron beam (102). These radical species are:

$$
\mathrm{H}_{2} \mathrm{O} \rightarrow \mathrm{H}_{3} \mathrm{O}^{+}, \mathrm{OH}^{-}, e_{a q}^{-}, \mathrm{H}^{-}, \mathrm{OH}^{-}, \mathrm{H}_{2} \mathrm{O}_{2}, \mathrm{H}_{2}, \mathrm{HO}_{2} \text {. }
$$

The rate of production of these species during irradiation is dependent on the so called G-value for each species, defined as the rate of generation per $100 \mathrm{eV}$ of deposited energy (102). While real samples are typically more complex containing solutes of different molecular species, as long as the sample is at low concentrations it is generally considered that the bulk of radicals are from the solvent (102). In the simple case of a metal salt, these radical species can reduce the solute ions in solution, which can then precipitate depending on the chemistry of the solution (126). Understanding the interaction of these radical species with the sample is critical for accurate interpretation of LC-TEM results. While not well understood, the charging of the silicon nitride membranes under electron irradiation has also been discussed as a damage mechanism for liquid samples (127). Especially for biological samples for which the radical species can react strongly with proteins, DNA, and other biomolecules to alter the 
organism or structure from its native state, the role of electron irradiation on chemistry changes in the sample must be considered when attempting to observe physiological behavior.

\subsection{Microfabrication}

While microfabrication techniques have had perhaps the greatest impact on the semiconductor industry for manufacturing computer chips and processors, it is also used to great effect for creating microfluidic and microelectromechanical (MEM) devices (128). These devices often have features or structures at the micron to nanometer scale, where the microfabrication processing allows for a high degree of control and reproducibility (129). Applications of these devices are broad including sensing pressure $(130,131)$, orientation (132), or biological molecules (133-136). In addition to sensing applications microfabrication can be used to generate microfluidics with channels of reproducible sizes and shapes $(137,138)$. Fabrication typically entails repeating steps of patterning, etching, and bonding to create the desired design (129). Microfabrication is typically performed in a clean room to reduce the amount of environmental contaminants and improve reproducibility of each microfabrication step (128).

\subsubsection{Photolithography}

Creating reproducible designs across a large number of devices with high throughput requires a reliable method for accurately producing the same pattern. Photolithography is a patterning method which utilizes ultraviolet (UV) light to 
pattern an organic polymer, known as a photoresist, with the desired design using a photomask (129). The basic principle of photolithography is shown in Figure 2.4, highlighting exposing, soft baking, and developing the photoresist on a silicon wafer (the most typical substrate used in microfabrication).

The first step of photolithography is to ensure the substrate surface is clean and passivated for proper adhesion of the photoresist. Cleaning can be performed with a strong oxidizer to remove metal and organic contaminants, followed by a bake at temperature to drive off adsorbed water (129). Depending on the application of the devices the substrate can also be etched in hydrofluoric acid $(\mathrm{HF})$ to remove the native oxide layer that naturally forms on the surface of silicon. Often substrates are spin coated with a thin layer of hexamethyldisilane, which ensures the surface of the substrate is hydrophilic for optimal resist adhesion (128).

After cleaning the photoresist can be coated onto the substrate, usually through spin coating. This involves spinning the substrate at high speeds with the photoresist on the surface, where the resist is spread evenly across the substrate surface (128). Thickness of the resist layer is dependent on the viscosity of the resist and the speed the substrate is spun at during coating and can be tuned 


\section{UV Source}

a)

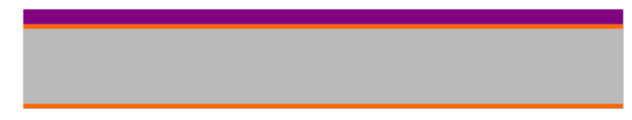

c)

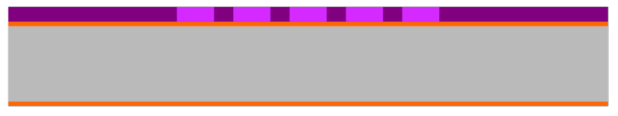

b)

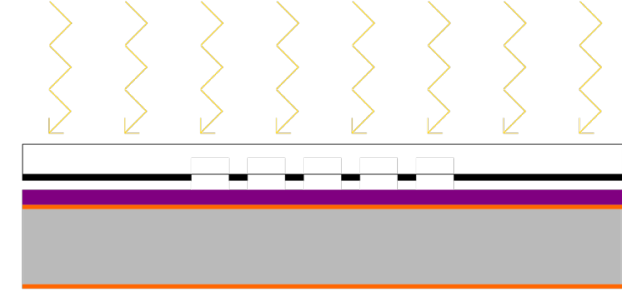

d)

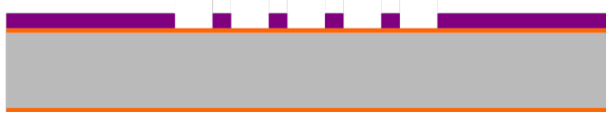

Figure 2.4: a) A photoresist layer (purple) is deposited on the surface of the wafer (orange and grey) through spin coating. b) A photomask is aligned over the wafer and the photoresist is irradiated with ultra violet light. Regions of the photomask with chromium (black) block the ultraviolet light from crosslinking the photoresist in specified areas. c) Crosslinked photoresist (light purple) from ultraviolet exposure may be targeted with a developer. d) After developing resist features are left behind on the wafer.

based on the needs of the devices being fabricated (128). After the resist is layered on the resist it must be soft baked to drive off the solvent in the photoresist. The time and temperature of this soft bake is dependent on the amount of solvent in the photoresist and the thickness of the resist, and is generally done at temperatures less than $120^{\circ} \mathrm{C}$ which is the temperature that polymerization of most photoresist occurs at (128). The resist must then be left to rest at room temperature, as swelling often occurs as the resist rehydrates based on the relative humidity of the clean room (128).

Patterning of the resist is done by aligning a photomask with the substrate and exposing with UV to crosslink the photoresist (128). Photomasks are typically designed using computer aided design software and printed using a system which can directly print patterns onto a photoresist (128). The photoresist on the 
mask is developed exposing a layer of chrome, which is etched away using a chrome etchant (128). The photomask is then used to copy the design to the layered photoresist on the desired substrate, where UV exposure on the substrate photoresist only occurs where the chrome in the photomask has been etched away, allowing for transmission of UV to the photoresist. Photoresist that has been exposed by UV becomes crosslinked which defines the region of the photoresist which is removed during the development step (128).

Photoresists can be classified as positive or negative, where crosslinked photoresist is removed in positive resists and non-crosslinked photoresist is removed in negative photoresists (128). Figure 2.4 is an example of a positive photoresist. Developers are typically specific to the photoresist, positive or negative, that is being developed where the developing time and resolution is dependent on the thickness and type of resist used. After developing the photoresist it can also be hard baked at temperatures above $120^{\circ} \mathrm{C}$, to crosslink and stabilize the remaining photoresist but is dependent on the application of the patterned resist. In this way, highly reproducible designs can be patterned onto substrates (129).

\subsubsection{Deposition and Thin Film Patterning}

Often MEMs applications require that additional material to be deposited and/or patterned on the devices being fabricated. These films may act as conducting, insulating, or adhesion layers and can be crystalline or amorphous. Typically, deposition is achieved through either chemical vapor deposition (CVD), or 
physical vapor deposition (PVD) (128). PVD is commonly used to create thin films of metals and is achieved by using a solid starting material (often referred to as a target) to create a vapor phase which is then deposited onto the substrate where it is converted back into a solid phase as a thin film (128). A number of different PVD techniques exist including electron beam deposition, thermal or evaporation deposition, and sputter deposition among others (128).

Sputter deposition entails creating a glow discharge plasma between two electrodes in a vacuum (139). To form a glow discharge plasma a partial pressure of an inert gas, such as argon, must generated in the chamber. Naturally, some fraction of this gas will be ionized by cosmic rays, such that when a voltage is applied to the chamber electrodes and an electric field is generated between them, the positively charged $\mathrm{Ar}+$ cations accelerate towards the cathode and negatively charged $\mathrm{e}^{-}$anions accelerate towards the anode (140). At sufficient voltage and chamber pressure the mean free path of the ions allows them to gain enough kinetic energy during their acceleration that they collide with other uncharged gas molecules in the chamber and ionize them to amplify the number of ions in the plasma (141). This cascade effect generates a plasma which can be sustained and used to sputter a desired target material, which is placed at the surface of the cathode. Ar+ cations colliding with the surface of the target at the cathode can knock atoms from the surface of the target which are then deposited on the substrate at the anode (140). While effective, glow discharge sputtering often has very low sputtering rates as the 
a)

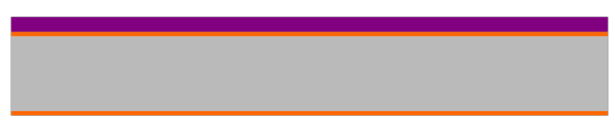

c)

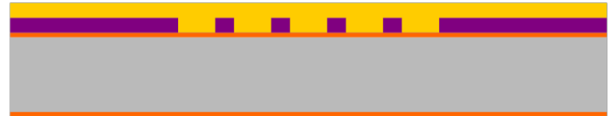

b)

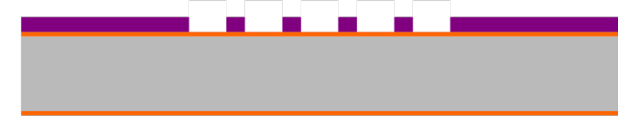

d)

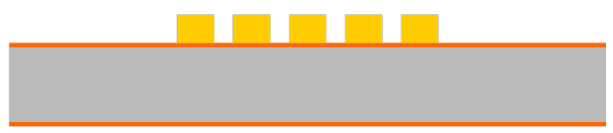

Figure 2.5: a) Lift-off deposition starts by coating a wafer (gray and orange) in photoresist (purple). b) The photoresist is patterned with the desired design. c) A thin film of the desired metal (yellow) is sputtered onto the wafer. d) The wafer is placed in an organic solvent to remove the photoresist and thin film layer on top of it, leaving behind the thin film in regions which did not have photoresist.

distance between the electrodes results in low plasma density at the surface of the cathode causing slow deposition rate (140). By placing magnets in the cathodes such that one pole is at the center of the cathode and the opposite pole is a ring surrounding the outside of the cathode, a magnetic field is generated parallel to the surface of the cathode (140). This magnetic field can trap electrons at the surface of the cathode and increases the plasma density to increase sputtering rate. In this way, films hundreds of nanometers thick with very small grains and low surface roughness can be created.

Patterning a thin film can be achieved through two different techniques known as lift-off deposition or etch-back deposition (129). In the latter, the thin film is first deposited on the wafer at the desired thickness. Then a sacrificial layer, such as a photoresist, is deposited on top of the deposited thin film and pattered using photolithography with the desired pattern. The wafer is subsequently etched with an etchant that targets only the thin film, where the sacrificial layer protects the 
thin film underneath it and the thin film is etched away wherever it is not protected by the patterned layer (129). After etching, the sacrificial layer is stripped leaving only the patterned thin film behind, shown in Figure 2.5a. Disadvantages of etch-back deposition include undercutting of the sacrificial layer due to the isotropic etching behavior of most liquid etchants, and depending on the thickness of the film this under etching can become quite significant and can affect the functionality of the final device depending on application. Lift-off deposition is effectively a reversal of etch-back, where the sacrificial layer, typically a photoresist, is coated on the surface of the wafer and patterned with the desired design (129). This is often done with a negative resist which results in either a vertical or inward tapered resist profile (142). The thin film is then deposited on top of the wafer to the desired thickness, where the negative profile of the resist disconnects the thin film deposited on the wafer from the thin film deposited on the resist surface. The resist is later removed with an organic solvent and the thin film deposited on the surface of the wafer is left behind, depicted in Figure 2.5b. Disadvantages of lift-off deposition are incomplete removal of the sacrificial resist, redeposition of thin film which has been lifted off, and so called "dog-ears" which occur when the deposited film leaves vertical structures which have deposited on the side walls of the sacrificial resist layer (128). 


\subsubsection{Etching}

Often microfabrication processes require that material is removed to form channels, or patterned features on the surface of the device. This can be done chemically with liquid etchants (known as wet etching) or physically with a plasma (known as dry etching). Wet etching is perhaps the most versatile, where a large number of liquid etchants have been described for various conductive and nonconductive materials (143). While wet etching of metal thin films is important for etch-back patterning, the etching of silicon especially important for microfabrication of both MEMs and semiconductors.

Two of the most common wet etchants used is the processing of silicon wafers are $\mathrm{HF}$ and potassium hydroxide $(\mathrm{KOH})$. $\mathrm{HF}$ is one of the few compounds that etches silicon oxide layers, which naturally form on the surface of silicon substrates under atmospheric conditions (144). Especially for electronics, the removal of oxide layers is important as it can also act as an insulator between features on the device (145). $\mathrm{KOH}$ is a commonly used etchant of silicon due to its anisotropic etching behavior, where the [111] plane of the crystalline silicon is etched at a considerably lower rate than the other planes (146). In a [100] oriented silicon substrates this anisotropic etching results in pyramidal shaped etching structures and depending on the orientation of the substrate other structures such as vertical channels can be formed with $\mathrm{KOH}$ etching. While a wide number of other wet etchants exist for various metals (143), describing each 
is beyond the scope of this introduction. The ones commented on have relevance to the work performed throughout the rest of this thesis.

Dry etching can also be used to etch substrates through a process known as reactive ion etching (RIE). A plasma is generated in the chamber, where the gas used for plasma formation is dependent on the substrate being etched (147). For example, sulfur hexafluoride $\left(\mathrm{SF}_{6}\right)$ is typically used for etching silicon, where the ions in the plasma are accelerated towards the substrate and etching occurs through sputtering or chemical etching. While useful, RIE etching of silicon is only typically on the order of microns deep and the aspect ratio of features etched is low (147). Deep reactive ion etching (DRIE) is a dry etching method that creates deep trenches is silicon with high aspect ratio side walls (148). This is achieved by alternating etching and passivating steps, where silicon is etched with an SF6 followed by a deposition step of $\mathrm{C}_{4} \mathrm{~F}_{8}$ which deposits as Teflon on the substrate (148). Teflon deposited on the vertical sidewalls during etching does not etch as fast as the Teflon at the bottom of the etching trench, so that the depth of the channel is increased at a significantly faster rate compared to the etching of the side walls (148). This process is known as the Bosch process (named for the company that discovered and patented the technique) and it can achieve features with depths of 200 um or greater with nearly vertical sidewalls.

\subsubsection{Wafer Bonding}

Vertical packaging of devices through wafer bonding is an important process for MEMs fabrication by creating hermetic sealing of chambers or channels. A 
number of different strategies can be used for bonding depending on the surface properties and identities of the films being bonded together (149-154). Direct, anodic, and eutectic bonding are three types of bonding which can be used to provide wafer level bonding. Each technique has different requirements for surface treatment and roughness of the films being bonded together, and each has advantages in the strength and utility of the bond being formed.

Direct bonding between films occurs when the atoms of adjacent wafers are brought into very close contact and elevated temperature (155). Covalent bonding forms between the molecules and adjacent films, resulting in strong, hermetic bonding between the two films $(128,156)$. The effectiveness of direct bonding is highly dependent on the surface roughness of the two films being bonded together, where a rough surface will not allow close enough proximity to form the covalent bonds necessary for bonding to occur (157). Plasma activation of silicon surfaces has been demonstrated to allow for direct bonding at lower temperatures (158), advantageous for processes which require low temperatures so as not to affect other structures on the wafers. However, the techniques sensitivity to surface roughness requires the ability to polish or obtain films with atomic level smoothness (159).

Anodic bonding is the process of bonding two wafers by creating a potential across the gap between the wafers resulting in their bonding. This is commonly done between silicon and glass, where the glass substrate is a borosilicate glass (such as pyrex) importantly containing sodium ions (160). When the wafers are 
brought into contact and a field is applied the sodium ions in the borosilicate glass diffuse towards the cathode and oxygen ions diffuse towards the anode and bond interphase (161). The diffusion of oxygen to the bond surface results in covalent bonding between the borosilicate glass and silicon wafer (162). While bonding between wafers sputter coated with thin borosilicate glass layers, electrical breakdown of the glass film with high enough potentials can cause bond failures $(163,164)$. While the technique is less sensitive to surface cleanliness than wafer bonding, voids in the film can still be formed by surface contaminants.

While two metal films can theoretically be heated to their melting point and pressed together to cause sealing, the temperatures required to reach the melting points of most of the films and substrates used in microfabrication make this method impractical. Eutectic systems are alloys where the combined melting point of two materials is considerably lower than the melting points of each individual material $(128,165)$. Examples of eutectic systems are gold-silicon, copper-tin, and aluminum-germanium (166). When films on adjacent wafers are brought into contact with high pressures and a temperature above the eutectic point of the two films, melting of the films occurs at their junction where the wafers can be effectively "welded" together (167). Eutectic bonding has less sensitivity to surface roughness but typically requires higher temperatures and pressures than anodic and direct bonding. 
The following chapters combine the techniques and strategies described here to create new instrumentation for use in conjunction with liquid cell transmission electron microscopy towards visualizing biological dynamics in a native hydrated environment. 


\section{Chapter 3: A Holistic Approach to Structural Biology - Characterization at the Atomic and Cellular Level}

\subsection{Introduction}

Understanding the structure-function relationships of biomolecules, macromolecular complexes, and even cellular ultrastructure is an important step towards harnessing biological machinery for industrial purposes or medical applications. TEM imaging of biological samples has been important in determining the structures of proteins (64), viruses (65), and whole cells (63). While a number of different structural techniques exist, from single particle analysis, to crystallography and whole cell tomography, ideally a holistic approach involving all approaches from single molecule to whole cell can be used to provide complete context of a system or pathway. This chapter describes atomic resolution characterization of a cyanobacterial protein and whole cell imaging and tomography for ultrastructure characterization.

\subsection{Cell Free Expression of Soluble Protein for 2D Crystallography}

The vast majority of known protein structures to date have been solved with $\mathrm{x}$-ray crystallography, although membrane proteins have proved challenging to crystalize and some proteins do not form large stable crystals required for the technique. 2D electron crystallography and single particle techniques using transmission electron microscopy have gained popularity for their ability to determine the structures of difficult to crystalize proteins. For any of these 
techniques, a large amount of purified protein is needed to generate the data needed to solve a structure. In vivo expression using $E$. coli is commonly used for producing large quantities of proteins from a plasmid, but is limited in posttranslational modifications and can only synthesize proteins which do not affect the viability of the organism $(168,169)$. Furthermore, the process is often time intensive and can take days to weeks for production (170). For structural biology, crystallization and structure determination is challenging and difficult process which requires large amounts of protein. As such there is a need for a high throughput, fast method for producing large quantities of protein for crystallization trials and data generation.

Cell-free protein expression effectively entails harvesting the intracellular machinery and adding a plasmid containing the gene to be expressed. While not a new technique, it has classically been difficult to produce bulk quantities of protein on the same scale as is possible with in vivo methods (171). Recent advances however have allowed for production of tens of micrograms of protein from a single reaction that allows for sufficient biomass for structural studies. While an advantage of cell-free expression is the extract used can be from the same system as the desired protein (prokaryotic, eukaryotic, insect, ect.), the development and commercialization of a wheat germ extract by Yaeta Endo has lowered the barrier of entry to the field considerably (172).

The general process for cell free expression using a wheat germ extract kit from Cell Free Sciences is outlined in Figure 3.1. The gene of interest is first cloned 


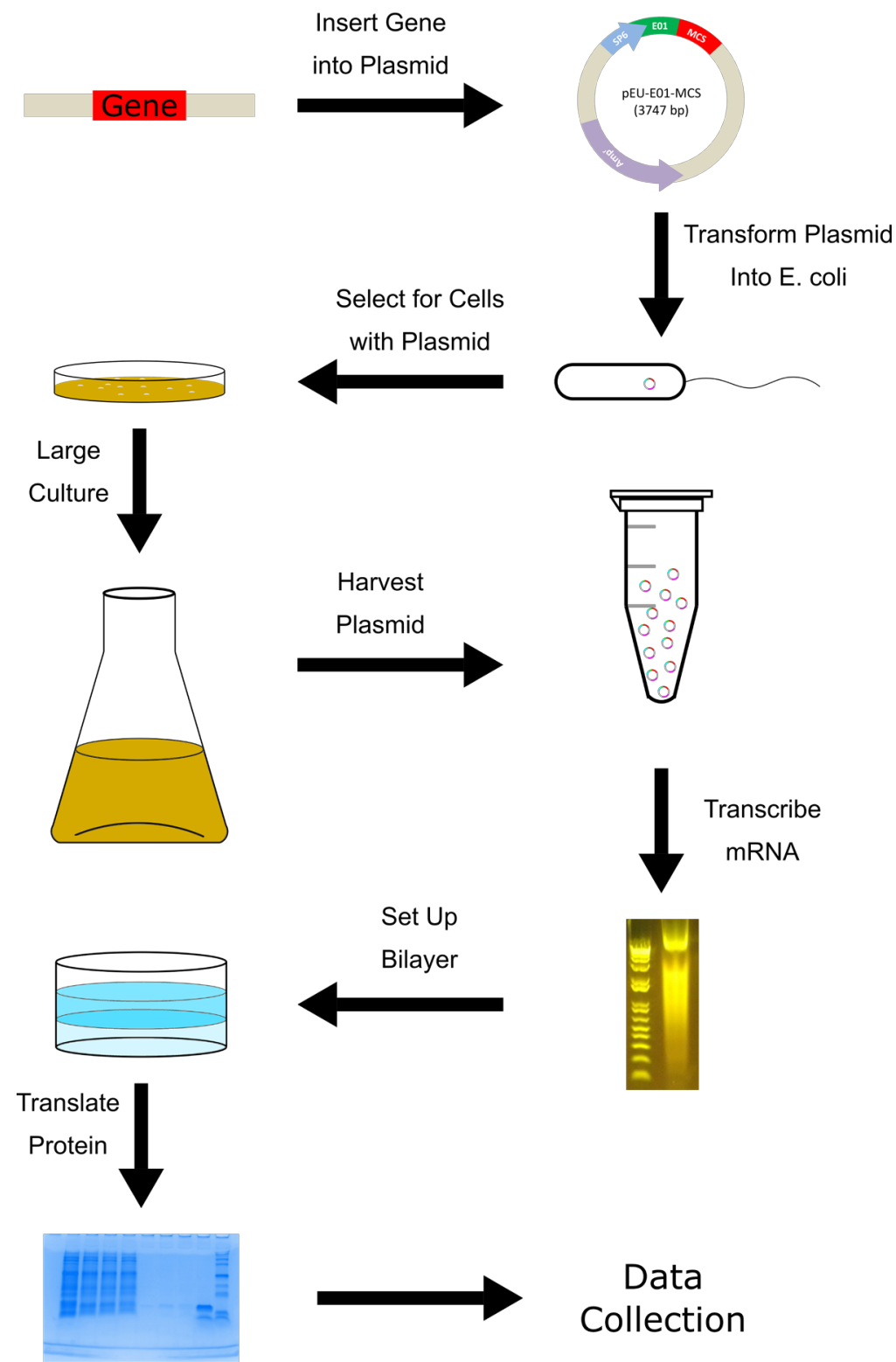

Figure 3.1: Cell free protein production entails inserting the gene of interest into an expression vector which is amplified using $E$. coli. Amplified plasmid is transcribed to form the mRNA template, which is transferred to the bottom of a bilayer reaction containing wheat germ extract. The produced protein is purified and can be used for a number of downstream applications.

into the $\mathrm{pEU}$ vector, which has been optimized for translation with wheat germ extract, using a Gibson assembly. During insertion, terminal His and FLAG tags are added to the protein sequence to aid in purification and crystallization. The plasmid is transformed into E. coli, successful transformant colonies are picked 
and cultured in a large volume to amplify the plasmid. The plasmid is then harvested from the cells and purified for use. Transcription is performed with the SP6 polymerase, and the resulting mRNA is combined with the wheat germ extract and deposited beneath a translation buffer in a bilayer reaction for protein production. After incubation the protein is harvested and purified using the added FLAG or His tags and purity is checked with SDS-PAGE. The final product is concentrated for use with structural studies and data collection. Both membranes and soluble proteins may be produced with this method as will be described below.

\subsubsection{Cell Free Expression of CcmK}

While classically defined as lacking distinct membrane bound organelles, many bacteria possess microcompartments with unique functions. Carboxysomes are protein microcompartments found in cyanobacteria and other chemoautotrophs which help to increase the rate of $\mathrm{CO}_{2}$ fixation by the RuBisCO reaction (173, 174). While they do not possess a lipid bilayer, the shell of the carboxysome is composed of small proteins which give it a faceted polyhedral structure (174). Often these structures are icosahedral but can be irregular as well (174). There are several different types of carboxysome shell proteins, where the organisms Synechocystis and Prochlorochocus produce shell proteins from the ccmk genes (173). A single monomer of the protein CcmK is $\sim 10 \mathrm{kDa}$ and known to be hexameric and it is thought the close packing of these proteins forms the faceted planes observed in carboxysomes $(173,175)$. The ordering of the proteins has 
allowed for 2D crystallization of the protein, where structures have been determined with electron crystallography $(176,177)$. This makes CcmK ideal to test the ability for protein produced via cell free expression to be crystalized and imaged with electron microscopy for structure determination.

The ccmk gene from Prochlorococcus marinus MIT 9313 was amplified and an $\mathrm{N}$-terminal 3X-FLAG tag and a C-terminal 10X-His tag were added to the gene. A second product was made with the $\mathrm{N}$-terminal 10X-His tag but without $\mathrm{C}$-terminal FLAG tag. The PCR product was then cloned into the Cell Free Sciences pEU vector using a Gibson assembly, amplified, and purified. The ccmk gene in the $\mathrm{pEU}$ vector is then expressed using SP6 polymerase to produce the mRNA transcript for translation. Because $\mathrm{CcmK}$ is not a membrane protein it can be synthesized directly with a bilayer transcription reaction. The translation mixture, consisting of wheat germ extract, creatine kinase, the mRNA transcription product is gently pipetted underneath a translation buffer in the well of a 24-well plate. The reaction was incubated at $15^{\circ} \mathrm{C}$ for $\sim 20$ hours before harvesting the protein product. CcmK is purified using magnetic beads functionalized with antiFLAG antibodies for FLAG purification or $\mathrm{Ni}^{+} \mathrm{NTA}$ for HIS purification. While FLAG purification typically gives better purity and yield, the presence of the FLAG tag on the C-terminus may potentially prevent crystallization. Figure $3.2 a$ shows an SDS-PAGE gel of the purity of the translation products through the steps of FLAG purification. The final concentrated product has good purity, where the second band is the excess FLAG that is used during the elution step. Figure 
a)

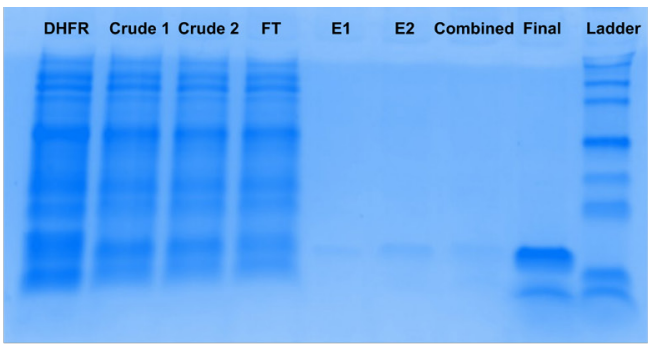

c)

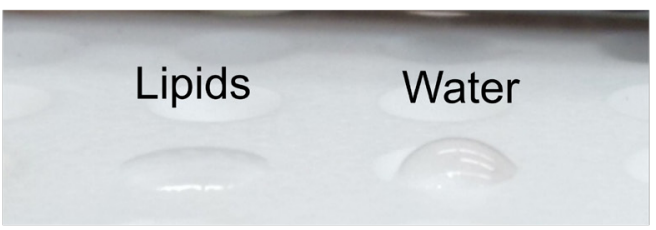

d)

e)

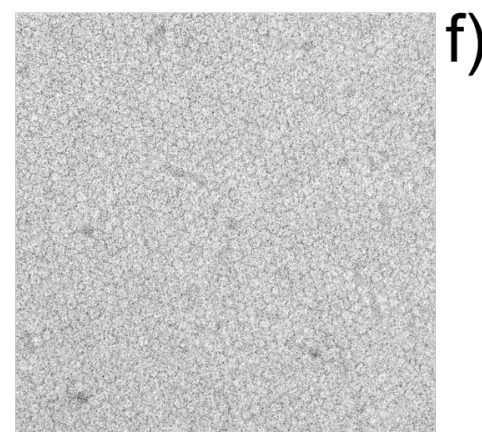

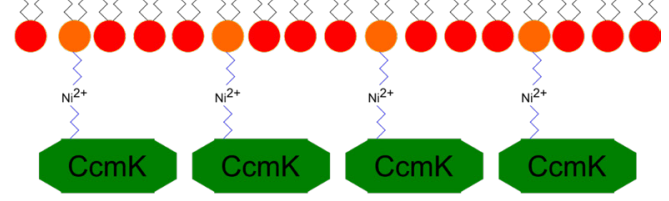

b)

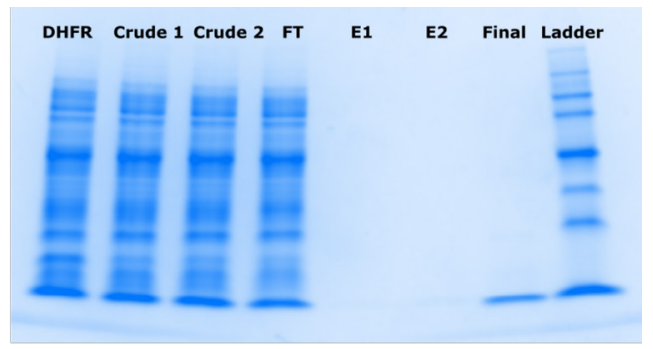

g)

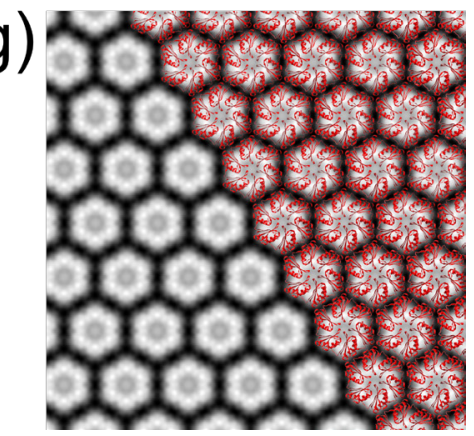

Figure 3.2: a) SDS-PAGE gel of CcmK during FLAG purification, bands in the final fraction have the CcmK protein and leftover FLAG from the elution fraction. b) SDS-PAGE gel of CcmK during His purification, the single band in the final fraction shows good purity. c) Image of the crystallization setup depicting how the addition of lipid monolayer reduces the surface tension of the aqueous buffer and allows for a flat crystallization surface. d) Illustration of the crystallization strategy where the lipid monolayer is doped with $\mathrm{Ni}^{+} \mathrm{NTA}$ groups which bind to the His-tagged $\mathrm{CcmK}$. e) Room temperature bright field STEM image of negative stained 2D CcmK crystals. f) Fourier transform of (e) after crystal unbending showing $13 \AA$ resolution reflections (orange arrow). g) Electron density map of CcmK crystals with the known x-ray structure overlayed on top of the map.

\section{2b shows an SDS-PAGE gel showing the purity of the CcmK protein}

throughout His purification. The concentration of imidazole in the wash buffer

was $50 \mathrm{mM}$, which appears to be enough to minimize non-specific binding

proteins but not too high to prevent binding of the His tagged CcmK. 


\subsubsection{D Crystallography of CcmK}

Protein crystallization through a lipid monolayer at the air-water interphase can aid crystallization of both soluble and membrane proteins which are otherwise difficult to crystalize with conventional techniques (178). The His tags engineered onto proteins for purification purposes may be leveraged for crystallization, where $\mathrm{Ni}^{2+} \mathrm{NTA}$ labeled lipids doped into a lipid monolayer can bind to His tags to promote associating between adjacent proteins $(175,177)$. An advantage of this technique is that it uses small volumes of concentrated protein. Figure 3.2c shows $15 \mathrm{uL}$ droplets of concentrated protein $(0.5 \mathrm{mg} / \mathrm{mL})$ in Teflon wells. The droplet on the left shows the decreased surface tension achieved after adding 2 uL of a lipid mixture of DOPC:DGS-Ni ${ }^{2+N T A}$ at a ratio of $4: 1$. The lipids form a monolayer at the water air interface with the hydrophobic tails on the air side and the polar head groups towards the liquid phase. Figure $3.2 \mathrm{~d}$ illustrates this crystallization strategy, where the His tagged $\mathrm{CcmK}$ in solution bound to the $\mathrm{Ni}^{2+} \mathrm{NTA}$ lipids in the monolayer. When the ionic strength of the buffer solution was correct, the $\mathrm{CcmK}$ proteins in solution orientated into a two-dimensional crystal on the surface of the lipid monolayer.

The crystallization of the proteins in solution can be slow, and often depends on the concentration of protein in solution. Incubation can take place from hours to days for complete crystallization to occur. In the case of $\mathrm{CcmK}$, the incubation required for 2D crystal formation was 13 hours to form large area crystals (hundreds to thousands of $\mathrm{nm}^{2}$ in area). After incubation the crystals are 
harvested with an electron microscopy grid with a carbon film. These films typically are natively hydrophobic and may be dropped onto the liquid droplet in the wells, where the hydrophobic tails of the lipids will bind to the hydrophobic carbon film on the EM grid. The sample was then frozen for cryo-EM studies or negatively stained with phosphotungstic acid for room temperature EM analysis.

Figure $3.2 \mathrm{e}$ shows a (contrast inverted) HAADF-STEM image of CcmK crystals grown using the method described above. It was found that proteins with the $\mathrm{N}$ terminal FLAG tag would not crystalize for any of the crystallization conditions which were attempted. We suspected that the FLAG tag prevented close packing of the protein to form a crystal. As a result, $\mathrm{CcmK}$ with only an $\mathrm{N}$-terminal His tag was used for crystallization. While it was expected that some non-specific binding proteins would not be removed during the purification step, these impurities were low enough to not significantly out-compete binding with $\mathrm{Ni}^{2+} \mathrm{NTA}$ groups on the lipid monolayer.

An advantage of 2D-electron crystallography is that the crystals formed can be poorly ordered and still give valuable structural information by using image processing $(179,180)$. While the real space image of the protein crystal is typically low contrast and has a high amount of noise, the power spectrum of the image shows the structural information in discrete spots from the periodicity of the crystal lattice (181). Fourier filtering can be used to remove excess noise from the image, at which point crystal defects and lattice distortions are observable (181). These distortions can be corrected through a lattice unbending 
process which uses cross correlation of a theoretical, or measured from a small subsection of the image, unit cell to calculate the shift vectors of the lattice distortions (181). These distortions can then be corrected for and, through iterations, as the lattice is refined the unit cell can be determined more accurately. The resulting power spectrum of the refined lattice results in diffraction spots which are more focused, and often reflections at higher resolutions than were apparent from the original image can be seen. These imaging processing tools have been compiled into a single tool for high throughput data generation in the 2DX package, which is now part of the Focus image processing suite (182-184). The power spectrum of the unbent and refined CcmK lattice from Figure 3.2e is shown in Figure 3.2f. The red arrow in Figure 3.2f highlights the highest resolution reflection at $13 \AA$, which is typically the resolution limit for negative stained samples. An advantage over x-ray and electron diffraction crystallography which give only amplitude information, is the power spectrum gives both amplitude and phase information (179). This information is used to create a final projection map of the protein crystal, shown in Figure 3.2g. The three-dimensional structure of the protein crystal can also be determined if images are collected from multiple tilt angles, where the resulting projections can be combined to determine the three-dimensional structure of the protein. While only a single 0 -tilt projection was collected for this CcmK crystal, the measured lattice agrees closely with the known x-ray structure of the protein, demonstrating that cell-free expression can be used to produce proteins for structural determination through 2D crystallography. While further work to obtain 
higher resolution structures could have been performed, this was unnecessary for $\mathrm{CcmK}$ as the structure of the protein had already been determined at high resolution with $\mathrm{x}$-ray crystallography. The purpose of this experiment was to demonstrate that protein produced with cell free expression could be successfully crystallized and the resulting protein structure aligned with existing structures. With confidence that the pipeline is capable of producing large quantities of proteins with proper folding and post translational modifications structural studies can begin on proteins with unknown structure.

\subsection{Cell Free Expression of Membrane Proteins for Single Particle Analysis}

Membrane proteins are notoriously difficult to perform structural studies due to their insolubility in water without stabilization with detergents. Isolation, purification, and crystallization of membrane proteins produced in-vivo from organisms is a non-trivial task where the throughput is often reduced due to the individual approach required for each membrane protein. Cell free expression allows for direct synthesis of the desired membrane protein into a membrane for stabilization, where purification to separate from other membrane proteins is not necessary. As such, it represents an ideal platform to create biomass for structural studies with electron microscopy.

Triacylglycerols are a class of lipids which have potential as biofuel feedstocks and are known to be produced by algae under nutrient limiting growth conditions (185). However, their metabolic pathway is not well understood including the final 
enzyme diacylglycerol acyltransferase (DGAT) which is membrane bound and thought to localize to the membrane of the endoplasmic reticulum (186). Towards the goal of industrial engineering of algae for biofuel production, the synthesis of triacylglycerols and the function of DGAT needs to be better understood, and its structure is currently unknown. The ability to produce protein with cell free expression for crystallization and structural studies is a critical step in characterizing the triacylglycerol synthesis pathway.

\subsubsection{Cell Free Expression of DGAT into Liposomes}

In O. tauri, the gene dgat consists of two exons of length $0.2 \mathrm{kB}$ and $1.2 \mathrm{kB}$. The exons were cloned from genomic DNA from the microalgae $O$. tauri using polymerase chain reaction (PCR). The exons were amplified separately to remove the single intron, where the exon PCR products are shown in Figure 3.3a. During PCR the N-terminus was modified with a 3X-FLAG tag and the Cterminus was modified with a 10X-His tag. The amplified exon fragments were gel purified and transferred to the pEU plasmid using a Gibson assembly. The resulting plasmid was transformed into $E$. coli (NEB-10) and streaked onto an agar plate with ampicillin for selection of successfully transformed cells. Resulting colonies were picked and sent for sequencing to verify integrity of the plasmid insert. Successful clones were grown in a large liquid culture to amplify the plasmid, and after growth the plasmid was harvested and purified.

The DGAT gene was transcribed with an SP6 polymerase, and the resulting mRNA produced was combined with wheat germ extract and liposomes and 
a)

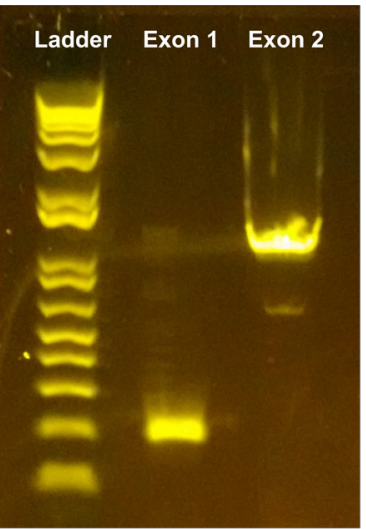

b)

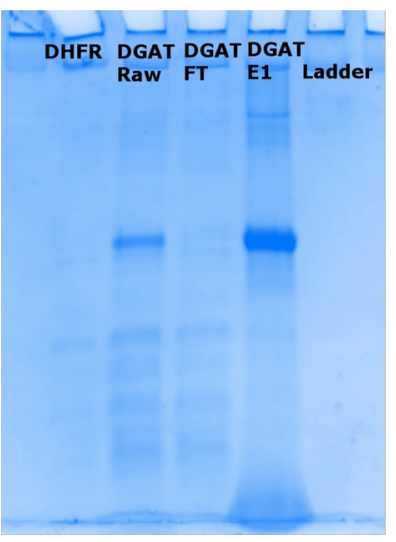

c)

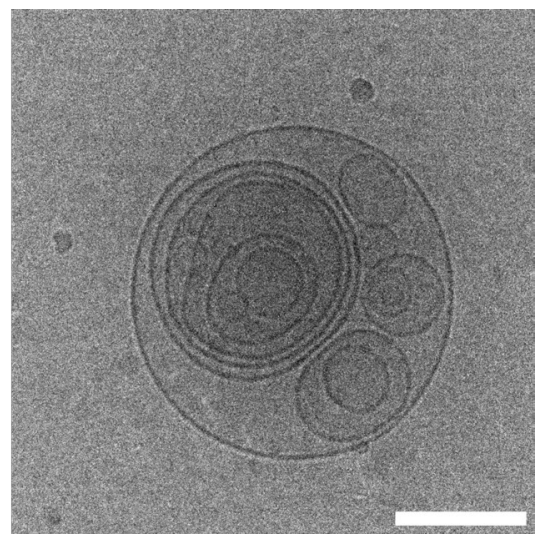

Figure 3.3: a) Agarose gel showing exons 1 and 2 of dgat. b) SDS-PAGE gel of DGAT from cell free expression and purified with FLAG purification. c) Bright field transmission electron micrograph of liposomes containing DGAT. Scale bar is $100 \mathrm{~nm}$.

placed below a transcription buffer to set up a bilayer reaction. As the mRNA is translated the membrane portion of DGAT is synthesized into the included liposomes. After incubation overnight, the liposomes with integral membrane proteins (proteoliposomes) can be harvested simply by centrifugation and washing to remove unwanted soluble proteins. Figure 3.3b shows an SDS-PAGE gel of the products showing a final product with good purity.

After expression, the liposomes were imaged with cryo-EM to observe the size and morphology of the produced liposomes. Figure $3.3 \mathrm{c}$ shows a cryo-EM image of a liposome which has copies of DGAT within the layers. As is seen, the formed liposomes are multilamellar. For cryo-EM single particle studies ideally these structures will be unilamellar $100-200 \mathrm{~nm}$ liposomes. Protein is not clearly visible in the liposome membranes in the image in Figure $3.3 \mathrm{c}$, as the $50 \mathrm{kDa}$ protein is not large enough to be visible without staining on the CCD the image was taken with. Ongoing work is to use a direct electron detector and phase plate to obtain 
images of DGAT with sufficient contrast to perform single particle studies. Additionally, crystallization trials will be run to form 2D crystals of DGAT for 2D crystallography.

\subsection{Correlating Loss of Structural Information Under Electron Irradiation Using 3D Protein Crystals}

Catalase is a well described protein and is easily crystalized into high aspect ratio sheets which can by one or several layers in thickness making them ideal for electron microscopy. In particular, they have classically been used to characterize electron irradiation damage (187). Furthermore, catalases well documented crystallization and availability make it an ideal protein for studying the effects of electron irradiation induced damage. Because of the well documented behavior of resolution loss of catalase in cryo-EM (187-192), it was an ideal sample to try and study resolution loss in LC-TEM studies, described later in section 5.6.3. As such to verify our ability generate quality crystals, preliminary data was collected from cryo-EM damage series on $2 \mathrm{D}$ catalase crystals grown in-house. While a number of different methods of catalase crystallization are known, the technique described by Dorset and Parsons was used to crystalize bovine liver catalase purchased from Sigma Aldrich (188). Often, purified catalase will already be assembled into several different types of crystals depending on the $\mathrm{pH}$ of the solution, often in the shape of needles. The purchased stock solution was solubilized at $4^{\circ} \mathrm{C}$ in $\mathrm{pH} 7.4 \mathrm{KH}_{2} \mathrm{PO}_{4} / \mathrm{Na}_{2} \mathrm{HPO}_{4}$ buffer with constant gentle shaking for 12 hours. After removing insoluble 


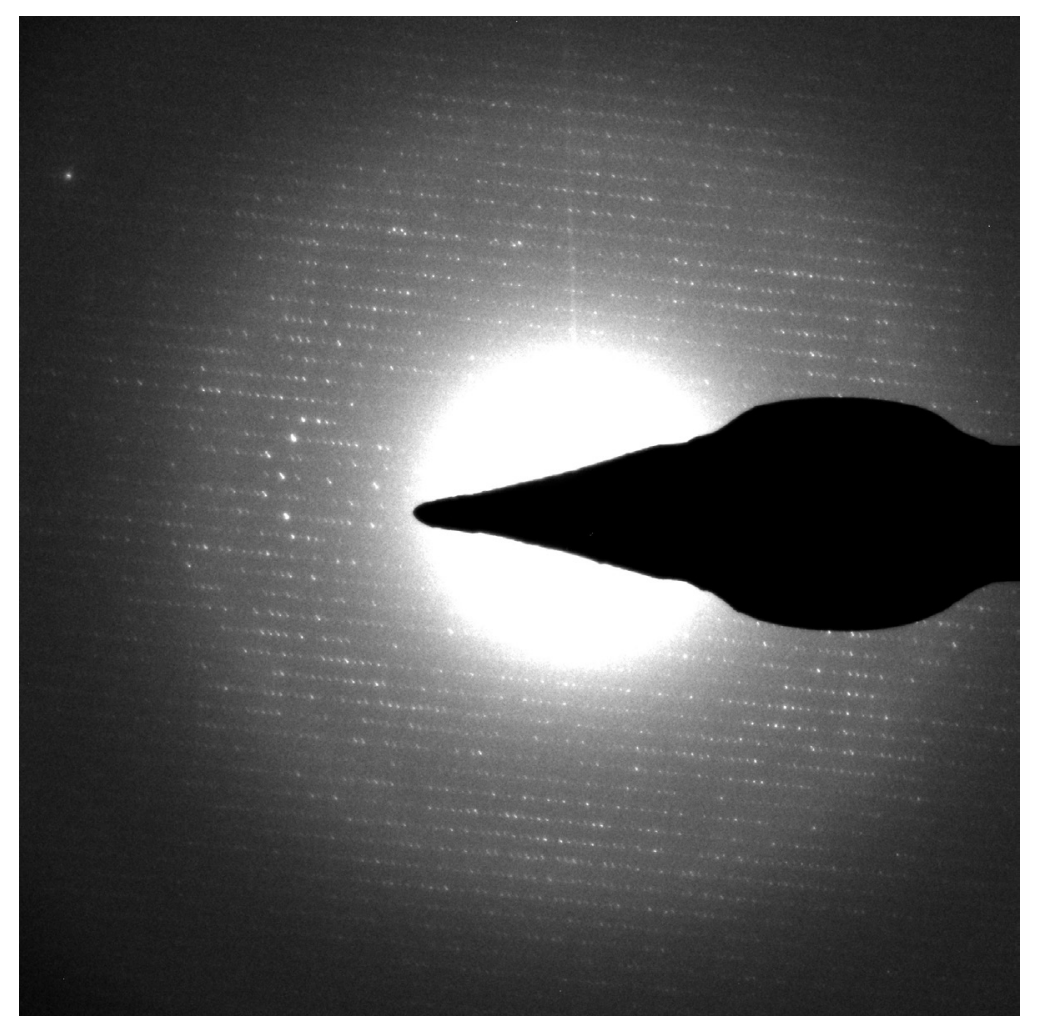

Figure 3.4: Electron diffraction pattern of 3D catalase crystals. Outermost reflections visible are 2$3 \AA$ resolution.

products by centrifugation, the solubilized protein was titrated to $\mathrm{pH} 5.3$ with a saturated $\mathrm{KH}_{2} \mathrm{PO}_{4}$ solution and incubated at $4^{\circ} \mathrm{C}$. After several days crystals had precipitated from solution and could be harvested for electron microscopy experiments.

Catalase crystals grown with this technique form large 2D sheets which can be microns in length and width. While often seen as a single sheet, they can also stack forming sheets several protein layers in thickness creating 3D crystals. Figure 3.4 shows a high resolution electron diffraction pattern of catalase crystals taken with cryo-EM. The outermost reflections in this image are 2-3 $\AA$ resolution 


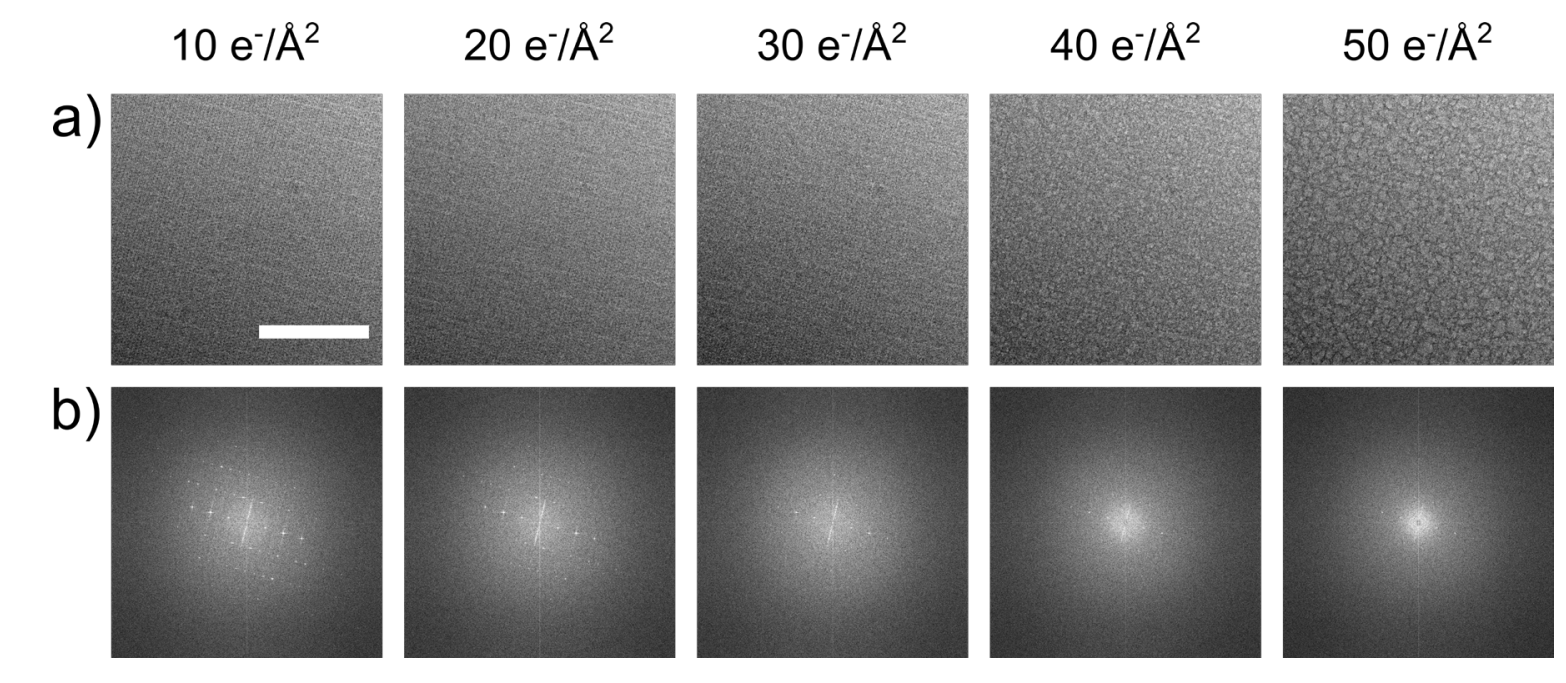

Figure 3.5: a) Bright field TEM images of catalase crystals for sequentially increasing cumulative electron flux. Damage in the form of bubble formation is visually apparent by $50 \mathrm{e}^{-} / \AA^{2}$. b) Fast Fourier transforms corresponding to each image in (a). Reflections indicating crystallinity are lost with increasing cumulative electron flux. Scale bar in (a) is $100 \mathrm{~nm}$.

diffraction spots, demonstrating our ability to obtain atomic resolution images of 3D protein crystals.

\subsubsection{Damage of Catalase with Electron Irradiation}

Several damage experiments were performed on catalase crystals frozen in vitrified ice with cryo-EM, and damage data was obtained for both real space and diffraction images. Images were acquired by exposing the catalase crystals to an electron flux of $10 \mathrm{e}^{-} / \AA^{2}$ and repeated 5 times until obvious bubble formation was seen, or diffraction reflections were no longer observed. Figure 3.5a shows real space images of catalase crystals in ice at increasing cumulative electron fluxes. Towards the end of the imaging series bubble formation can be seen, which is typical for biological samples imaged with cryo-EM as a result of the liberation and buildup of hydrogen gas from radiolysis (193). The power spectrums of these 
images are shown in Figure 3.5b, where higher order reflections are lost as a function of increasing cumulative electron flux. The irradiation sensitivity and loss of resolution matches with what has been described in literature for damage thresholds of catalase crystals. These crystals were then used for LC-TEM experiments in an attempt to characterize how electron damage in liquids compares to damage in cryo-EM as discussed in chapter 5.6.3.

\subsection{Whole Cell Imaging}

While structural studies on proteins often achieve atomic level resolution and are extremely valuable towards understanding the structure-function relationship of a protein, single particle and crystallography studies lack the context of the protein within a whole cell context. Furthermore, ultrastructural changes that cells undergo as a result of environmental stimulus or genetic modification are not apparent when only observing a single protein. Therefore, it is important to characterize whole cell physiologies and morphologies in conjunction with high resolution studies of molecules of interest. Several techniques can be used to image cells at high resolutions with TEM, where the high vacuum environment of the instrument and the damaging properties of the electron beam must be considered. The intrinsic low contrast of biological material due to their weak interaction with the electron beam is another limitation. The most simple form of sample preparation is negatively staining cells with a heavy metal salt, such as phosphotungstic acid or uranyl acetate, and then drying on a carbon TEM grid (36). Another strategy is fixing cells followed by embedding them in a resin, 

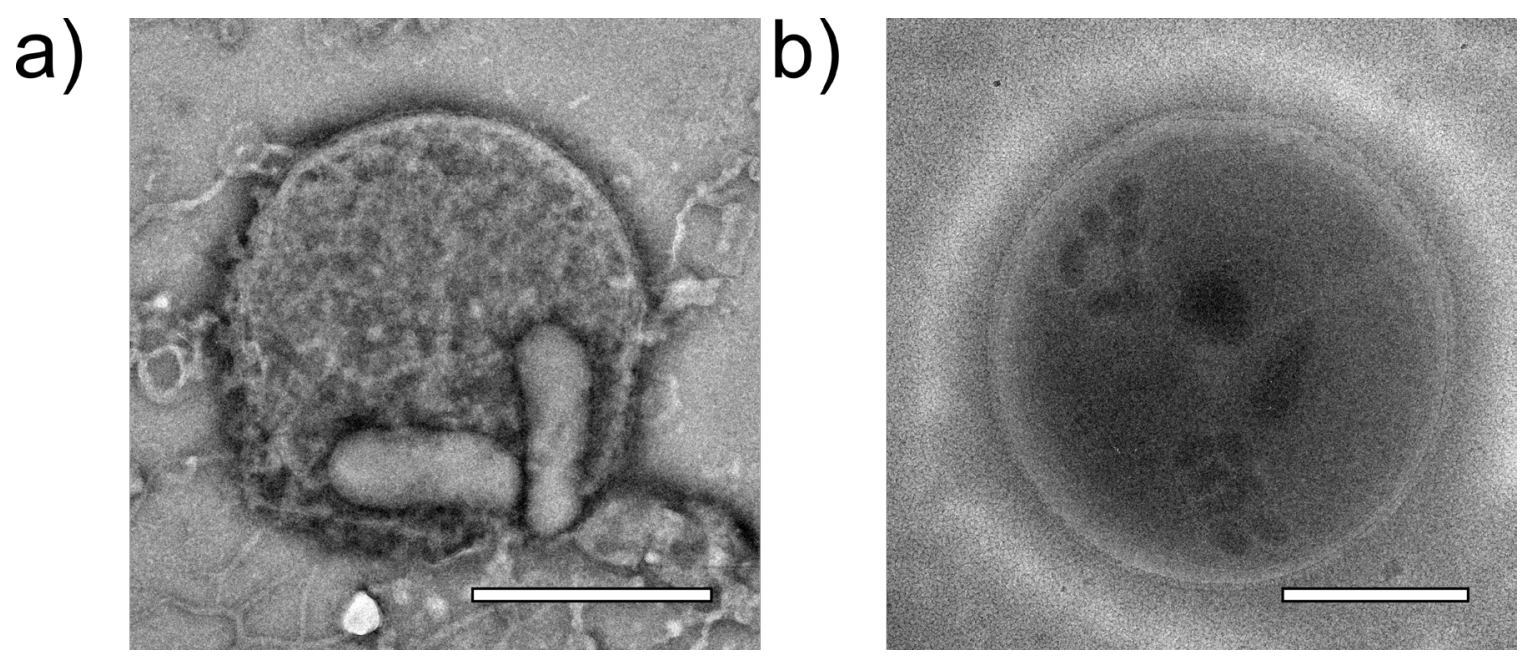

Figure 3.6: a) Negative stained, room temperature BF-TEM image of $O$. tauri. b) Cryogenic BF-TEM image of $O$. tauri illustrating increased detail compared to the negative stain, room temperature image in (a). All scale bars are $1 \mu \mathrm{m}$.

which can then be sectioned into thin slices for TEM imaging using ultramicrotomy (36). However, both of these conventional approaches can introduce artifacts in the samples, confounding image interpretation and analysis (194). Another method, cryogenic electron microscopy (cryo-EM), permits cells to be frozen in a layer of vitrified ice and kept at cryogenic temperatures preserving the native cellular context and composition (195-197).

\subsubsection{Negative Staining and Room Temperature Imaging}

Figure 3.6a shows an image of $O$. tauri which has been imaged with BF-TEM after negative staining. Sample preparation for this strategy is the simplest and fastest of all the techniques, where cells are deposited onto a glow discharged EM grid, negative stained for $\sim 60$ s (in phosphotungstic acid in this case), and blot dried. When placed into the microscope, any remaining liquid or volatile organics evaporate into the column in the high vacuum environment of the microscope. 
Only the "mold" remaining from the stain is imaged, where most of the biological material sublimates into the column or is destroyed by the electron beam. As a result, much of the structure of the cell is lost as membrane integrity and the structure of many proteins are dependent on hydration. Additionally, the metal stain cannot fully penetrate the crevices of all biomaterial, limiting the resolution of the technique. Negative staining gives strong contrast which is advantageous for imaging biological samples, although the remaining organic material is often still highly beam sensitive. As a result, damage of the remaining cellular structure is commonly observed which further degrades image resolution and drives the sample further from its normal physiological state. Comparatively, Figure 3.6b shows an image of $O$. tauri taken with cryo-EM imaging, showing a considerable increase in structural detail and cell integrity. While negative stained dry state TEM of whole cells does not allow for high resolution detail to be obtained, its quick sample preparation makes it well suited for sample screening as a precursor to performing more intensive cryo-EM studies.

\subsubsection{Cryogenic Electron Microscopy}

In order to counter the dehydration and sublimation of biological material of the cell when exposed to the high vacuum of the TEM, the sample can be frozen in a layer of vitreous ice and maintained at cryogenic temperatures. This not only helps maintain the structural integrity and hydration of the cell but also can slow the propagation of damage by constraining damage products from radiolysis within the frozen matrix (198). As seen by the comparison of dry state to cryo-EM 
images of the same cell in Figure 3.6, cryogenic preservation allows for substantially more subcellular detail. Figure 3.7 shows other cryo-EM images for whole cells which have been imaged with BF-TEM. Figure 3.7a is an image of the marine cyanobacteria Prochlorococcus marinus, the organism from which the CcmK protein in section 3.3.1 was taken from. Although this sample did not have the carboxysomes that are typical in many cyanobacteria (199), the multiple membranes of the organism is clearly visible in the images obtained. These multiple membranes are a unique feature to cyanobacteria which use them to increase surface area for the membrane bound light harvesting phycobilisomes to maximize photosynthetic activity.

Figure $3.7 \mathrm{~b}$ is an image of the microalgae Ostreococcus tauri, similar to the image shown in 3.6b. While one of the largest cells imaged in Figure 7, it is one of the smallest known eukaryotes with distinct membrane bound organelles. The ultrastructure (200) and morphological changes of $O$. tauri are of interest as it is known to produce lipid bodies of triacylglycerols during growth in nitrogen limited 
a)

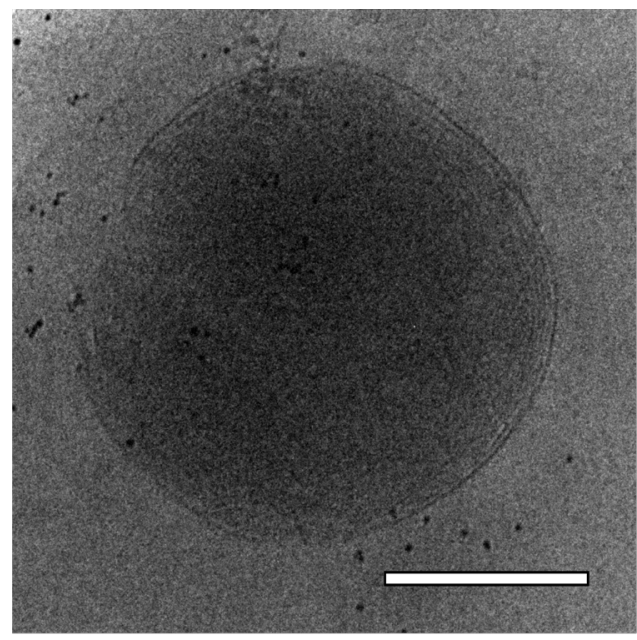

c)

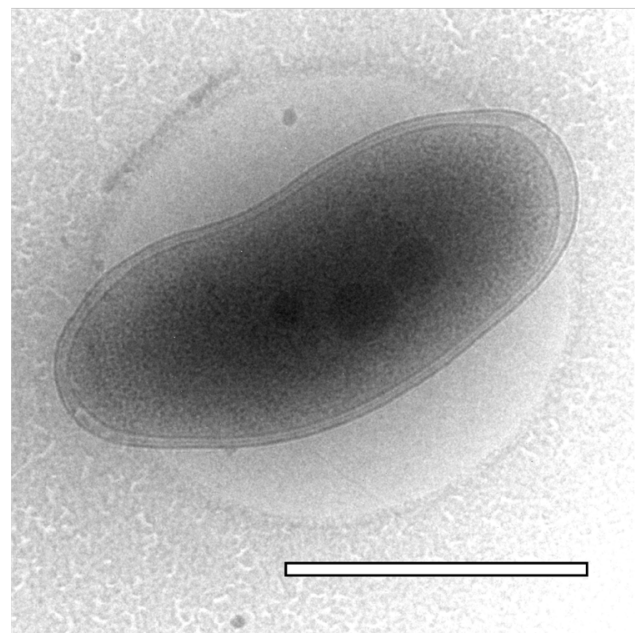

b)

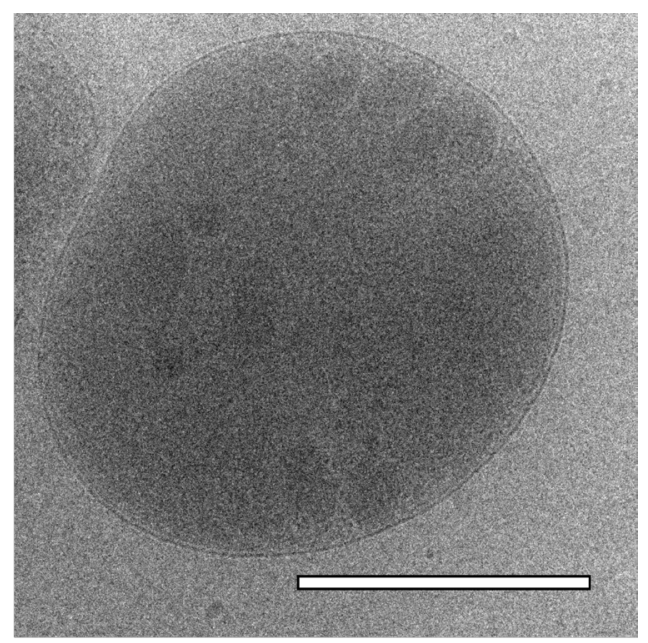

d)

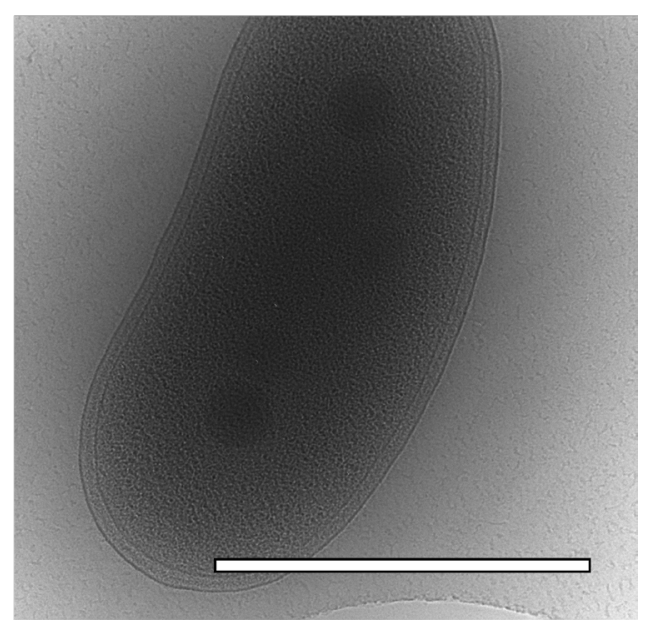

Figure 3.7: a) Phase contrast cryo-EM image of P. marinus. b) Phase contrast cryo-EM image of $O$. tauri. c) Phase contrast cryo-EM image of C. metallidurans. d) Phase contrast cryo-EM image of $C$. metallidurans taken with a phase plate and collected with a direct electron detector demonstrating improved contrast and signal to noise with these instruments when compared to (c). Scale bars are $500 \mathrm{~nm}$, $1 \mu \mathrm{m}, 500 \mathrm{~nm}$, and $500 \mathrm{~nm}$ respectively.

media $(201,202)$. The triacylglycerols that are synthesized and stored in these lipid bodies originate from the diacylglycerol acyltransferase described in section 3.2.1, where whole cell cryo-EM imaging and tomography was planned to provide ultrastructural context in coordination with atomic level information determined from the purified protein. While the generation of lipid bodies could 
be easily stimulated by limiting the growth media of nitrogen, whole cell imaging of $O$. tauri proved difficult, as cells imaged in cryo-EM were commonly ruptured after freezing. While other experiments freezing $O$. tauri in bulk solutions for imaging with $\mathrm{x}$-ray tomography and FIB-SEM imaging, the process of blot drying samples to achieve thin ice layers for cryo-TEM imaging seems to result in the enough surface tension on the cells to rupture them.

Figure 3.7c shows a cryo-EM image of the soil bacterium Cupriavidus metallidurans that is known for its high heavy metal resistance imparted by a number of genes which code for the RND class proteins for sensing and pumping several types of heavy metal ions (203-205). It is also known for its remarkable ability to biomineralize zero valence gold nanoparticles from aqueous environmental gold (206-208). While the exact mechanism is not clear, it demonstrates remarkable metabolic pathways with unique redox and oxidation capabilities. Understanding these pathways is of interest towards custom engineering of organisms with unique metabolic properties for biofuel production. Figure $3.7 \mathrm{c}$ is shown in contrast to Figure $3.7 \mathrm{~d}$, which is also an image of $C$. metallidurans but taken with state of the art detectors and contrast amplifying instrumentation. The image in Figure 3.7c is acquired on an image corrected 300 $k V$ TEM with a $2 k \times 2 k C C D$ at an electron flux of $10 e^{-} / \AA^{2}$. In comparison, Figure $3.7 \mathrm{~d}$ is an image of the same organism but acquired on a $300 \mathrm{kV}$ TEM with a phase plate, energy filter, and direct electron detector at an electron flux of $1 \mathrm{e}^{-}$ 
$\mid \AA^{2}$. The increase in contrast is noticeable, demonstrating the importance of instrumentation for acquiring ideal images.

\subsubsection{Cryogenic Electron Tomography}

In addition to generating 2D projection images, cryo-EM imaging on whole cell structures can be taken at multiple tilt angles on the same sample to obtain tomograms. The resulting tilt series can be reconstructed to form a 3-dimensional model of the sample imaged. Figure 3.8a-c shows the extreme tilts and no tilt image of a tilt series of $O$. tauri, where the images shown are the $-66^{\circ}$ tilt (a), the $0^{\circ}(\mathrm{b})$, and the $+66^{\circ}$ tilt (c) with images acquired every 3 degrees in between those points. The images obtained can then be finely aligned and back weighted projection is used to generate a z-stack of the resulting tomogram. Figure $3.8 \mathrm{~d}$ shows the center slice of a z-stack from the tilt series shown in Figure 8a-c. The features visible in the tomogram can be outlined at every z position to create a 3dimensional model of the cell. Figure $3.8 \mathrm{f}$ shows the resulting model, where individual organelles have been modeled. Of note, only a central section of the cell has been modeled, where the top and the bottom of the cell is missing. This is the result of the so called "missing wedge" that occurs in tomography due to the missing tilt angles at very high tilts which cannot be imaged due to the increasing thickness of the sample. As stated previously, extensive tomography of $O$. tauri was limited by the ability to capture images of cells which were not destroyed during the freezing process, and future work is focused on reducing 

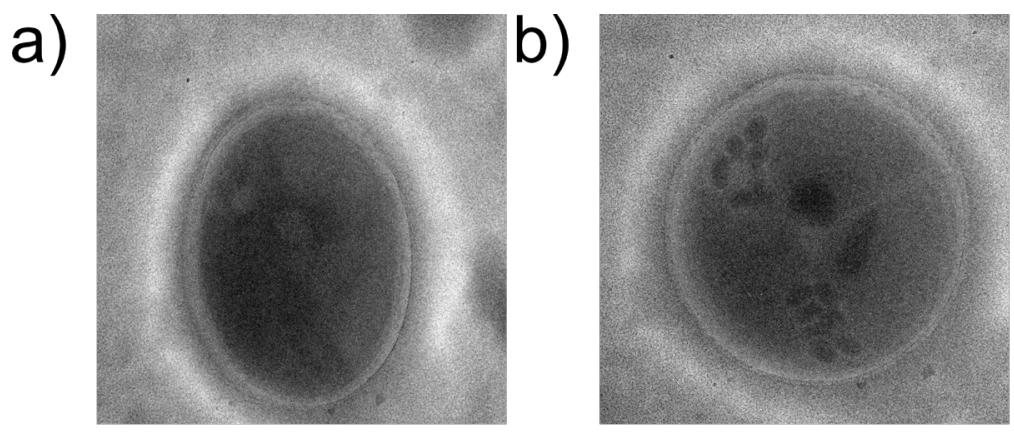

d)

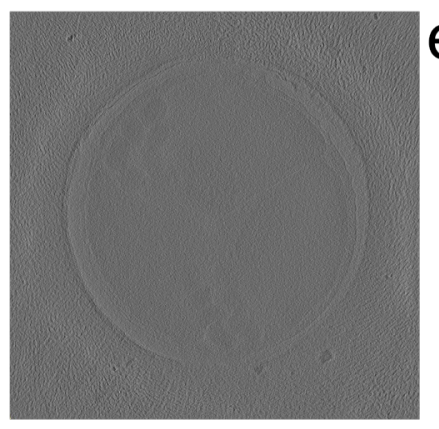

e)

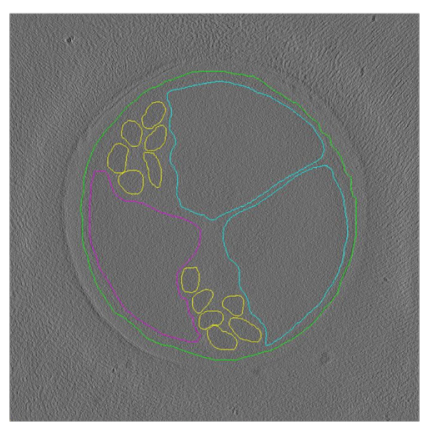

c)

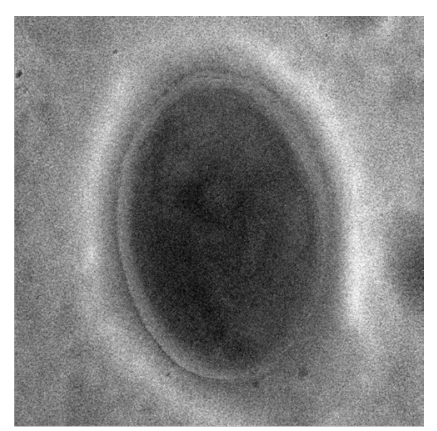

f)

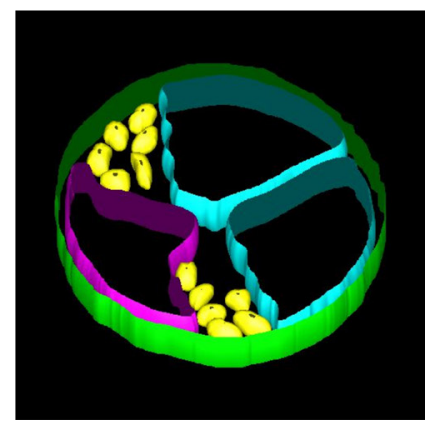

Figure 3.8: a) $O$. tauri at minus 66-degree tilt. b) $O$. tauri at zero-degree tilt. c) $O$. tauri at plus 66-degree tilt. d) Central slice from the resulting tomogram. e) Segmentation of subcellular structures in one slice of the tomogram. f) Resulting three-dimensional model of $O$. tauri.

the lysing of cells so that tomograms may be acquired of cells during different nutrient growth conditions to observe the formation and evolution of lipid bodies.

The methodology outlined in this chapter presents the groundwork towards characterizing individual molecules at atomic resolutions and within an "in-situ" context within a whole cell. By determining high resolutions of protein structures through cell free expression of proteins for single particle analysis and 2D electron crystallography, followed by whole cell imaging and tomography, the context of these biomolecules can be visualized within their native state and organization within the cell. Future work is focused towards labeling biomolecules and proteins of interest for targeting with subtomogram averaging which will give improved context of cellular organization and structure. 


\section{Chapter 4: Fabrication of Free-Standing Silicon Nitride Membranes for Liquid Cell Transmission Electron Microscopy}

\subsection{Introduction}

The reliable fabrication of thin, free standing membranes has been an important capability in the MEMs field for applications ranging from pressure sensors (152) to optical filters (153). Silicon nitride ( $\mathrm{SiN})$ is commonly used as a membrane material due to advantageous physical properties including corrosion resistance, thermal and mechanical stability, and optical absorption qualities. Thin films of SiN are most commonly formed using chemical vapor deposition techniques, where Si-N stoichiometry and film stress can be tuned based on application. The formation of free standing membranes can be achieved by etching through a silicon substrate using concentrated potassium hydroxide $(\mathrm{KOH})$, where the silicon nitride acts as both the etch stop and etch mask for the $\mathrm{KOH}$. Details for producing silicon devices with these free standing membranes which fit commercially available LC-TEM holders is described below, along with a demonstration of their scientific utility to understand electron-water interactions.

\subsection{Design of Nanofluidic Holders and Devices}

Replication of commercially available devices for use with commercial LC-TEM holders is constrained primarily by the geometry of the holder, including critically the dimensions of the o-rings used to hermetically seal the liquid sample, the aperture size for beam transmission, and the dimensions of the well which the 
a)

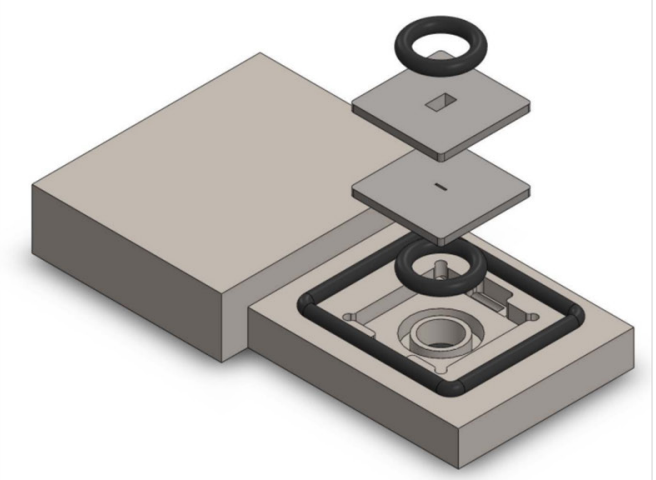

b)

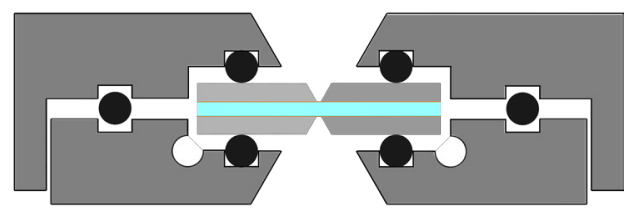

Figure 4.1: a) Illustration of an exploded view of the typical 3 o-ring sealing strategy for LC-TEM holders. b) Cross sectional illustration of the same holder from (a) demonstrating the sealing strategy. The liquid thickness between the nanofluidic devices is not to scale.

devices sit in. There are currently three commercially available holders which utilize free standing silicon nitride membranes supported by a silicon substrate to create a hermetically sealed chamber for imaging of liquid samples. While all three differ slightly in exact dimensions and design, each uses a series of o-rings to seal the liquid sample between two nanofluidic devices. A generic three o-ring design is depicted in Figure 4.1, where 4.1a depicts an exploded view of the nanofluidic devices and o-rings used for sealing. Of note, a lid is also used to compress the o-rings and devices together to achieve sealing and is not shown in Figure 4.1. A cross section of a fully assembled and sealed liquid cell is depicted in Figure 4.1b, showing the liquid sample sandwiched between the two nanofluidic devices. The critical dimension for nanofluidic device design is limited by the small o-rings, where the etched region forming the free-standing silicon nitride membrane must be within the inner diameter of the small o-ring for proper sealing. While the inner diameter of the smallest o-ring constrains the design for sealing, the imaging region is further constrained by the size of the aperture in 
the tip and lid which allows for transmittance of the electron beam through the sample. Imaging regions of the nanofluidic devices must fit within the constraints of this aperture to avoid the beam being blocked by the lid or tip itself.

\subsubsection{Devices for the Hummingbird LC-TEM Holder}

The dimensions for the upper and lower devices for the Hummingbird LC-TEM holders are identical as shown in Figure 4.2b. The length and width dimensions of the fabricated devices are approximately $2.6 \mathrm{~mm} \times 2.6 \mathrm{~mm}$, resulting in flexible orientation of the devices relative to one another during assembly. The depth of the holder well is $400 \mu \mathrm{m}$, so that each individual device has a thickness of 200 $\mu \mathrm{m}$. To achieve hermetic sealing of the liquid sample the Hummingbird holder uses three o-rings, a large outer o-ring with an inner diameter of $3.91 \mathrm{~mm}$ and a cross section of $0.32 \mathrm{~mm}$ with two smaller identically sized inner o-rings with an inner diameter of $1.24 \mathrm{~mm}$ and a cross section of $0.34 \mathrm{~mm}$. The outer o-ring does not have a constraint on the design of the devices as it is responsible for creating

a seal between the holder lid and tip depicted in Figure 4.1b. The inner o-rings seal directly onto the upper and lower device and constrain the location, shape, and size of the free-standing membrane region which can be formed while still maintaining ability for proper sealing. The limiting dimensions for the freestanding membranes then is the inner diameter of these o-rings and is depicted by the green circle in Figure 4.2a. While sealing is constrained by the inner o-ring dimensions the practical viewing area is limited by the smallest aperture in the 


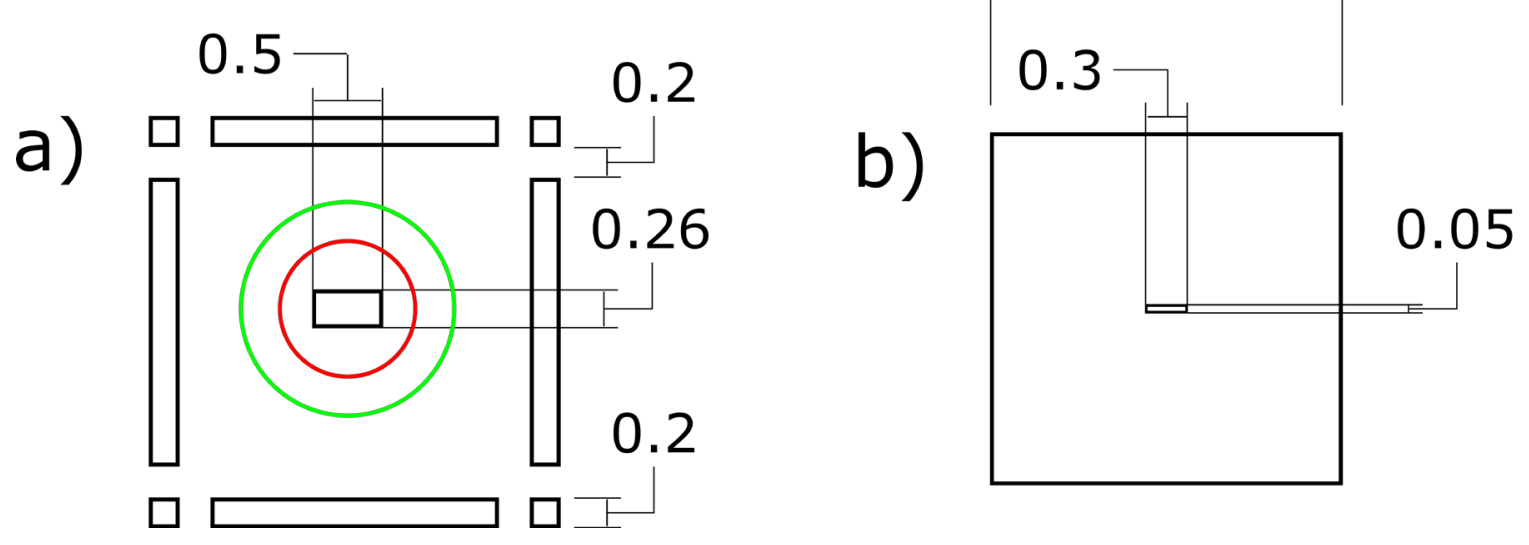

Figure 4.2: a) Mask design for devices which fit the Hummingbird Liquid Stage holder. b) Illustration of devices produced using the mask depicted in (a).

holder through which the electron beam passes. The smallest of these apertures on the Hummingbird holder is $0.79 \mathrm{~mm}$ in diameter, shown as a red circle in Figure 4.2a. Figure $4.2 \mathrm{a}$ additionally annotates the dimensions of the photomask features used to create the pattern for the devices during fabrication. Figure $4.2 \mathrm{~b}$ then shows the corresponding device that is formed using the mask dimensions from Figure 4.2a using a conventional fabrication protocol and results in a single free-standing membrane approximately $300 \mu \mathrm{m}$ in length and $50 \mu \mathrm{m}$ in width.

\subsubsection{Devices for the Protochips LC-TEM Holder}

The Protochips Liquid Stage holder uses a different sealing strategy from the Hummingbird and DENS Solutions holders utilizing two o-rings instead of three. Sealing is achieved by increasing the size of the upper device such that a larger o-ring seals against the underside of the upper device. The bottom device then seals against the holder against a small o-ring on the back side of the bottom 
a)

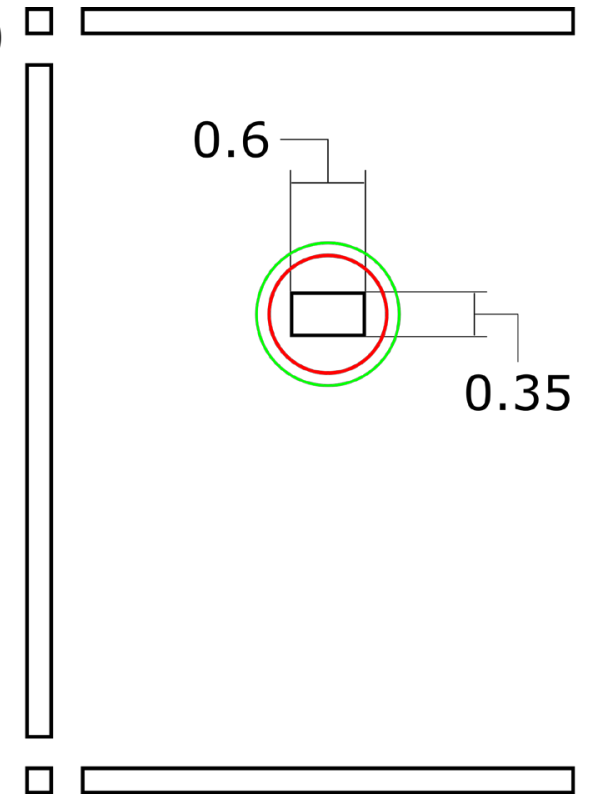

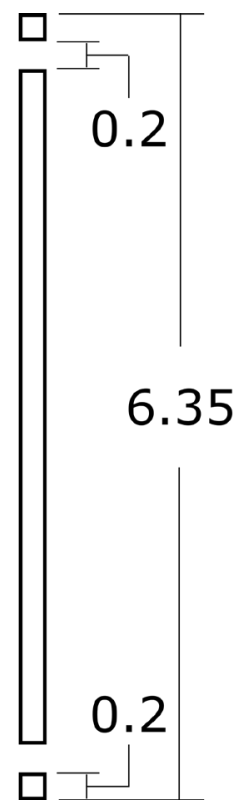

b)

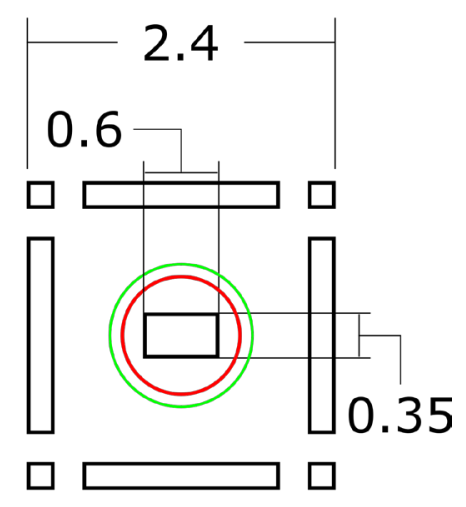

Figure 4.3: Figure 4.3: a) Mask design for large upper devices which fit the Protochips Liquid Stage holder. b) Mask design for the smaller lower devices which fit the Protochips Liquid Stage Holder.

device, creating the hermetically sealed chamber between these two o-rings. As a result, the Protochips Liquid Stage holder requires two different sized devices shown in Figure 4.3, and while the sealing strategy is slightly different the size of imaging area is similarly constrained by the size of the smallest o-ring, which has dimensions similar to the Hummingbird and DENS Solutions holders with an inner diameter of $1.2 \mathrm{~mm}$ depicted by the green circle in Figure 4.3a. The aperture for transmission is $1 \mathrm{~mm}$, which constrains imaging area and is depicted by the red circle in Figure 4.3a. Each device is $300 \mu \mathrm{m}$ in thickness for a total thickness of $600 \mu \mathrm{m}$, where this increase in thickness over the Hummingbird devices requires a slightly larger etching area for window formation at $0.6 \mathrm{~mm} x$ $0.35 \mathrm{~mm}$ for formation of a $300 \mu \mathrm{m} \times 50 \mu \mathrm{m}$ free standing membrane. The mask design for etching both upper and lower devices is shown in Figure 4.3a, to 

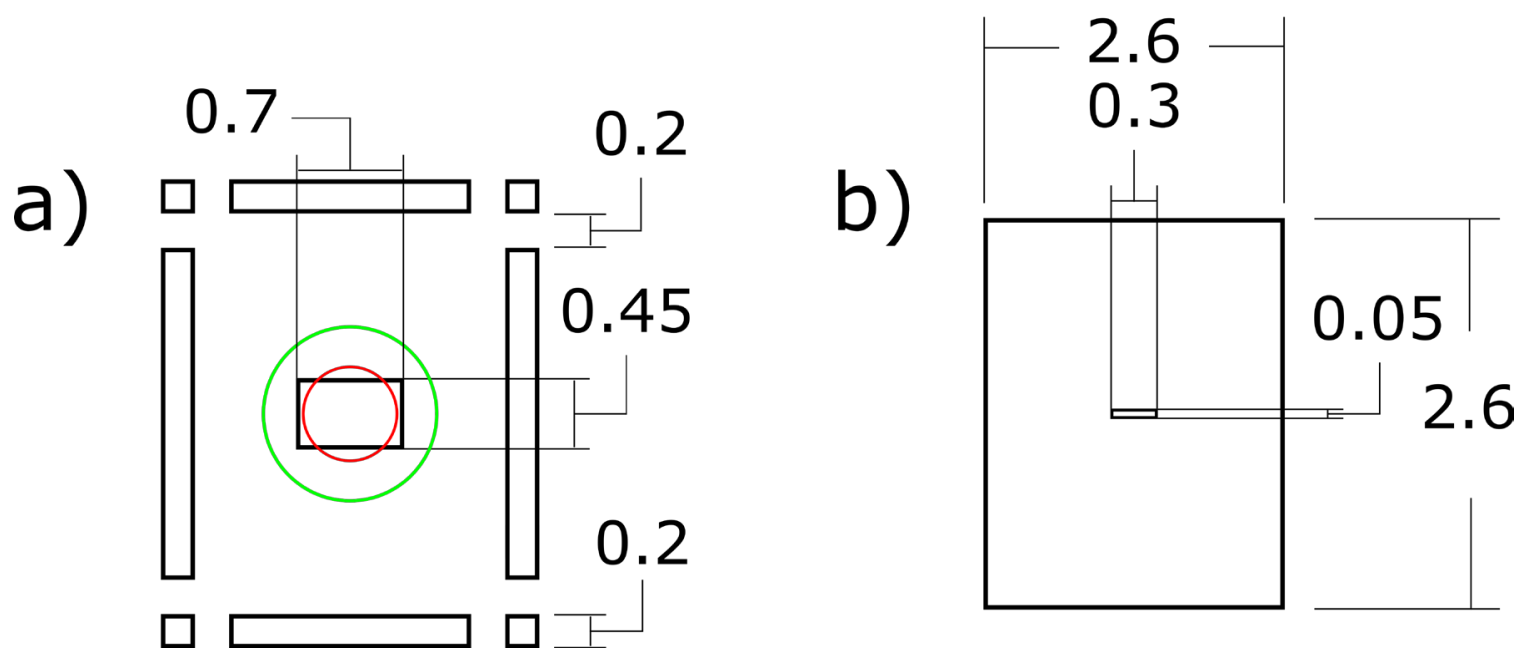

Figure 4.4: a) Mask design for large upper devices which fit the DENS Solutions Liquid Stage holder. b) Illustration of devices produced using the mask depicted in (a).

produce devices with dimensions detailed in Figure 4.3b, the upper device having dimensions of $5.95 \mathrm{~mm} \times 4.45 \mathrm{~mm}$ and the lower device having dimensions of $2 \mathrm{~mm} \times 2 \mathrm{~mm}$.

\subsubsection{Devices for the Dens Solutions LC-TEM Holder}

The DENS Solutions holder uses the same three o-ring sealing strategy as the Hummingbird holder, depicted in Figure 4.1, but constrained similarly by the oring sealing constraints and size of the holder aperture. The top and bottom devices are equal in size and are $2.0 \mathrm{~mm} \times 2.6 \mathrm{~mm}$, with a thickness of $400 \mu \mathrm{m}$ for each device for a total thickness of $800 \mu \mathrm{m}$. The small sealing o-ring has an inner diameter of $1.19 \mathrm{~mm}$ in diameter, depicted by the green circle in Figure 4.4a and is similar in size to the o-rings used by the Hummingbird and Protochips holders. The holder aperture however is considerably smaller when compared with the dimensions of the other holders at $0.65 \mathrm{~mm}$ diameter depicted by the red 
circle in Figure 4.4a, although this is enough area to fit a single window $300 \mu \mathrm{m}$ in length and $50 \mu \mathrm{m}$ in width. The increased thickness of the DENS Solutions devices necessitates the largest starting etch area of the three different devices at $0.45 \mathrm{~mm} \times 0.7 \mathrm{~mm}$. Figure 4.4 a shows the relevant mask dimensions for creating devices with a conventional fabrication protocol and Figure $4.4 \mathrm{~b}$ depicts the dimensions of the final devices.

\subsection{Fabrication of Single Window Devices}

The fabrication process for generation of a single window device for the above devices differs only in the design of the photomask for patterning and the duration of the $\mathrm{KOH}$ etch. Masks are created by copying the designs depicted in Figures $4.2 \mathrm{a}, 4.3 \mathrm{a}$, and $4.4 \mathrm{a}$ in a grid design to fill a four-inch $(100 \mathrm{~mm})$ diameter silicon wafer. An example of a completed mask to pattern for Hummingbird compatible devices is shown in Figure 4.5, where the spacing between each device allows for segmentation of the devices during etching of the window. A small amount of material is left near each corner to prevent the devices from falling apart during the etch process.

Four-inch wafers are purchased commercially double side polished and double side coated with low stress LPCVD silicon nitride. Silicon nitride film thickness can be $10-50 \mathrm{~nm}$ depending on the user needs for the devices, and low stress films are necessary to prevent wrinkling of the membranes after etching. Wafers are cleaned and dried under nitrogen, followed by spin coating 
a)

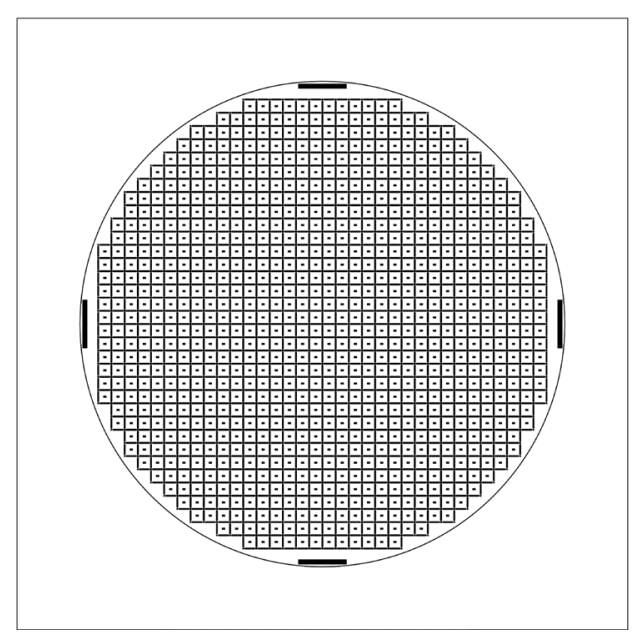

b)

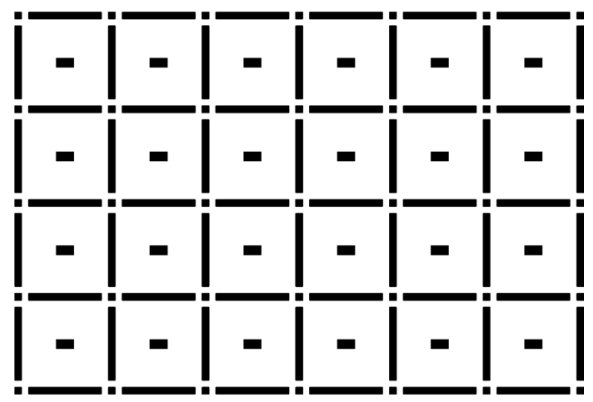

Figure 4.5: a) Illustration of a mask for replicating devices for the Hummingbird Liquid Stage holder (individual designs from Figure 4.2) across a 4-inch wafer. b) Subset of region from (a) showing the overlapping style of the borders of each device allowing for segmentation during etching.

hexamethyldisilane (HDMS) at 3000 rpm for 30 s to serve as an adhesion layer for the photoresist. The photoresist AZP 4620 is spin coated at $3000 \mathrm{rpm}$ for $60 \mathrm{~s}$ followed by soft baking the resist for two minutes at $70 \mathrm{C}$ and 3 minutes at $110 \mathrm{C}$. The wafer is then loaded into a mask aligner and aligned to the photomask containing the window and device border features illustrated in Figure 4.5. The wafer is then illuminated for 20 s followed by a rehydration incubation at room temperature for 30 minutes. Exposed photoresist is developed in AZ 400K developer in water at a 1:3 ratio for about 5 minutes or until features are completely developed. After development the wafer is hard baked at $180 \mathrm{C}$ for 30 minutes, where the wafer is not placed on a hotplate above $60 \mathrm{C}$ before ramping up to $180 \mathrm{C}$ and is not taken off until the hotplate has cooled to below $60 \mathrm{C}$ after baking to prevent cracking of the photoresist. The resist now covers all areas of the wafer which are not etched, the exposed silicon nitride is removed with an 
RIE etch and the remaining resist is stripped in 2xNanostrip at $110 \mathrm{C}$ for about 30 minutes or until all photoresist is removed from the wafer.

The wafer is then placed in a plastic holder and into a $40 \% \mathrm{KOH}$ bath at $80 \mathrm{C}$. Etching of the silicon is apparent from hydrogen bubble formation and windows are visible once etched by shining a light through the back of the etching wafer. Fully etched windows will allow for the transmittance of light when completed. The etch rate of silicon by $40 \% \mathrm{KOH}$ at $80 \mathrm{C}$ is about $1 \mu \mathrm{m} / \mathrm{min}(149)$ where the durations of etching for membrane formation for $200 \mu \mathrm{m}, 300 \mu \mathrm{m}$, and $400 \mu \mathrm{m}$ wafers will be approximately $200 \mathrm{~min}, 300 \mathrm{~min}$, and 400 min respectively. Simultaneously during window formation the borders of each device will be etched in order to separate the devices into their appropriate sizes. As such, care must be used when removing the wafer from the $\mathrm{KOH}$ bath in order to prevent the wafer from breaking apart due to excessive jostling of the wafer.

After removal, the etched wafer should be rinsed in water to remove excess $\mathrm{KOH}$ and then dried. If necessary, a 10 minute etch in $5 \mathrm{M} \mathrm{HCl}$ may be performed prior to rinsing with water to remove any metal contaminants picked up during the fabrication process, ensuring clean membranes for electron microscopy.

\subsection{Using Single Window Devices to Characterize Radiation Induced Chemistry Changes in Liquids}

Devices fabricated with the processing described in section 4.3 were tested for use in a Hummingbird Liquid Stage holder to determine the effectiveness and 
utility of the devices. Testing of devices entailed loading two devices within the tip of the holder with a small volume of liquid trapped between the devices, where imaging of the liquid was performed through the overlapping free standing silicon nitride membranes. While not shown, initial LC-TEM experiments identified membrane wrinkling and nitride surface contamination resulting from the fabrication process which were corrected through changes in the fabrication protocol. To demonstrate the science utility of the devices, experiments were devised to characterize the effect of the electron beam on liquid samples. As electrons are an ionizing radiation source and electron damage is known for materials and cryogenic samples, understanding how electrons interact with the liquid sample is key towards the ultimate project goal of imaging biological samples.

Initial experiments in the field have demonstrated the precipitation of nanoparticles from solution under electron beam irradiation (97). As existing projects within the group were interested in understanding the formation of nanoparticles for medical and energy applications, LC-TEM experiments on these samples were a convenient test case for characterizing electron-liquid interactions with the newly fabricated devices. The system of interest was the production of small nanoparticles of cerium oxide which have a number of applications including therapeutic properties due to their antioxidant characteristics in biological systems (209-213). Particles of less than $5 \mathrm{~nm}$ diameter appear to be preferably morphologies, and as such there is a need for a 
synthesis pathway to create a homogenous population. Electron irradiation of aqueous metal salt solutions has been demonstrated to reliably precipitate nanoparticles from solution that have a high degree of size uniformity. Precise control of the electron flux in combination with defined precursor chemistry can allow for some level of control over the size and morphology of the particles that are ultimately grown $(126,214)$.

The first step for a radiolysis driven growth experiment is to characterize electron beam conditions that can cause the reduction of the precursor solution to precipitate nanoparticles. The redox potential of the solution is dependent on the electron flux, where the illumination area is directly related to the corresponding electron flux. For STEM imaging, this also corresponds to the magnification and imaging field of view. Thus, the beam current must be appropriate at the optimum magnification that allows for visualization of the nanoparticles. Figure 4.6 shows an early experiment for finding conditions for imaging nanoparticles of ceria. Frames are taken during different time points during irradiation of a $\mathrm{CeNO}_{3}$ precursor solution at a $0.1 \mathrm{mM}$ concentration and an initial $\mathrm{pH}$ of 5.2. While some material can be seen to precipitate over the course of the experiment, discreet particles are not apparent at the magnifications used. Through further experiments, it was found that the precipitated nanoparticles only reached a maximum size of $\sim 2 \mathrm{~nm}$ diameter before aggregating and forming a thin film on the surface of the membrane (101). Intriguingly, these particles were found to be a phase of cerium oxide that normally only forms under basic $\mathrm{pH}$ conditions 


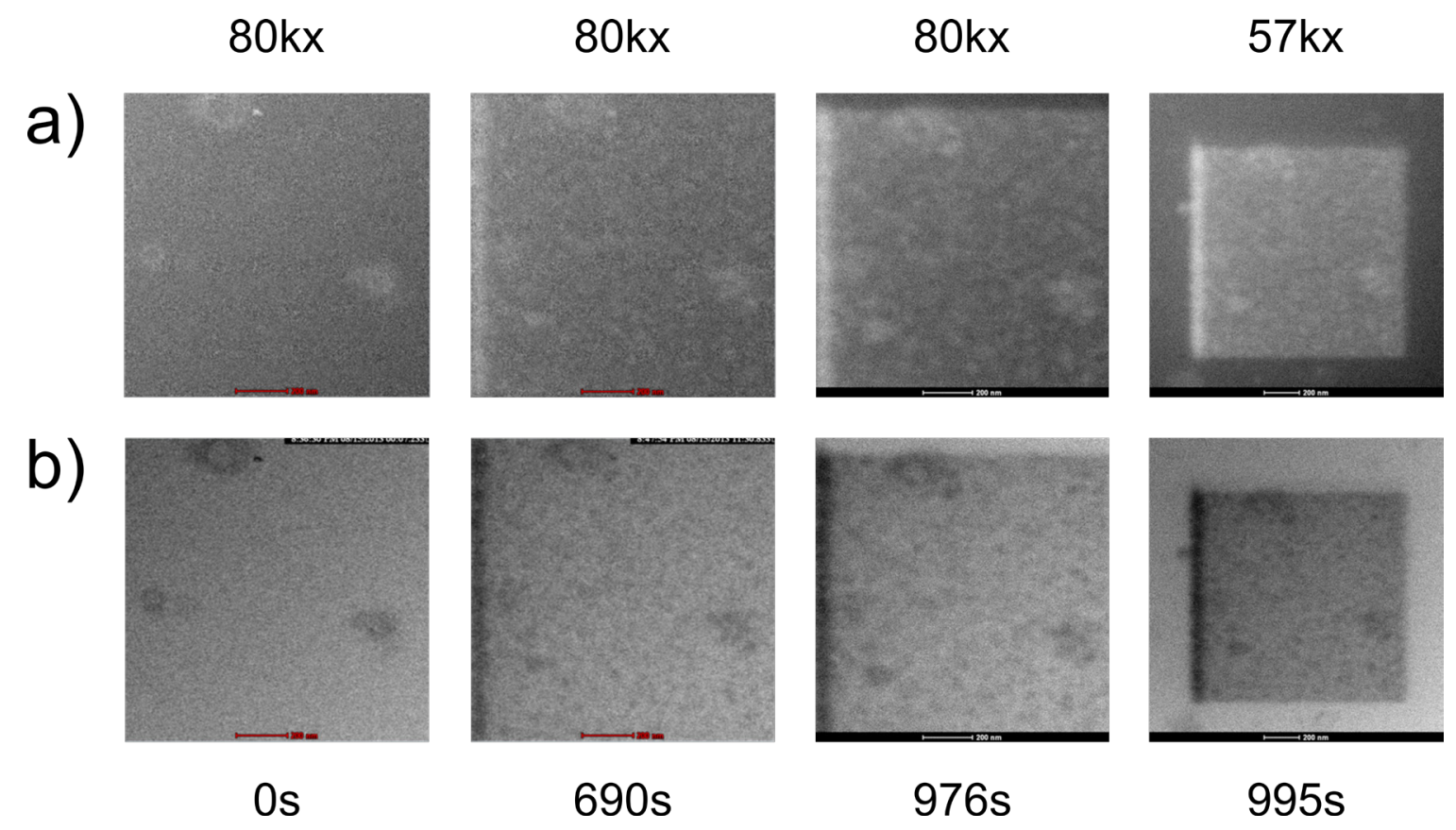

Figure 4.6: a) HAADF STEM image series of cerium nanoparticles precipitated under electron irradiation. b) BF STEM image series acquired simultaneously to the images in (a).

(101). Because previous work has suggested that for pure water the $\mathrm{pH}$ is always driven acidic regardless of the electron flux (215), we needed to further understand the chemistry of the system that would result in a phase that would not have been expected thermodynamically.

\subsubsection{Simulation of Radiolysis Driven Chemistry Changes}

The particle nucleation experiments above demonstrated an unexpected chemistry state of the system based on typical assumptions about electron beam driven solution changes. To understand these results, numerical simulation of radiolysis products can be used to explain how the solution chemistry resulted in a state that supported the formation of the observed cerium phase. Established 
simulation code was adapted to consider the cerium nitrate species of the precursor solution to simulate the effect of radiolysis on solution $\mathrm{pH}(215)$.

The nucleation and growth of metal nanoparticles from an aqueous metal salt precursor solution using LC-TEM has been well documented $(97,105,151)$, where the chemistry driving these redox reactions is predicted to be driven by electron beam radiolysis of the solvent water (215). The primary radiolysis products of pure water created upon irradiation by the electron beam are hydrated electrons ( $\left(\mathrm{eaq}^{-}\right), \mathrm{H}^{\circ}, \mathrm{OH}^{\circ}, \mathrm{HO}_{2}{ }^{\circ}, \mathrm{H}_{2}, \mathrm{H}_{2} \mathrm{O}_{2}$, and $\mathrm{H}_{3} \mathrm{O}^{+}$(215). These species react with each other and the surrounding water to form the steady state products shown in Chapter 2. The rate of production of these species under the electron beam has been determined known as the g-value, defined as the number of species formed per $100 \mathrm{eV}$ of energy absorbed (216), and are assumed to be homogenously distributed after $1 \mu$ s of irradiation (217). Along with the empirically determined g-values, known reaction constants for reactions between the radical species can be used in numerical simulations to determine the steady state products of radiolysis for a given incident dose (215). For pure water and doses commonly encountered for LC-TEM experiments it has been demonstrated that $\mathrm{pH}$ decreases with electron irradiation where the magnitude of change is dictated by the incident electron dose (215). By combining the known generation rate of radicals with the reaction constants from the relevant half reactions the steady state equilibria of radicals can be estimated through numerical simulation (215). For systems and precursors which have added metal 
salts, the additional half reactions can be added to the simulation to predict solution chemistry in more complex systems (126). This has been demonstrated for the precipitation of Au nanoparticles from a gold chloride precursor (126), suggesting it was possible to use the same system to describe the thermodynamics such that the phase of ceria observed could be formed.

Figure 4.7 shows simulations of the $\mathrm{pH}$ (concentration of $\mathrm{H}^{+}$ions) during electron irradiation. Figure 4.7a shows the $\mathrm{pH}$ change with different electron doses. Importantly, the dose for these calculations is expressed in Grays which is a measure of the absorbed dose. Only the inelastically scattered electrons contribute to radiolysis formation, the proportion of which compared to elastically scattered electrons is dependent on the thickness and atomic number of the sample. As such, the dose expressed in Grays is only linked to the electron flux through the average cross section of the sample. For all the relevant dose values for transmission electron microscopy the $\mathrm{pH}$ was calculated, and each reaches an equilibrium state by approximately $1 \mu$ s of irradiation time. Because the solute (in this case water) can be considered effectively infinite the conditions reached during electron irradiation revert back to the initial properties of the solution after the beam is turned off. Figure $4.7 \mathrm{~b}$ illustrates this behavior where the first $3 \mu \mathrm{s}$ shows the $\mathrm{pH}$ of the solution during electron irradiation. The beam is turned off (in simulation the generation of additional radical species of water is no longer considered) after $3 \mu$ s (indicated by the dashed red line) where the $\mathrm{pH}$ then rises back to its initial state over the course of several $\mu$ s. For both these cases of pure 
a)

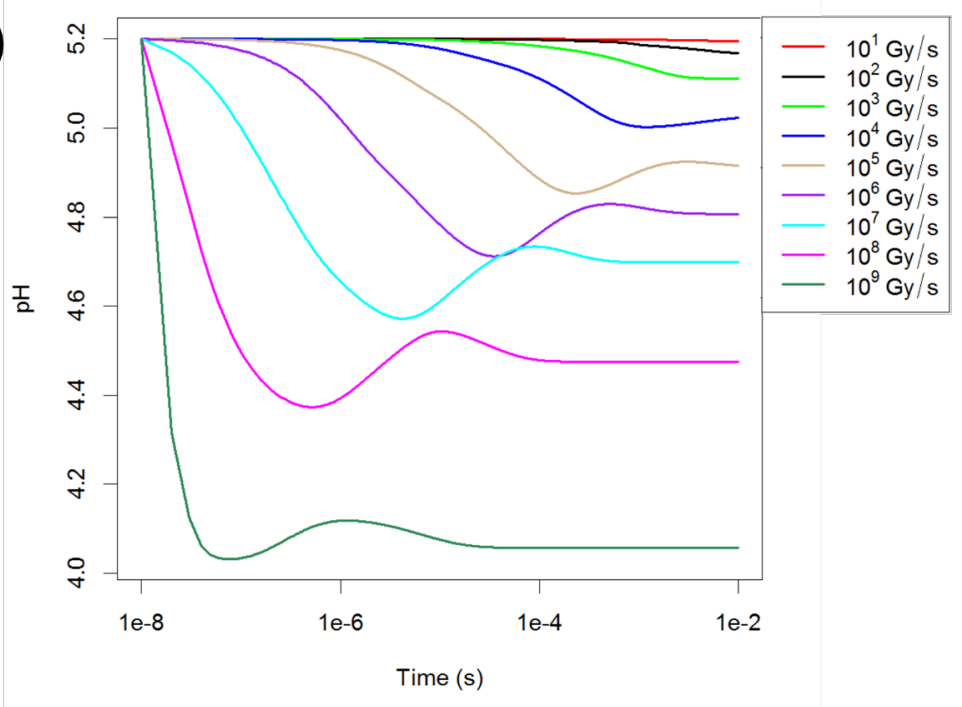

Beam On Beam Off

b)

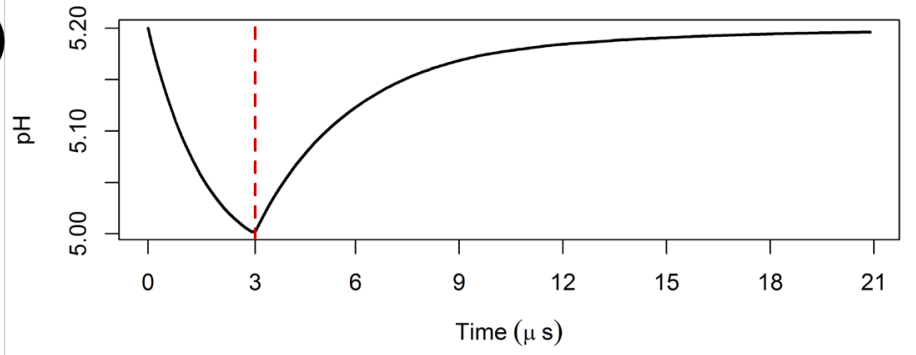

Figure 4.7: a) Simulation of the evolution of solution $\mathrm{pH}$ under different incident dose conditions. b) Simulation of $\mathrm{pH}$ under electron irradiation and then the relaxation period where the $\mathrm{pH}$ equilibrates after the beam is turned off. The dotted red line illustrates when the beam is turned off.

DI water, the $\mathrm{pH}$ decreases for all conditions. As mentioned before, the phase of cerium hydroxide that is reached is only thermodynamically stable at basic pH's, indicating that the additional $\mathrm{CeNO}_{3}$ in the precursor solution much change the chemistry enough to result in a basic $\mathrm{pH}$ during electron irradiation.

Table 1 shows the reactions and corresponding rate constants for atomic cerium with radicals that are produced through radiolysis. These rate constants were 
Cerium Reactions

(3)e-aq $+\mathrm{Ce} 3+\rightarrow \mathrm{Ce}$

$\mathrm{e}-\mathrm{aq}+\mathrm{Ce} 4+\rightarrow \mathrm{Ce} 3+$

$\mathrm{OH} \cdot+\mathrm{Ce} 3+\rightarrow \mathrm{Ce} 4++\mathrm{OH}-$

$\mathrm{H} \cdot+\mathrm{Ce} 4+\rightarrow \mathrm{H}++\mathrm{Ce} 3+$

$\mathrm{O} \cdot-+\mathrm{Ce} 3+\rightarrow \mathrm{Ce} 4++\mathrm{OH}-$

$\mathrm{HO} 2 \cdot+\mathrm{Ce} 3++\mathrm{H}+\rightarrow \mathrm{H} 2 \mathrm{O} 2+\mathrm{Ce} 4+$

$\mathrm{HO} 2 \cdot+\mathrm{Ce} 4+\rightarrow \mathrm{Ce} 3++\mathrm{H}++\mathrm{O} 2$

$\mathrm{Ce}+(3) \mathrm{H} 2 \mathrm{O} \rightarrow \mathrm{Ce} 3++(3) \mathrm{OH}-+(3 / 2) \mathrm{H} 2$

\section{Rate Constant $\left(25^{\circ} \mathrm{C}\right)\left(\mathrm{M}^{-1} \cdot \mathrm{s}^{-1}\right)$}

$k=1.0 \times 10^{\wedge} 9$

$k=6.6 \times 10^{\wedge} 10$

$k=3.0 \times 10^{\wedge} 8$

$k=6.5 \times 10^{\wedge} 7$

$k=7.2 \times 10^{\wedge} 8$

$k=7.3 \times 10^{\wedge} 5$

$k=2.7 \times 10^{\wedge} 6$

$k=1.0 \times 10^{\wedge} 9$

incorporated into the numerical simulation code, and simulations were run to check the evolution of $\mathrm{pH}$ with the included Ce reactions. Figure $4.8 \mathrm{a}$ shows the $\mathrm{pH}$ evolution of $\mathrm{DI} \mathrm{H}_{2} \mathrm{O}$ while Figure $4.8 \mathrm{~b}$ shows the $\mathrm{pH}$ evolution for an identical electron dose but including the Ce reactions in table 1 . When the Ce products are included, the $\mathrm{pH}$ rises for the given dose, $\sim 10^{4} \mathrm{~Gy} / \mathrm{s}$ (the dose was equivalent to the electron flux used for imaging experiments). Increasing the electron flux (and corresponding absorbed dose), results in the $\mathrm{pH}$ initially decreasing and still rising after several nano seconds.

If the dose is high enough, the $\mathrm{pH}$ equilibrium is ultimately more acidic than the initial starting $\mathrm{pH}$. This relationship is shown in Figure 4.8c. The rise in $\mathrm{pH}$ observed when incorporating the Ce reactions in table 1 is likely due to the excess of $\mathrm{OH}^{-}$which is produced through the cycling of Ce oxidation state which 

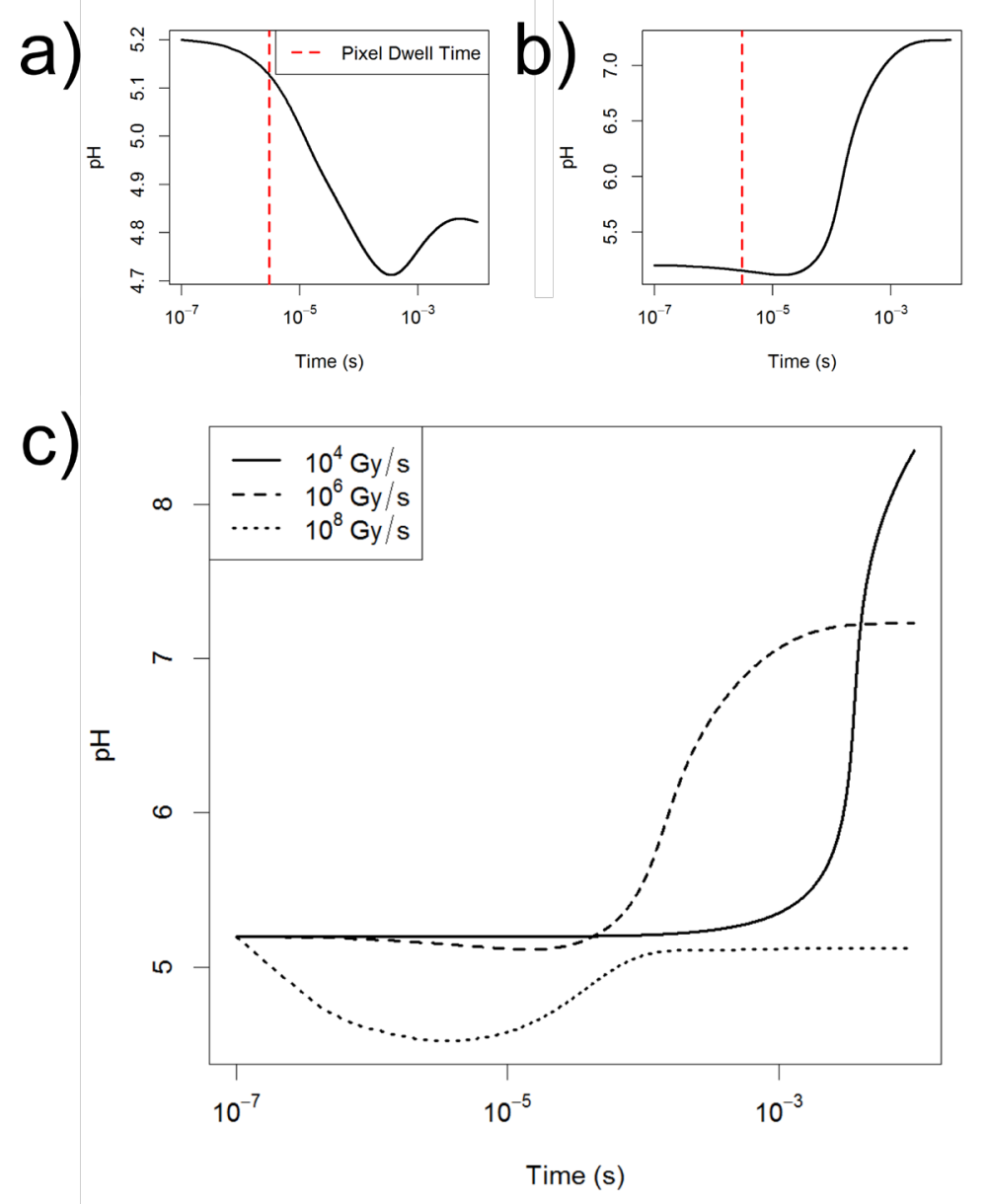

Figure 4.8: a) Simulation of $\mathrm{pH}$ for pure water under electron irradiation. b) Simulation of solution $\mathrm{pH}$ for an aqueous solution of cerium nitrate. c) Simulation of solution $\mathrm{pH}$ for an aqueous solution of cerium nitrate under varying dose conditions.

results in a consumption of produced $\mathrm{H}+$ and generates $\mathrm{OH}$. While the $\mathrm{pH}$ is seen to rise, for the starting $\mathrm{pH}$ of 5.2 the equilibrium $\mathrm{pH}$ is only around 7 , still not high enough to thermodynamically favor the formation of the phase of cerium hydroxide observed in the LC-TEM experiments. It should be noted that only the Ce species were incorporated in the numerical simulations for calculating $\mathrm{pH}$. $\mathrm{NO}_{3}$ was also present in the precursor solution, although the inclusion of reaction 
constants for every possible $\mathrm{NO}_{3}$ reaction would have considerably increased the complexity of the simulations. Furthermore, not all reaction rates are known or publicly available for every possible reaction, further complicating their inclusion.

$\mathrm{NO}_{3}{ }^{-}+10 \mathrm{H}^{+}+8 e_{a q}{ }^{-} \rightarrow \mathrm{NH}_{4}{ }^{+}+3 \mathrm{H}_{2} \mathrm{O}$

$\mathrm{NO}_{3}^{-}+7 \mathrm{H}_{2} \mathrm{O}+8 e^{-}{ }_{a q} \rightarrow \mathrm{NH}_{4}^{+}+10 \mathrm{OH}^{-}$

Reaction 1 and reaction 2 are both reactions of $\mathrm{NO}_{3}$ with aqueous electrons would produce an excess of $\mathrm{OH}$ - which would drastically drive the $\mathrm{pH}$ basic. It may very well be that the inclusion of the $\mathrm{NO}_{3}$ species is enough to substantially increase the $\mathrm{pH}$ to thermodynamically favor the phase of cerium hydroxide that was observed.

The use of numerical simulation to characterize the chemistry of the liquid sample during electron irradiation is an important step in characterizing electron beam-water interactions. While biological samples will ultimately be far more complex than the systems described here, they provide a good foundation for future studies trying to understand the role of the electron beam in driving dynamics with LC-TEM. 


\section{Chapter 5: Fabrication of Multiwindow Nanofluidic Devices and the Role of Electron Irradiation History in Interpretation of LC-TEM Results}

\subsection{Introduction}

In chapter 4 we demonstrated the utility of LC-TEM for imaging the dynamics of nanoparticle growth at high spatial resolution in conjunction with numerical simulations to explain the chemistry behind the products precipitated from the electron beam (101). However, the replication of experiments which utilize the single window devices described there is difficult due to intrinsic limitations surrounding the design and geometry of the devices themselves (103). These limitations constrain the type and scope of applications which may be investigated with the technique and as such, overcoming them is critical to expanding science applications. In order to accelerate the impact of the technique we identified primary design areas which were the source of experimental bottlenecks. In this chapter I describe the design, fabrication, and optimization of new devices which overcome the limitations of currently available commercial solutions.

\subsection{Limitations of Single Window Devices}

\subsubsection{Bulging of Membranes}

One of the consequences of imaging hermetic chambers in a TEM is the pressure differential that occurs between the high vacuum state of the instrument (typically near $10^{-6} \mathrm{mbar}$ ) and the significantly higher pressure of our ambient 
environmental samples (generally $1 \mathrm{~atm}$ ). As the described membranes used for imaging are quite thin relative to their width and length (generally 10 s of nanometers in thickness and microns in area), their ability to resist the outward pressure of the liquid sample against the vacuum of the column is limited. In practice this results in significant outward bulging of the membranes, and can be as much as 100 s of nanometers towards the center of the films (124). This bulging results in a gradient of thickness increase across the imaging window, with the maximal thickness in the center. Figure 5.1a shows a BF-TEM image of a typical liquid cell depicting the effect of this window bulging on image contrast, where the corners are the thinnest sections of the imaging area and allow for the highest transmittance of electrons. Towards the center of the window as thickness increases contrast is reduced due to increased inelastic collisions which scatter electrons at high angles. The magnitude of this bulging can be quantified using EELS, where the thickness at probe locations can be determined by collecting spectra and calculating the log ratio between the integrated signal of the zero loss peak and the plasmon. Figure $5.1 \mathrm{~b}$ shows a plot of measured thicknesses using EELS across a single window inside of the microscope displaying characteristic bulging.

The practical effect of this bulging induced thickness gradient is that most experiments are limited to the corners of the windows (218). Primarily this is to maximize contrast and resolution, and signal to noise, contrast, and resolution are all reduced with increasing thickness (219). Additionally, as discussed in 

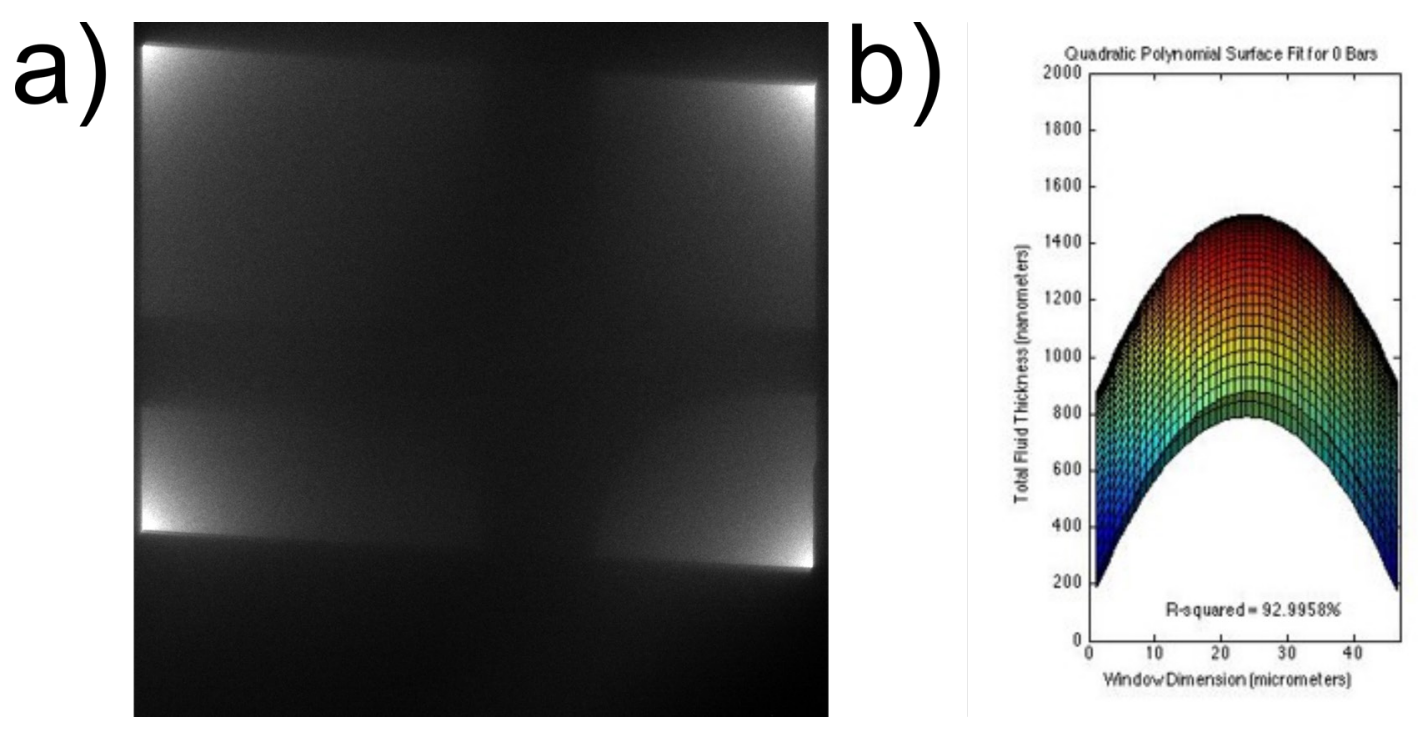

Figure 5.1: a) Low magnification HAADF STEM image of a LC-TEM window depicting a contrast gradient towards the center of the window indicating increasing thickness. b) Illustration of membrane bulging based on EELS thickness measurements taken from a window region similar to the one shown in (a).

chapter 4 , for experiments which require precise control over the chemistry of the solution or precursor a gradient of thicknesses could cause changes in concentration of beam generated radicals which can significantly impacting observed results. Thus, in order to ensure that the thicknesses are effectively equivalent for comparison of replicate experiments or observations, membrane bulging effectively limits observations to the corners of the windows.

\subsubsection{Limited Imaging Area}

As illustrated in chapter 4 the free-standing silicon nitride membrane formed from the standard fabrication protocol results in a single window which is $50 \mu \mathrm{m} \times 300$ $\mu \mathrm{m}$, shown in Figure 5.2a. During assembly, the membranes between two devices can be aligned such that they are oriented in the same direction as 
a)

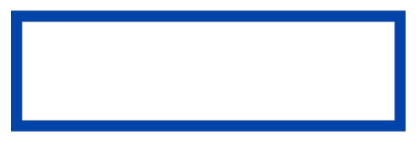

b)

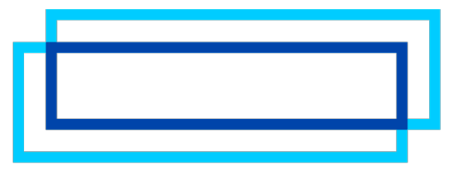

c)

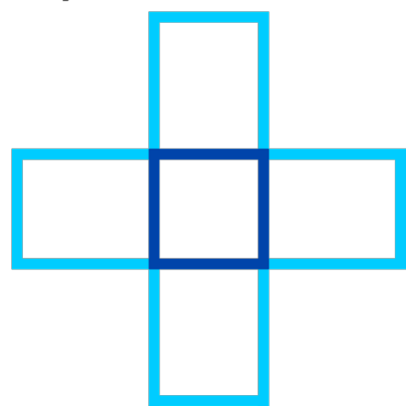

Figure 5.2: a) Illustration of a single rectangular window region for LC-TEM. b) Illustration of a parallel window alignment strategy where slight misalignment between windows results in non-equivalent thicknesses at each window corner. c) Illustration of perpendicular alignment strategy which creates 4 window corners of effectively equivalent thickness.

shown in Figure 5.2b. However, as illustrated in Figure 5.2b, where the light blue window is one window and the dark blue window is the other, the imprecision of alignment between the two windows can be as much as 10-20 $\mu \mathrm{m}$ which results in overlapping of the imaging area. While this orientation still results in the largest potential viewing area, the membrane bulging described in section 5.2.1 results in different effective thicknesses at each window corner. This window orientation then makes quantitative comparisons between successive imaging experiments performed in different locations complicated or impossible. Figure 5.2c shows the alternative assembly where one window is rotated $90^{\circ}$ with respect to the other resulting in the crossed arrangement shown in Figure 5.2c. This arrangement is resistant to variable alignment, where the thicknesses at each corner will be effectively equivalent when taking into account window bulging. While this arrangement is optimal for quantitative comparison of experiments, the end result is that there is a significant reduction in available imaging area for experiments. Typically, with a single window device, a maximum of four experiments may be 
performed on a single device which drastically reduces the throughput of the technique, and as described in the next section, results between separately assembled samples proves highly difficult in practice.

\subsubsection{Thickness Variation}

As a result of reduced imaging area, for replication of datasets or collection of multiple images of a sample for characterization several different samples must be loaded and assembled into a set of devices. In theory the thickness of the liquid layer between the two silicon nitride membranes can be dictated by the use of spacer material patterned on the surface of one, or both, of the devices (77). However, this thickness can be increased by environmental contaminants, a nonaqueous sample sitting on top of the spacer material, or a non-aqueous sample may clump together forming a particle which dictates the nominal thickness of the assembled liquid cell. Despite careful control of loading and the quality of the sample highly variable thicknesses are typical for otherwise identical samples.

Figure 5.3 illustrates this variability and the effect it has on systems studying the chemistry of nucleation and imaging of biological samples. Figure 5.3a shows frames from experiments precipitating silver nanoparticles from an aqueous precursor of $0.1 \mathrm{mM}$ silver nitrate from three different assembled liquid cells. Frames are shown at three minutes into irradiation and after 10 minutes of irradiation. For each assembled sets of devices and experiments the spacer thickness was the same and the imaging conditions used, electron flux, field of 
$3 \min$
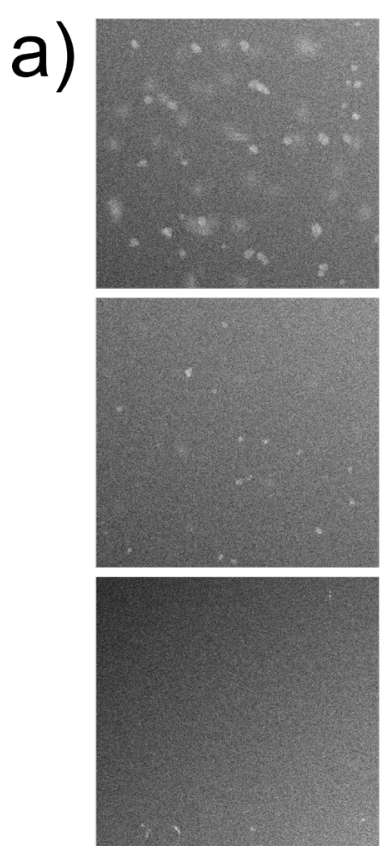

$10 \min$
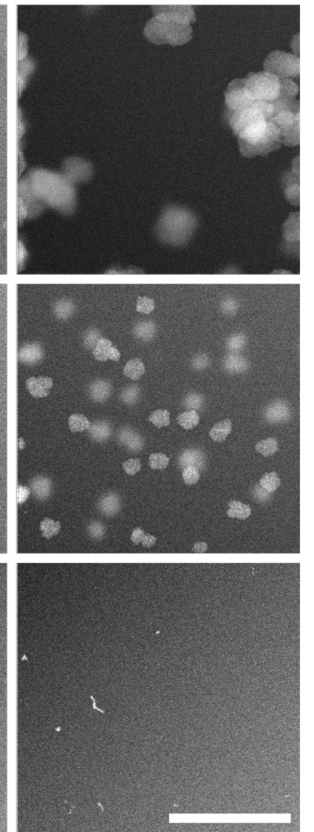

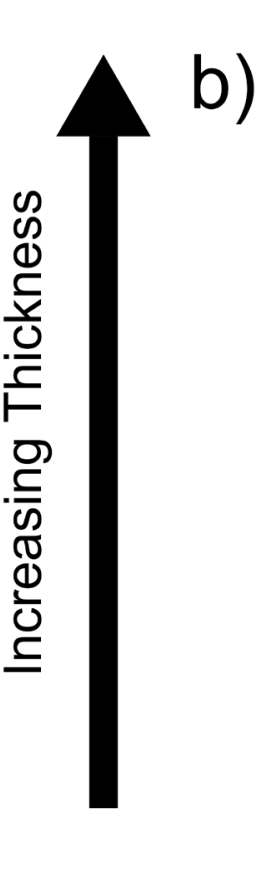

High Mag
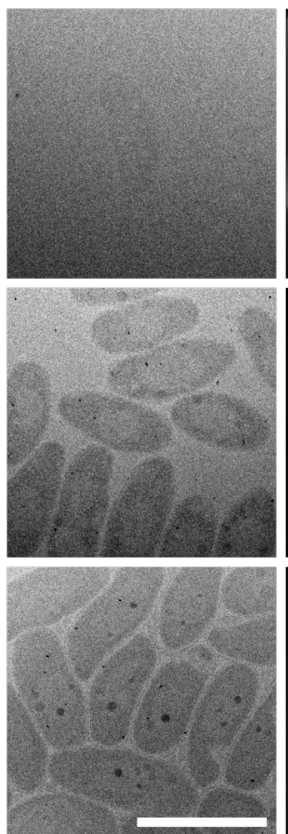

Low Mag
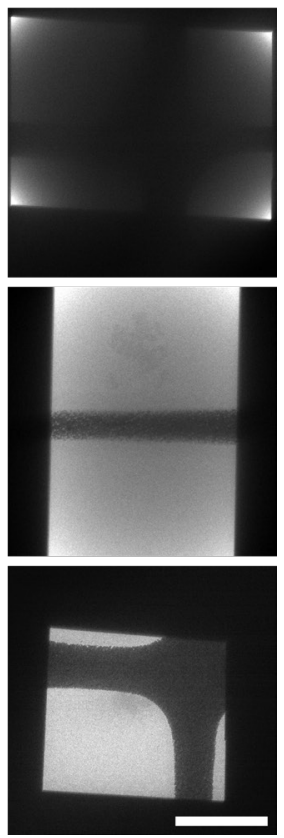

Figure 5.3: a) Frames from three different nanoparticle growth experiments where the precursor and imaging conditions were identical between each experiment and the only difference was thickness. Different growth kinetics are apparent between the different samples as a result of thickness changes. b) Bacteria imaged with LC-TEM at low and high magnifications depicting the effect of increasing thickness on resolution, contrast, and signal to noise ratio. Scale bar in (a) is $50 \mathrm{~nm}$ and in (b) is 1 and $10 \mu \mathrm{m}$.

view, and time, were all the same. The only variable not controlled between these experiments was the thickness, where significant changes in nucleation behavior was observed for different liquid thickness conditions. Figure 5.3b shows the effect of thickness on variability on imaging whole cells, where an increase in thickness between otherwise identical samples results in a significant loss of signal to noise, contrast, and resolution for increasing thicknesses. Unfortunately, despite the importance sample thickness plays in the outcome of LC-TEM experiments it remains a highly variable parameter that is difficult to control especially for non-aqueous samples which have a tendency to clump together. This variability amplifies the factor of luck for obtaining multiple samples 
with similar liquid thicknesses, and substantially reduces the throughput of the technique.

\subsubsection{Bypass of Flow}

For commercially available holders which have the capability to flow fresh liquid to the imaging area the option of flowing a non-aqueous sample to the imaging area may be attractive for reducing the chances of sample clumping or nonuniform distribution over the device surface. Furthermore, while many nanoparticle growth experiments are initiated using the electron beam as a reducing agent the ability to flow two mixing samples which result in particle growth (125) or environmental stimuli to a biological sample (112) is a potential way to visualize dynamics near initialization of the reaction. For all of these cases however there is no direct path of the sample to the imaging area, as depicted by Figure 5.4. Figure 5.4a shows a cross section of a generic LC-TEM holder using 3 o-rings to achieve hermeticity, while Figure $5.4 \mathrm{~b}$ shows a top down view of the same assembly. The flow inlet for liquid enters the hermetic chamber, where its outer constraints are the large, out o-ring, and inner constraints are the small inner o-rings surrounding the windows. As such, there is nothing forcing the liquid sample to flow through, rather than around, over, or below, the devices.

Furthermore, even if sample does flow between the devices there is no incentive for the sample to flow over the imaging area which is small relative to the rest of the surface area of the device. The result of this is that there can be significant flow of sample around rather than through the imaging windows. Especially for 
a)

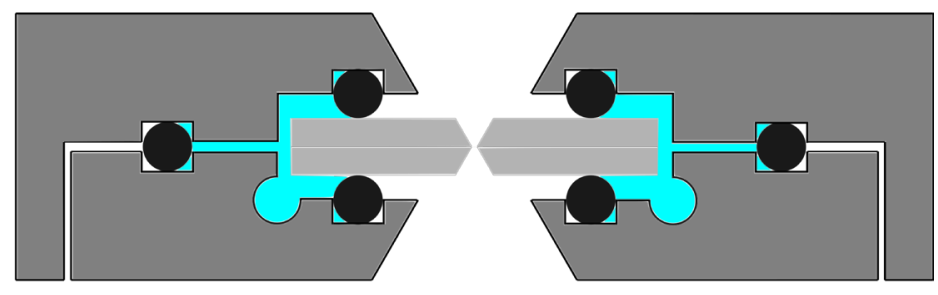

b)

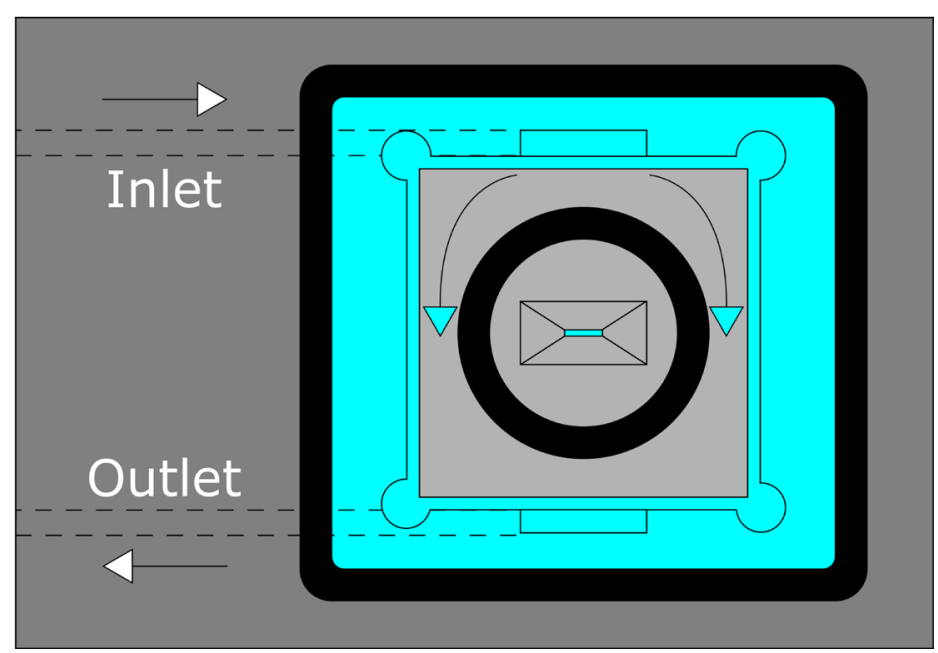

Figure 5.4: a) Cross sectional illustration of a flow holder depicting the bypass flow channels where liquid can flow around the sample. b) Top down illustration of the same holder in (a) aiding visualization of how flow can pass around, or over, the sample.

samples which are at or near the thickness separating the two devices, often 1 $\mu \mathrm{m}$ or less, the path of least resistance is around the devices which have channels 10 s to 100 s of microns in dimensions. For experiments which utilize these flow capabilities to flow already nucleating particles (125), it is unclear how representative the samples which reach the imaging windows are of the bulk sample, or if significant "filtering" of the sample may occur due to the drastic difference in channel dimensions. This limits significantly the type and scope of experiments which may be performed with LC-TEM. 


\subsection{Design of Multiwindow Devices}

\subsubsection{Hummingbird Devices}

The mask dimensions for fabrication of five window devices compatible with the Hummingbird Liquid Stage is shown in Figure 5.5a. The starting etch area for the windows are rectangles $40 \mu \mathrm{m}$ in width and $700 \mu \mathrm{m}$ in length. Each window is spaced $110 \mu \mathrm{m}$ edge to edge and all five windows are centered within the device. Channels $40 \mu \mathrm{m}$ in width are also patterned defining the edges of the device and section the devices apart during window formation to form devices. Patterned borders result in devices which are approximately $2.52 \mathrm{~mm}$ on each side when fabricated. $100 \mu \mathrm{m}$ segments are left out at each of the corners in order to "perforate" the devices so they stay together during etching. Figure $5.5 \mathrm{~b}$ depicts the final devices where each is $2.52 \mathrm{~mm} \times 2.52 \mathrm{~mm}$ and contain five windows, each of which is approximately $40 \mu \mathrm{m}$ in width and $680 \mu \mathrm{m}$ in length. The green and red circles in Figure 5.5a depict the size of the inner diameter of the sealing o-ring (1.24 $\mathrm{mm}$ inner diameter) and the diameter of the aperture in the holder $(0.79 \mathrm{~mm}$ diameter) respectively with the holder aperture constraining the area through which electrons may be transmitted. The final windows formed are approximately $40 \mu \mathrm{m}$ in width and $680 \mu \mathrm{m}$ in length, which fits within the inner diameter of the sealing o-ring and within the aperture diameter. In theory this results in a $5 \times 5$ grid of available windows when assembled, although often due to imprecision of device alignment one or several of the windows may become occluded by the aperture of the holder. 

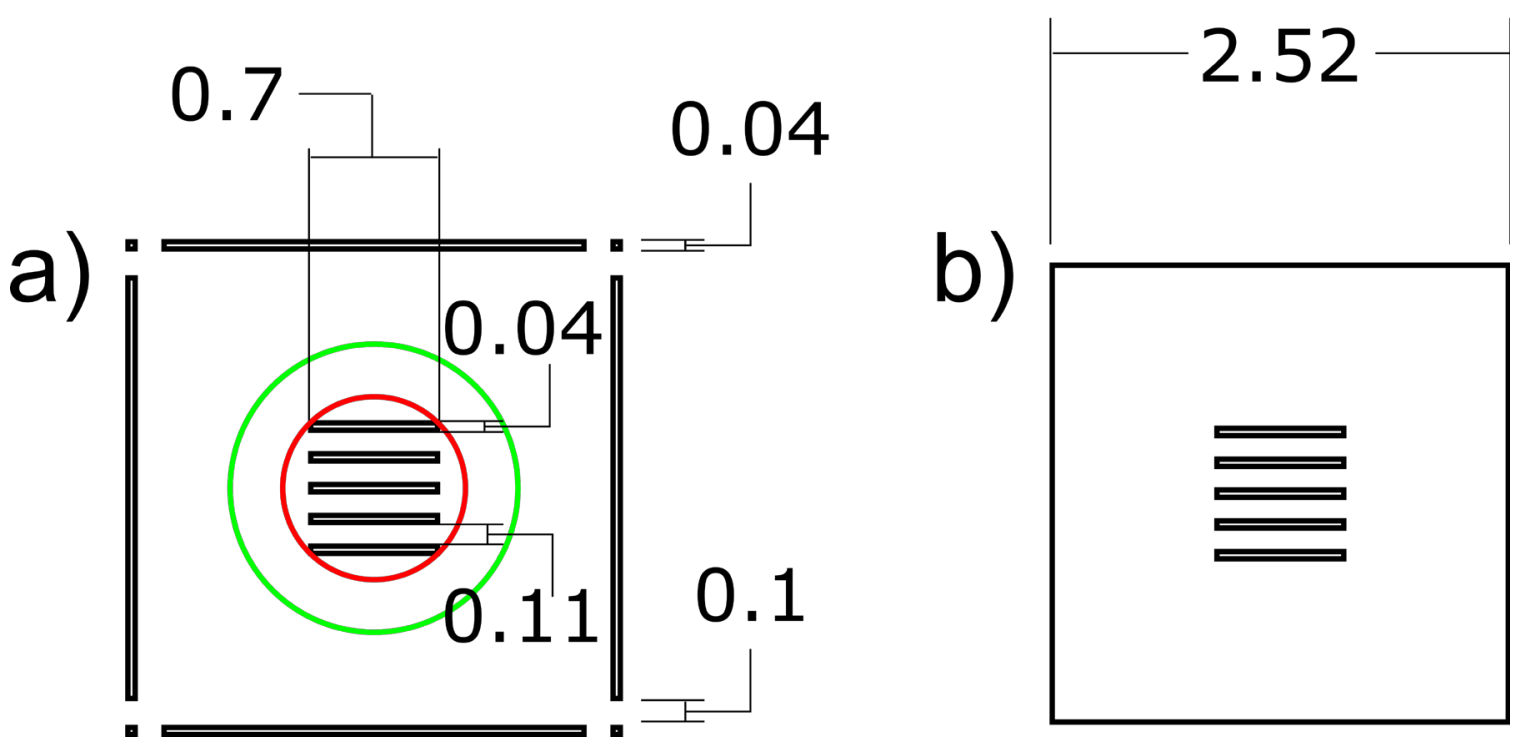

Figure 5.5: a) Mask design for multiwindow devices which fit the Hummingbird Liquid Stage holder. b) Illustration of devices produced using the mask depicted in (a).

\subsubsection{Protochips Devices}

Figure $5.6 \mathrm{a}$ and $5.6 \mathrm{~b}$ shows the mask design for fabrication of the upper and lower devices for use in the Protochips Liquid Stage holder. Similar to the Hummingbird devices the outlines of each device for the Protochips holder is a $40 \mu \mathrm{m}$ width channel with $100 \mu \mathrm{m}$ length perforations at the corners. The upper device for the Protochips device is larger, with a final size of $4.45 \mathrm{~mm}$ in width and $5.95 \mathrm{~mm}$ in length shown in Figure 5.6d. The window position is offset from the long axis and centered in the short axis, with the center window being 2.3 $\mathrm{mm}$ from the leading edge of the device as shown in Figure 5.6a. The etch area for each window is $40 \mu \mathrm{m}$ in width and $700 \mu \mathrm{m}$ in length, each separated $110 \mu \mathrm{m}$ edge to edge. These fit within the small sealing o-ring ( $1.2 \mathrm{~mm}$ inner diameter) and within the aperture of the holder $(1 \mathrm{~mm})$ depicted by the green and red 
a)

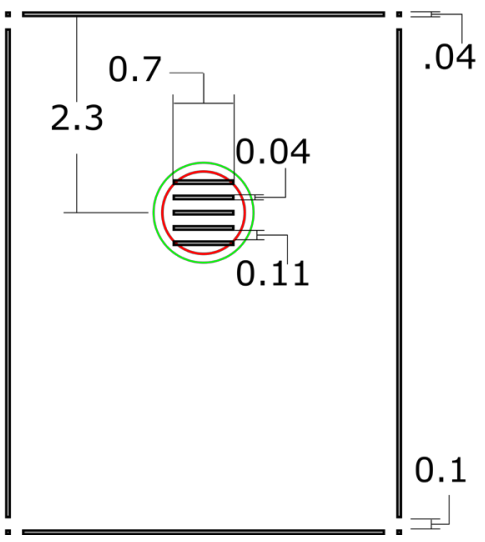

2.08

b)

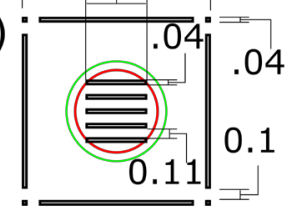

c)

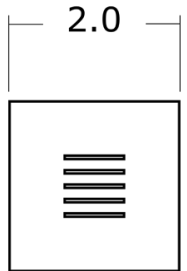

d)

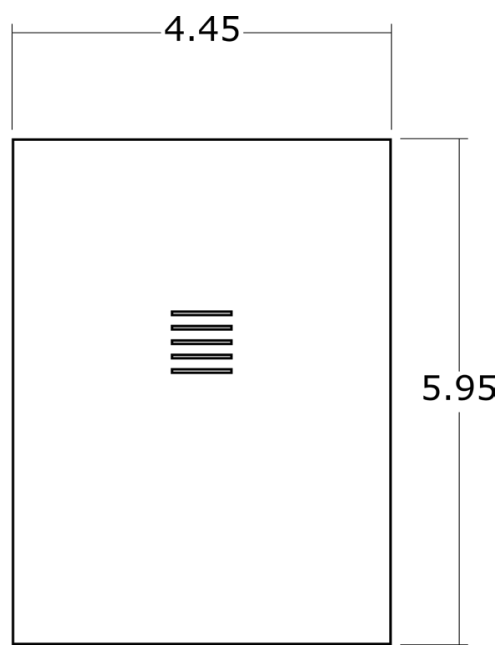

Figure 5.6: a) Mask design larger upper for multiwindow devices which fit the Protochips Liquid Stage holder. b) Mask design for the smaller lower multiwindow devices which fit the Protochips Liquid Stage holder. c) Illustration of devices produced using the mask depicted in (b). d) Illustration of devices produced using the mask depicted in (a).

circles respectively. The lower device for the Protochips holder is smaller, with final dimensions of $2.0 \mathrm{~mm}$ in width and $2.0 \mathrm{~mm}$ in height shown in Figure $5.6 \mathrm{c}$. The dimensions for the border and window area is identical to the larger upper device but is centered equally from each edge of the smaller device.

\subsubsection{Dens Solutions Devices}

Figure 5.7a shows the mask design for devices used in the Dens Solutions Liquid Stage holder. The border of each device is formed by etching a $40 \mu \mathrm{m}$ channel around the outline of the device as has been described for the other devices for the Hummingbird and Protochips holders. Each corner is perforated with a 100 $\mu \mathrm{m}$ spacer to prevent the devices from falling apart during etching. The green and red circles in Figure 5.7a show the size of the inner diameter of the small sealing o-ring and the diameter of the aperture of the holder which are $1.19 \mathrm{~mm}$ 


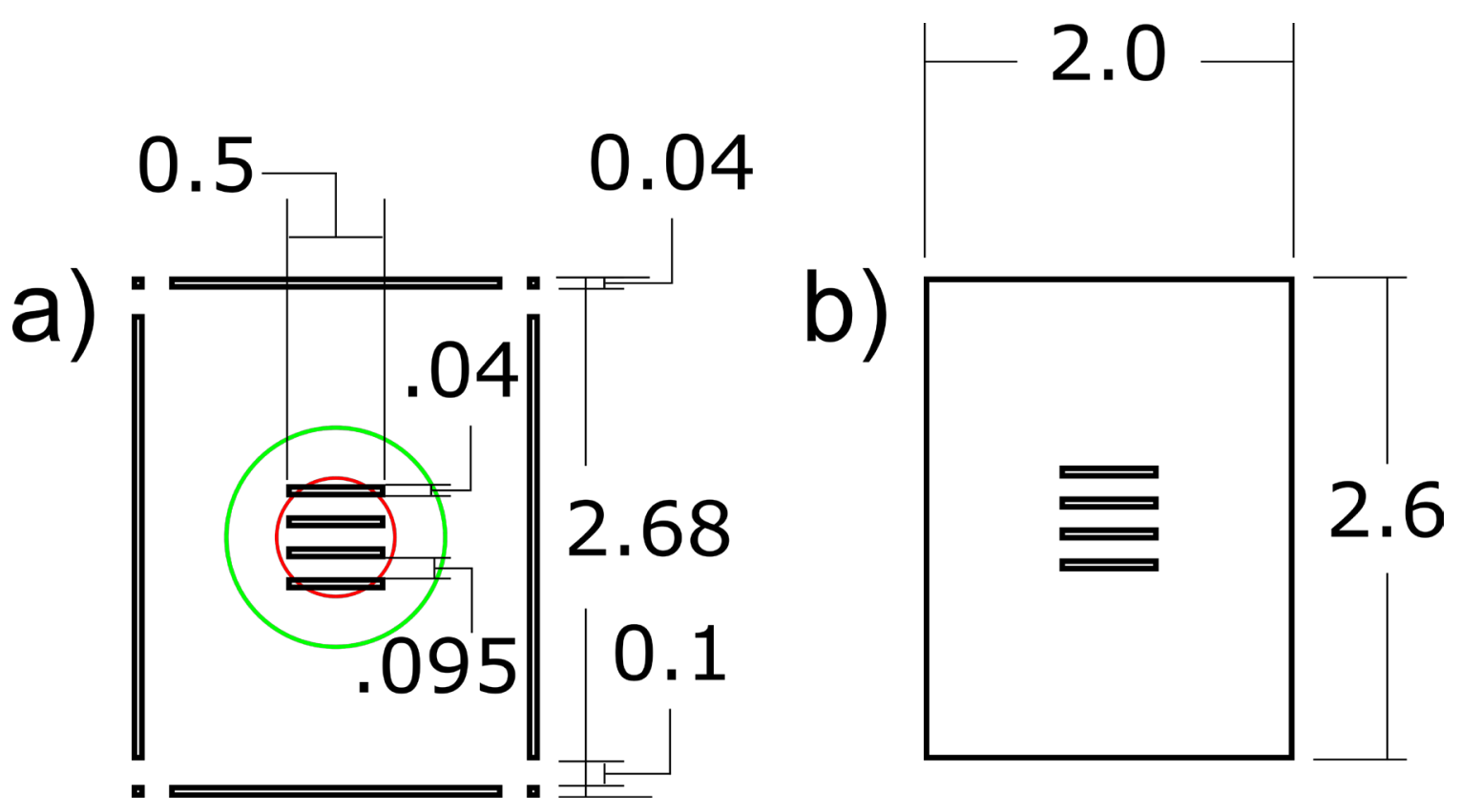

Figure 5.7: a) Mask design for multiwindow devices which fit the DENS Liquid Stage holder. b) Illustration of devices produced using the mask depicted in (a).

and $0.65 \mathrm{~mm}$ in diameter respectively. The aperture for the Dens Solutions holder is the smallest of the three commercially available holders, and it is difficult to fit five windows within this area as a result. Separation between windows is important to provide structural support of each individual window, and while five windows could be fit the spacing between each would be little enough that the silicon separating the windows could be easily damaged without especially careful handling of the devices, reducing their throughput. With four windows, $95 \mu \mathrm{m}$ edge to edge separation of each window is possible, compared to the $110 \mu \mathrm{m}$ edge to edge separation used in the Protochips and Hummingbird holder designs. The four windows are centered within the device borders and are equidistant from each edge. The etch area for each window is $500 \mu \mathrm{m}$ in width 
and $40 \mu \mathrm{m}$ in width, for final window dimensions of approximately $40 \mu \mathrm{m}$ in width and $480 \mu \mathrm{m}$ in length. The final device dimensions are $2 \mathrm{~mm}$ in width and $2.6 \mathrm{~mm}$ in height shown in Figure 5.7b.

\subsection{Fabrication of Multiwindow Devices}

The fabrication protocol described in Chapter 4 for fabrication of single window devices utilizing a $\mathrm{KOH}$ etch through the full thickness of the substrate results in anisotropic etching of the silicon along the [111] planes. For the [100] oriented Si wafers used for fabrication this results in inward tapered pits that are pyramidal in structure. To fit more windows within the same area etching must occur at a higher aspect ratio, where the ideal profile of the etching is vertical. Deep reactive ion etching (DRIE) is an etching process which uses a plasma to facilitate etching and is known as "dry etching" since it does not take place in a liquid environment as other wet etching techniques do such as $\mathrm{KOH}$ etching. The Bosch process allows for this high aspect ratio etching, where nearly vertical side walls can be achieved through serially alternating between deposition of a Teflon layer and then etching with an oxygen plasma. Figure 5.8 illustrates the difference in etching profile between the $\mathrm{KOH}$ based process described in Chapter 4 and the Bosch etch based process that is used to fabricate multiple windows described below. Figure 5.8a is a cross section of a device (not shown to scale) which has been fabricated with $\mathrm{KOH}$ illustrating the anisotropic etching along the [111] planes at a $54.7^{\circ}$ angle. This limits the number of etching regions which can be placed within the constraints of the inner diameter of the sealing o- 

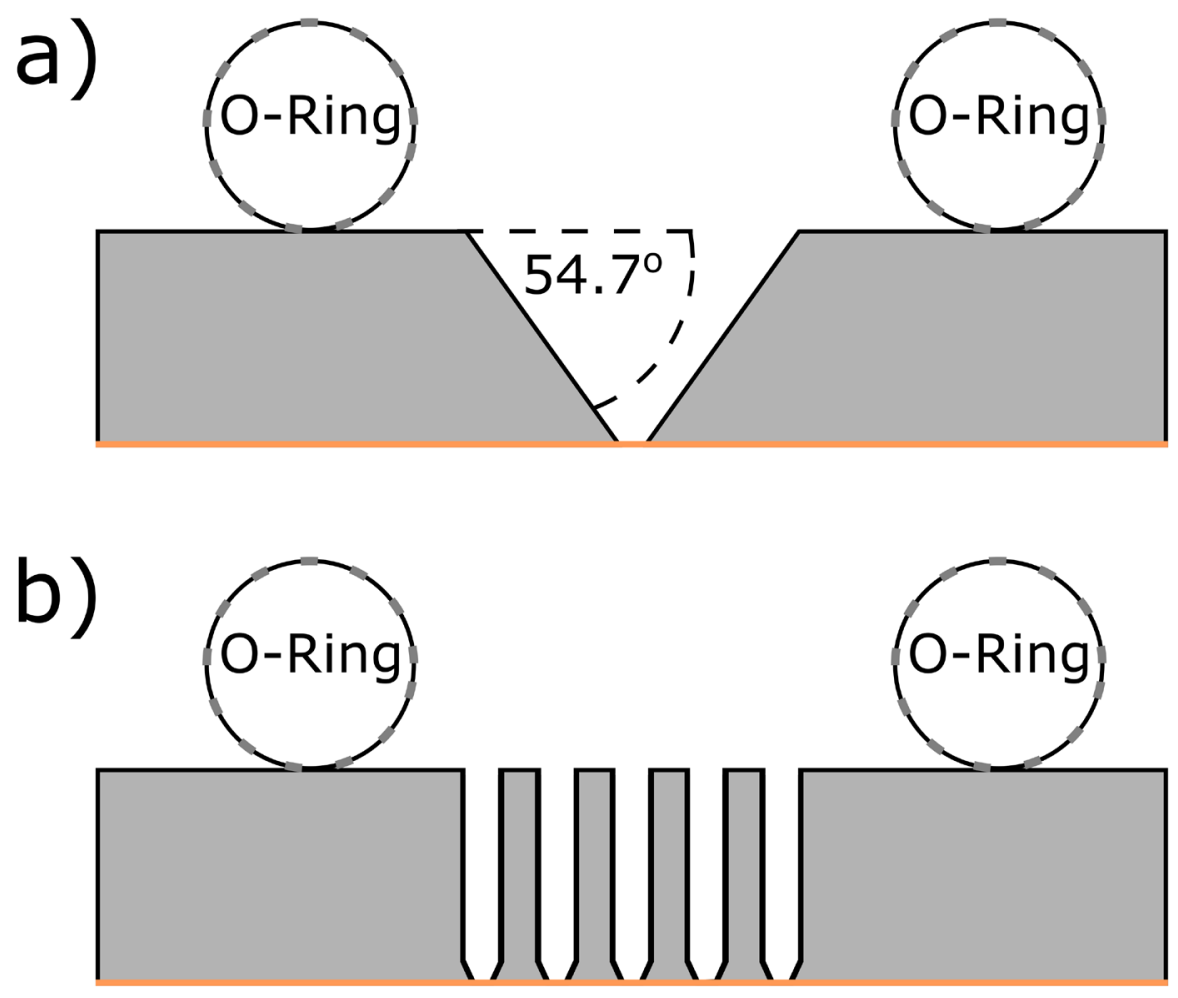

Figure 5.8: a) Cross sectional illustration of a nanofluidic device fabricated with a conventional KOH etch depicting the inward tapering pits. The sealing o-rings dictate a single window for these devices. b) Cross sectional illustration of a multiwindow nanofluidic device fabricated with a DRIE and KOH etch to achieve nearly vertical side walls. Up to five windows can be fit within the sealing o-ring with this strategy.

ring, also shown in the cross section. Figure $5.8 \mathrm{~b}$ shows the cross section for a device utilizing a high aspect ratio anisotropic DRIE etch, detailing nearly vertical side walls for window formation. In this way multiple etching regions and resultant windows can be fit within the constraints of the sealing o-ring. Some optimization however is required in order to perform this etching through the full thickness of the silicon wafer in order to prevent excessive damage to the wafer or the instrument. 


\subsubsection{DRIE Optimization}

A limitation of using DRIE for etching the Si substrate to form free standing silicon nitride membranes is that the plasma does not have etch selectivity for the silicon nitride. As a result, both the top surface must be masked, to limit etching to only the window regions, and the etch must be stopped $10-20 \mu \mathrm{m}$ from the silicon nitride on the opposite surface and the windows finished with a short $\mathrm{KOH}$ etch in order to prevent over etching of the silicon nitride. The etching variability is a concern, as the amount of silicon milled with a single Bosch cycle can be variable to microns. Furthermore, the depth achieved from a single cycle can change across the lateral surface of a standard 4-inch wafer due to nonuniformities in the plasma density generated by the instrument.

\subsubsection{Lateral Etch Variation}

In order to characterize the difference in etch depth as a function of wafer position a bare silicon wafer was patterned with the design for the Hummingbird five window devices using a DRIE specific photoresist (AZ P4620). 200 Bosch cycles were performed and the depth was measured at various positions across the wafer shown in Figure 5.9a using optical profilometry. Figure 5.9b shows a box and whiskers plot for the average etch depth depending on the radial distance from the center of the wafer. As shown in Figure 5.9b, the deepest etching occurs at the center of the wafer, indicating that measurements taken to estimating the etch depth of the window regions should be taken in the center of the wafer in order to prevent over etching of the wafer. 

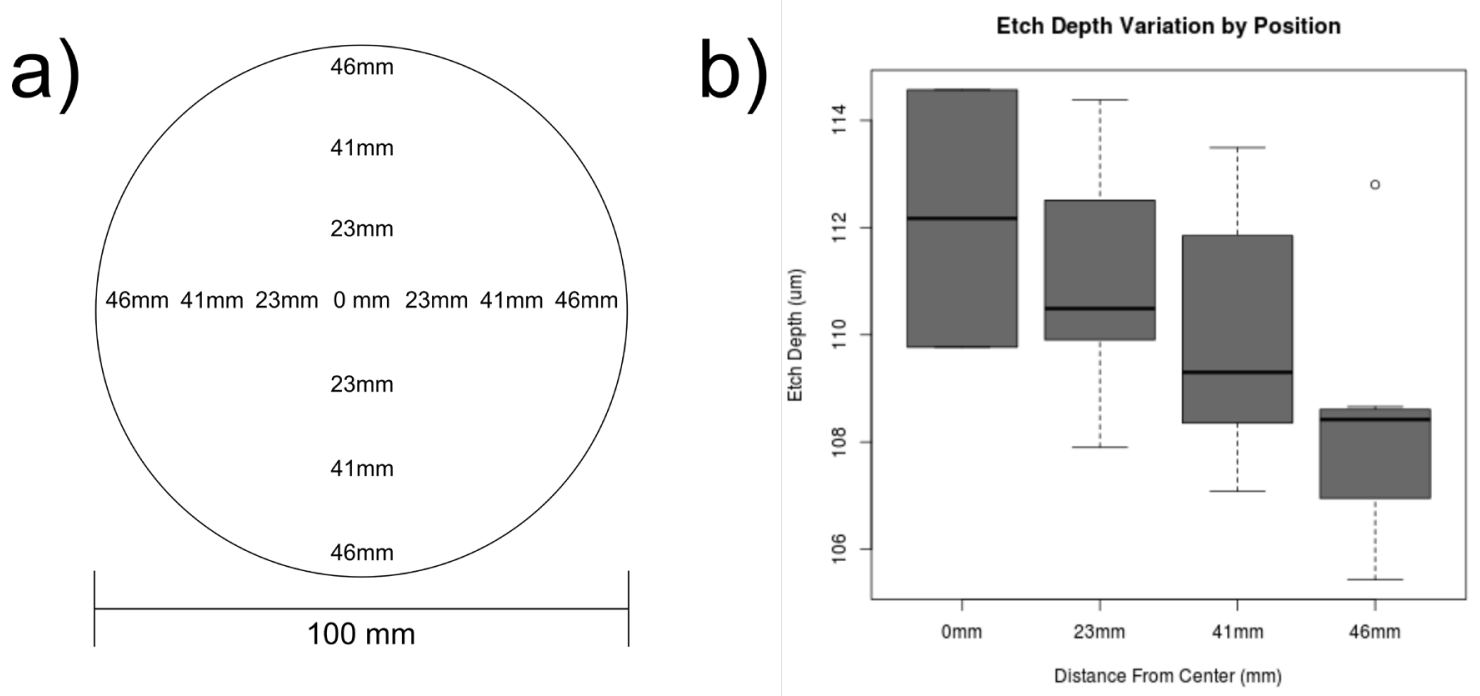

Figure 5.9: a) Depiction of depth measurements taking with optical profilometry across a four-inch wafer. b) DRIE etch depth as a function of position as measured by optical profilometry.

\subsubsection{Resist Thickness Optimization}

Masking for DRIE etching is accomplished using the photoresist AZ P4620 designed specifically for dry etching. While it has good etch selectivity, it is still slowly etched by the plasma during the etch process. To etch through the full thickness of the silicon

Table 5.1: Photoresist conditions for different thickness substrates

\begin{tabular}{|c|c|c|c|c|c|c|c|}
\hline Device & $\begin{array}{c}\text { Spin Speed } \\
(\mathrm{rpm})\end{array}$ & Spin \# & $\begin{array}{c}\text { Soft Bake } 1 \\
(\mathrm{~min})\end{array}$ & $\begin{array}{c}\text { Soft Bake } 2 \\
(\mathrm{~min})\end{array}$ & $\begin{array}{c}\text { Rehydration } \\
\text { Time (min) }\end{array}$ & $\begin{array}{c}\text { Exposure } \\
\text { Time (s) }\end{array}$ & $\begin{array}{c}\text { Depth } \\
(\mu \mathrm{m})\end{array}$ \\
\hline Hummingbird & 1500 & 1 & $\begin{array}{c}70^{\circ} \mathrm{C}: 2 \\
110^{\circ} \mathrm{C}: 3\end{array}$ & $x$ & 30 & 30 & 260 \\
\hline Protochips & 3000 & 2 & $\begin{array}{c}70^{\circ} \mathrm{C}: 1 \\
110^{\circ} \mathrm{C}: 1\end{array}$ & $\begin{array}{c}70^{\circ} \mathrm{C}: 2 \\
110^{\circ} \mathrm{C}: 3\end{array}$ & 30 & 30 & 324 \\
\hline $\begin{array}{c}\text { Dens } \\
\text { Solutions }\end{array}$ & 2000 & 2 & $\begin{array}{c}70^{\circ} \mathrm{C}: 1 \\
110^{\circ} \mathrm{C}: 1\end{array}$ & $\begin{array}{l}70^{\circ} \mathrm{C}: 2 \\
110^{\circ} \mathrm{C}: 3\end{array}$ & 60 & 30 & 428 \\
\hline
\end{tabular}


wafer the resist layer must be thick enough to not etch through during the etch process. The thickness of the wafers for Hummingbird, Protochips, and Dens Solutions devices are $200 \mu \mathrm{m}, 300 \mu \mathrm{m}$, and $400 \mu \mathrm{m}$ respectively. The thickness of the resist then can be optimized such that it survives the etch process for the duration necessary. By optimizing the spin coating speed and number of photoresist layers spun onto the wafer photoresist conditions for each device were determined. Table 5.1 shows the spin conditions, soft bake, exposure, and subsequent maximum etch depth in order to produce devices of the correct thickness for the corresponding holders. For a starting etch region $40 \mu \mathrm{m}$ in width as described in sections 5.3, the etch depth should be taken to within $10-20 \mu \mathrm{m}$ of the full thickness of the wafer. Therefore, the $200 \mu \mathrm{m}$ Hummingbird devices should be etched to $\sim 190 \mu \mathrm{m}$, the $300 \mu \mathrm{m}$ Protochips devices should be etched to $\sim 290 \mu \mathrm{m}$, and the Dens Solutions devices should be etched to $\sim 390 \mu \mathrm{m}$ prior to the final $\mathrm{KOH}$ step.

\subsubsection{Fabrication Protocol}

For any of the commercial LC-TEM holders being used the corresponding mask design from 5.3 is copied across a region to cover a standard 4-inch silicon wafer, in the same way that was described in Chapter 4.3 with the single window devices. Masks are designed as positive masks for use in patterning the etching mask. Wafers are purchased double side pre-coated with LPCVD low stress silicon nitride films $10-50 \mathrm{~nm}$ in thickness. Low stress nitride is critical as standard silicon nitride films have residual stress that will cause wrinkling of the 
membranes after etching is completed. Wafers are first coated with HDMS, used as an adhesion layer for the masking photoresist, at $3000 \mathrm{rpm}$ for $30 \mathrm{~s}$. AZ P4620 is then spun on the same side of the wafer as described in section 5.4.1 depending on the thickness of the wafer. In the case of wafers $300 \mu \mathrm{m}$ in thickness for use with Protochips holders, the initial layer of AZ P4620 is spun at $2000 \mathrm{rpm}$, followed by a soft bake for $1 \mathrm{~min}$ at $70^{\circ} \mathrm{C}$ and $1 \mathrm{~min}$ at $110^{\circ} \mathrm{C}$. A second layer is then spun at $2000 \mathrm{rpm}$ followed by a second soft bake for $2 \mathrm{~min}$ at $70^{\circ} \mathrm{C}$ and $3 \mathrm{~min}$ at $110^{\circ} \mathrm{C}$. The wafer is rested at room temperature for 30 minutes to allow for rehydration of the photoresist prior to developing. The mask for the corresponding design is used in conjunction with a mask aligner and the wafer is exposed for $30 \mathrm{~s}$, followed by developing in $A Z 400 \mathrm{~K}$ in water at a ratio of 1:3. After examining the resist to ensure complete development the wafer is hard baked at $180^{\circ} \mathrm{C}$ for $30 \mathrm{~min}$. During the hard bake, the wafer is not put on the hot plate until it is below $60^{\circ} \mathrm{C}$ and it is not removed from the hot plate after the heat is turned off until it is below $60^{\circ} \mathrm{C}$. This helps prevent cracking of the resist which can occur if the photoresist cools or heats too quickly.

After the hard bake the wafer is ready for DRIE etching. The DRIE Bosh deposition step uses an RF forward power of $10 \mathrm{~W}$, an ICP forward power of $2200 \mathrm{~W}, \mathrm{SF} 6$ at $1 \mathrm{sccm}, \mathrm{C} 4 \mathrm{~F} 8$ at $80 \mathrm{sccm}$, and lasts $5 \mathrm{~s}$. A helium backing of 5 sccm is used for wafers $200-300 \mu \mathrm{m}$ in thickness, while a flow rate of $10 \mathrm{sccm}$ is used for wafers $400 \mu \mathrm{m}$ or thicker. The DRIE Bosh etch step uses an RF forward power of $40 \mathrm{~W}$, an ICP forward power of $2200 \mathrm{~W}, \mathrm{SF} 6$ at $160 \mathrm{sccm}, \mathrm{C} 4 \mathrm{~F} 8$ at 1 
sccm, and lasts $7 \mathrm{~s}$. Bosh deposition and etch steps are alternated where one Bosch cycle contains one deposition step followed by one etch step. In order to prevent over etching of the target thickness, specified at the end of section 5.4.1, the wafer is etched incrementally and measured using optical profilometry after every round of etching to check the depth of the wafer. For a $300 \mu \mathrm{m}$ thickness wafer for making devices for the Protochips holder the target depth is $\sim 290 \mu \mathrm{m}$. Typically, the first 250 cycles reach $132 \mu \mathrm{m}$ depth, the second 250 cycles reach $237 \mu \mathrm{m}$ depth, the next 75 cycles reach $272 \mu \mathrm{m}$ depth, and a final 35 cycles reaches $289 \mu \mathrm{m}$ depth. Each time the etching is performed the cycles are run in this stepwise fashion ensuring that the final depth is near the appropriate value for the thickness wafer being used.

After DRIE etching the remaining $10-20 \mu \mathrm{m}$ of silicon must be removed to form the free standing membranes using $\mathrm{KOH}$ etching. The remaining AZ P4620 on the wafer is stripped by soaking the wafer in nanostrip $2 x$ at $110^{\circ} \mathrm{C}$ until the resist has been totally removed. $\mathrm{KOH}$ is heated slowly with stirring to $80^{\circ} \mathrm{C}$, and the wafer is placed in the $\mathrm{KOH}$ bath at temperature, etching of the silicon is visible by bubble formation caused by the evolution of hydrogen gas as a byproduct of the etching. At around 20 minutes in the $\mathrm{KOH}$ bath the windows may begin to become transparent, shining a light through the backside of the wafer while in the $\mathrm{KOH}$ bath can help determine that the etching is complete. Typically, windows will be fully formed after 30 minutes of etching, and care should be taken to not over etch as the [111] Si planes still etch but at a reduced rate. Over etching of 
a)

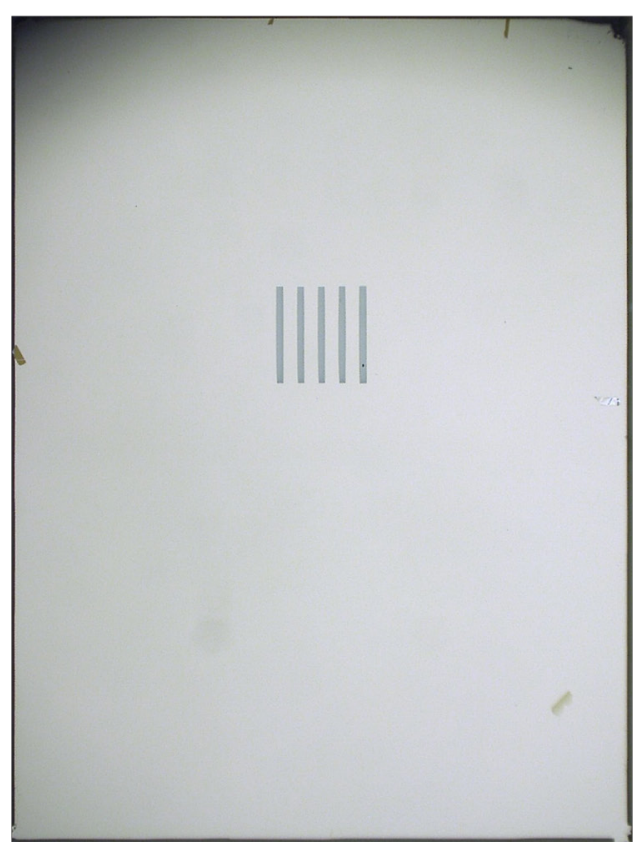

b)

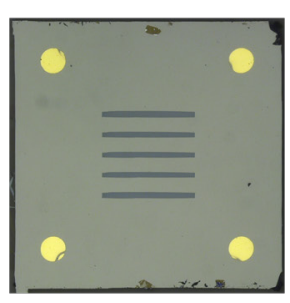

c)

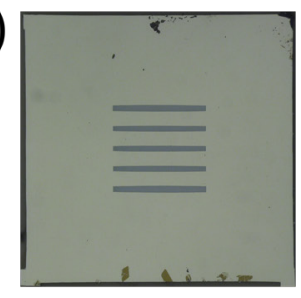

d)

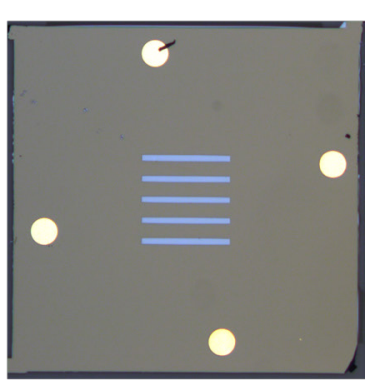

e)

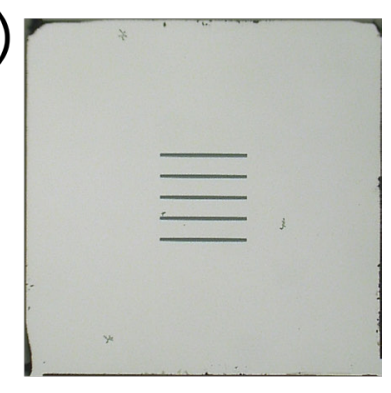

Figure 5.10: a) Large upper device for use in a Protochips Liquid Stage holder. b) Small, lower device for use in a Protochips Liquid Stage holder patterned with spacers. c) Same as (b) but with no spacers. d) Devices for use in a Hummingbird Liquid Stage holder with patterned spacers. e) Same as (d) but with no spacers. Scale bar is $3 \mathrm{~mm}$.

the wafer can cause it to fall apart inside of the $\mathrm{KOH}$ bath and substantially reduces throughput of the process. After the wafer is carefully removed from the bath it should be rinsed in distilled deionized water several times and can optionally be treated with a concentrated $\mathrm{HCl}(5 \mathrm{M})$ soak for 5-10 minutes to remove any metal nanoparticle contaminants. Figure 5.10 shows completed multi-window devices for use with the Protochips and Hummingbird holders fabricated using the above described protocol.

\subsubsection{Spacers and Focus Bars}

Patterning of metal thin films on the surface of the devices prior to window formation is also possible using lift off deposition techniques. Material to act as 
spacers to dictate the minimum thickness of the liquid layer may be patterned on the wafer surface outside the window area, in addition to structures patterned directly on the windows to serve as focusing aids. Electrodes and cell traps are other features which may be useful to pattern on the surface of the windows depending on the application of the devices. Masks for these features are generally negative masks in order to accomplish a negative resist profile, which is advantageous for liftoff deposition techniques. Prior to etching, the desired wafer is coated with AZ 5214E at $3000 \mathrm{rpm}$ for 60 s. Converse to the protocol described in 5.4.2, HDMS is not used as an adhesion layer for liftoff deposition in order to enhance liftoff of the patterned features. The coated AZ 5214E is soft baked at $90^{\circ} \mathrm{C}$ for 2 minutes, followed by exposing the desired mask in a mask aligner for $2 \mathrm{~s}$. At this point, the photoresist is a positive profile and if developed would turn out as such. The wafer is then baked again at $120^{\circ} \mathrm{C}$ in order to reverse the photoresist to a negative profile. The wafer is left to rest at room temperature for 10 minutes for rehydration followed by a $30 \mathrm{~s}$ flood exposure in the mask aligner with no mask present. The wafer is then developed in AZ 340 in water at a 1:5 ratio for $30-45 \mathrm{~s}$. The resist can be easily overdeveloped for fine features and care should be made to optimize the development time based on the features being patterned.

After photoresist patterning the wafer is loaded into a magnetron deposition unit. For patterning gold thin films the chamber is pumped to a backing pressure of $10^{-}$ 7 mtorr, followed by an initial deposition of $10 \mathrm{~nm}$ of chromium as an adhesion 
layer. Chromium is deposited at 2 mtorr and 50 W DC following a RF cleaning step to remove any oxide layer formed on the surface of the target. Deposition time will depend on the tooling factor of the instrument, and for the devices described here was 50 s to achieve films $10 \mathrm{~nm}$ in thickness. Immediately after gold was deposited at 5 mtorr chamber pressure, $50 \mathrm{~W}$ DC, following an RF cleaning step. The deposition rate was $0.5 \mathrm{~nm} / \mathrm{s}$, and films ranging from $50-$ 500nm were deposited depending on application. Immediately after deposition the wafers are removed from the deposition unit and placed into an acetone bath with sonication to begin the liftoff of the photoresist. After 10-15 minutes of sonication the photoresist should be removed leaving behind the thin film deposited on regions not covered by the photoresist. Care should be taken at this point to prevent redeposition of the lifted off thin film back onto the wafer. When removed from the acetone bath the wafer should be immediately rinsed with acetone, followed by distilled water, and then into a nanostrip $2 x$ bath before the wafer can dry. After $10-20$ min of soaking in the nanostrip bath at $110^{\circ} \mathrm{C}$ the wafer can be removed and dried. Examples of patterned thin films are shown in Figure 5.10b, 5.10d, and 5.10e depicting spacers and focus grid bars patterned using the protocol described above. Window patterning, etching, and formation can now be carried out as described in section 5.4.2.

\subsection{Low Dose Imaging Regime for LC-TEM}

The patterned focus bars described in section 5.4 .3 are designed to be used as high contrast fiducials for finding optimal focus before collecting LC-TEM data 

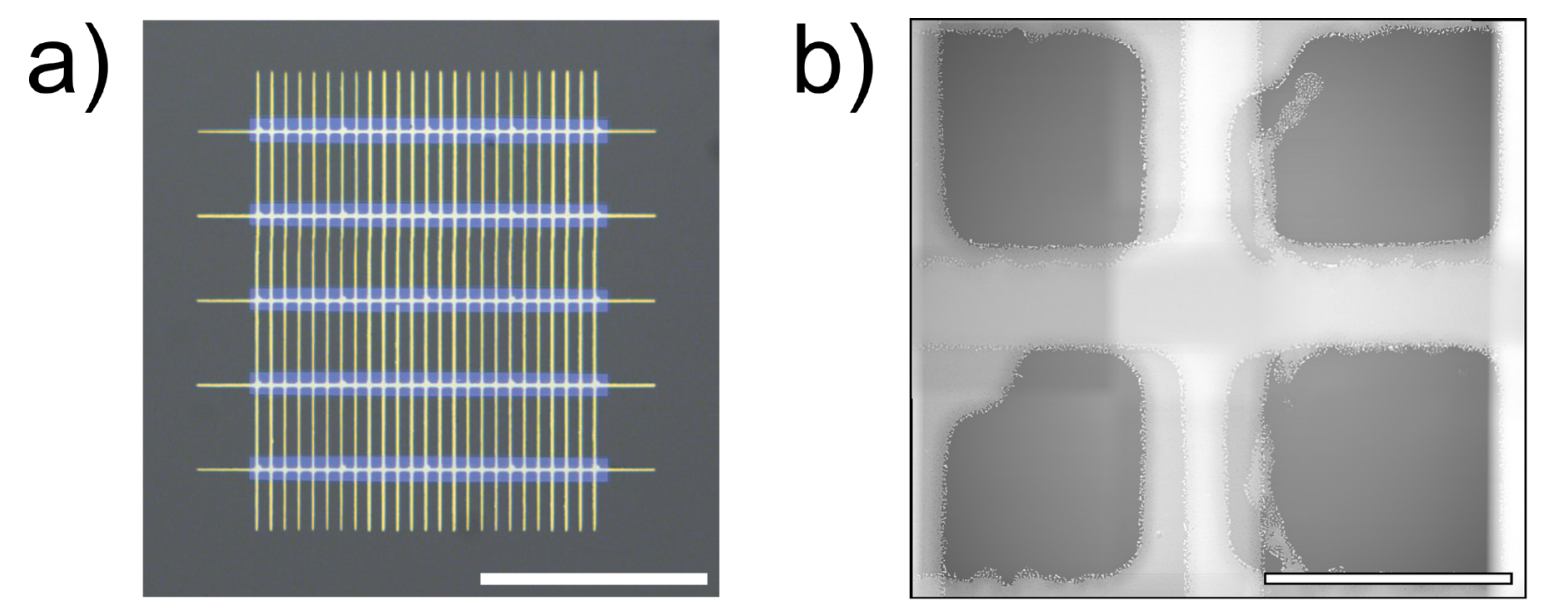

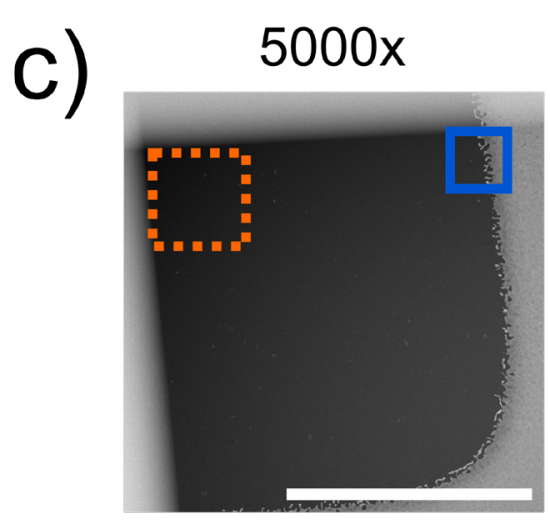

Search

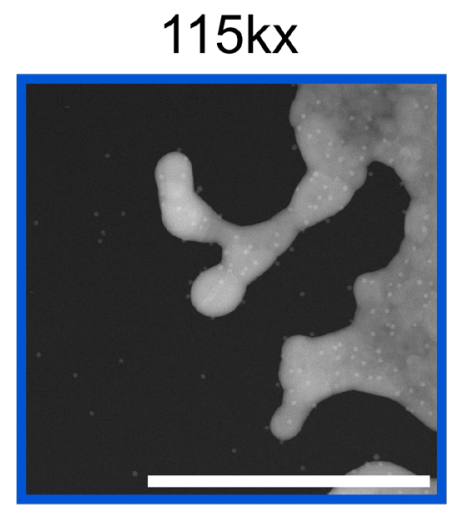

Focus

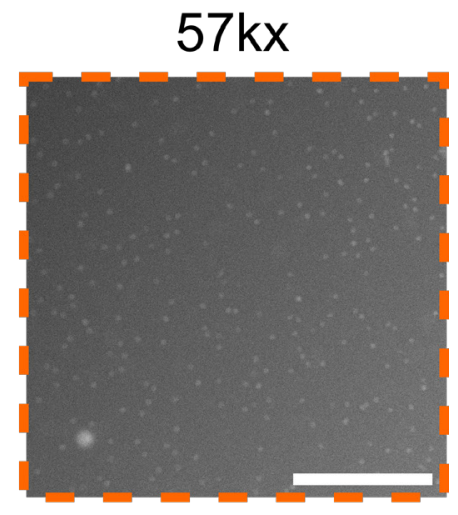

Acquire

Figure 5.11: a) Optical image of multiwindow device patterned with grid bars. b) In-situ image of grid bars assembled within a liquid cell. c) Demonstration of "low dose" imaging of $10 \mathrm{~nm}$ gold nanoparticles depicting the "search", "focus", and "acquire" steps to minimize electron irradiation of the region of interest prior to imaging. Scale bars are $500 \mu \mathrm{m}, 20 \mu \mathrm{m}, 10 \mu \mathrm{m}, 500 \mathrm{~nm}$, and $500 \mathrm{~nm}$ respectively.

sets. For samples which are sensitive to electron irradiation, either for damage events or initiation of particle nucleation, the electron dose on the sample prior to data collection must be kept minimal to avoid aberrant time zero results. While this is well characterized for cryo-EM $(220,221)$, it is considerably more difficult for LC-TEM experiments. Primarily this is due to the lack of features available for focusing and for LC-STEM imaging is exacerbated by the narrow depth of focus caused by the high convergence angles used to form STEM probes. Figure 5.11 
depicts the structure of and use of patterned grid bars in a low dose imaging regime. An optical image of a 5 window device with grid bars is shown in Figure 5.11a, where grid bars are shown bisecting the center of each window on the device. A single imaging window formed by assembling two devices in a liquid stage holder is shown in Figure 5.11b. The high contrast grid bars are clearly visible against the liquid background and the edges display roughness which can be used for precise focusing during imaging. A demonstration of low dose imaging is shown in Figure 5.11c, depicting the "search", "focus", and "exposure" modes used for minimizing electron dose on a sample prior to collecting an image. "Search" is typically low magnification, high refresh rate, and minimal electron flux and is used to find a region of interest for image acquisition (outlined by the dashed orange box). Prior to exposure, the beam is centered over a nearby grid bar for focusing (blue box) and the beam is blanked and magnification is increased. In the "focus" mode, precise focus is found on the grid bars at high magnification, such that the region of interest containing the sample to be imaged is not irradiated by the electron beam in this step. After focus is found, the beam is then blanked again and magnification is reduced back to the "search" magnification. The beam is then centered over the region on interest (orange box), and the beam is once again blanked as imaging settings are changed to the optimal "exposure" settings. The beam is then unblanked and a single, time zero image with minimal prior electron irradiation is collected near optimal focus. The final image shown in the "exposure" mode is of $10 \mathrm{~nm}$ gold 
nanoparticles that are adhered to the same window that the grid bars are patterned on.

While an effective imaging method to reduce irradiation on the sample prior to image collection, it should be noted that there are still several limitations reducing the precision of these grid bars. The membrane bulging effects described in 5.2.1 result in an effective change in eucentric focus for the membrane surface which changes depending on the $\mathrm{x}$ and $\mathrm{y}$ position that focus is found at. Manual focusing thus still results in some small variations between the optimal focus found at the "focus" position and the optimal focus at the "exposure" position. The possibility for automated focusing software to perform surface modeling by finding optimal focus at several points along the grid bars and determining the most likely focus value for the exposure region would provide more accurate imaging and vastly improve the throughput of the technique. It should also be noted that optimally the grid bars be located on the upper window and the sample of interest be near or at the upper membrane. This ensures that the focus plane found by focusing on the grid bars is on the same plane as the sample of interest, and reduces image artifacts caused by beam broadening effects of thick samples (219).

\subsection{Electron Irradiation History}

The dynamic, liquid environment of the liquid cell introduces unknown variables related to the production of radial species and their damage of the sample of 
interest as a result of electron irradiation. A significant number of recent publications in the field have been aimed towards understanding the role of electron-liquid interactions (102), although the effect of large cumulative electron fluxes and its effect on serial experiment acquisition was not characterized before the work described here.

\subsubsection{Lateral Diffusion of Radiolysis Products}

While advantageous, the increased imaging area empowered by the multi window devices described in section 5.4 raises a fundamental question about the mobility of radical species generated from electron irradiation of water. Numerical simulations of the generation and reaction of radiolysis products under TEM relevant conditions suggest that the most reactive species, aqueous electrons, $\mathrm{OH} \cdot$, and $\mathrm{H} \cdot$ radicals react rapidly with the bulk water solvent just outside the area irradiated by the electron beam $(102,104)$. Although other less reactive species such as hydrogen and oxygen gas can be present in significant concentrations up to microns away from the irradiated region their role and effect on the sample chemistry is not well understood. It is not known how damage products produced in one window may diffuse to an adjacent window and impact the sample in that region prior to imaging. Commonly, damage products are observed outside the area irradiated after imaging has occurred (97). Figure 5.12 illustrates this behavior, where Figure 5.12a shows the first frame of a video of irradiation of a $0.1 \mathrm{mM}$ solution of $\mathrm{AgNO}_{3}$ under an electron flux of $0.51 \mathrm{e}^{-/ / \AA^{2}}$. Particles can be seen beginning to nucleate and precipitate under the reducing 
a)

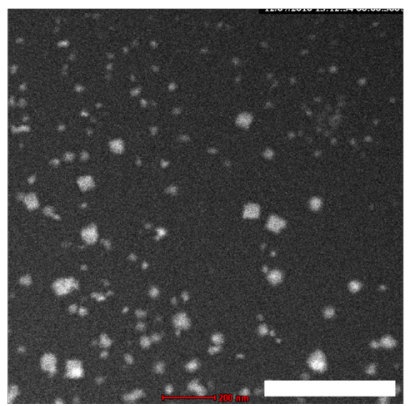

b)

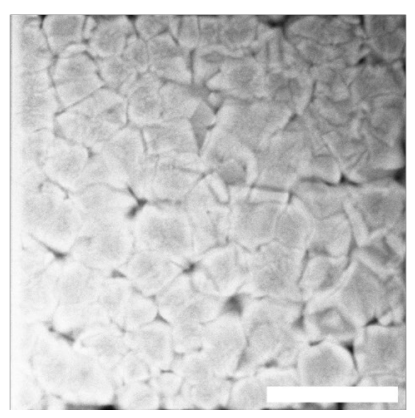

c)

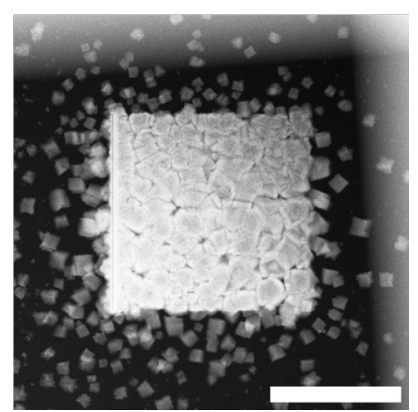

Figure 5.12: a) Initial scan of a STEM experiment precipitating silver nanoparticles from an aqueous precursor. b) Final frame of the same region from (a) where the field of view has not changed. c) Lower magnification image of the region imaged through $(a-b)$ showing the nucleation of particles outside the irradiated region. Scale bars are $400 \mathrm{~nm}(\mathrm{a}-\mathrm{b})$ and $500 \mathrm{~nm}(\mathrm{c})$.

potential of the radiolysis product generated by the electron beam. Importantly, only the area within the field of view in this image is being irradiated by the electron beam. Figure $5.12 \mathrm{~b}$ shows the final frame of the same video, where particles nucleated in the first frame have grown larger over the duration of the video. Figure $5.12 \mathrm{c}$ then shows a wider field of view of the same region immediately after the end of the video. Particles can be seen outside the irradiated region, with the size and number decreasing with increasing distance from the irradiated area. It is unclear if these particles grown outside the imaging area is the result of radiolysis products diffusing from the irradiated region and causing nucleation and growth outside the imaging area or particle nuclei forming within the irradiated region, and diffusing outside of the field of view while continuing to grow. Investigating the role of global cumulative irradiation is necessary in order to be certain that serially acquired data sets from a single device are representative of a sample that has been free of irradiation prior to imaging. 


\subsubsection{Biotin-Streptavidin Crystals}

Since the desired application for our systems is for biological imaging, ideally testing the diffusion of damage products can be evaluated with a biological system. Initially to track the role of increasing cumulative electron irradiation, biotinylated gold nanoparticles were anchored to a streptavidin monolayer through a biotinylated lipid monolayer deposited on the silicon nitride membrane using a Langmuir-Blodgett trough. The gold nanoparticles act as a reporter to a damage even when either the lipid, streptavidin, or biotin molecule is damaged and releases the gold nanoparticle from its anchor on the membrane. By tracking the position of the gold nanoparticles damage events can be tracked over time.

Figure 5.13 shows the results of tracking the positions of particles under electron irradiation for two different areas $(222,223)$. Figures 5.13a and 5.13b maximum displacement of each particle from each frame of the acquired video. As evidence by the displacement of particles between $5.13 \mathrm{a}$ and $5.13 \mathrm{~b}$, the particles in the first video can be seen to have considerably larger displacements than the second video over the same time duration. Figures $5.13 \mathrm{c}$ and $5.13 \mathrm{~d}$ show the $\mathrm{x}$ and y positions of each particle with increasing time, illustrating how the particles in the first video (5.13a and 5.13c) have large displacements and exhibit linear movement throughout the video. Conversely the particles in the second video (Figures 5.13b and 5.13d) have less displacement and stay largely in the same position over the experiment. This indicates that the movement seen in the particles in video 1 is the result of mechanical or electrostatic stage drift that is 
a)

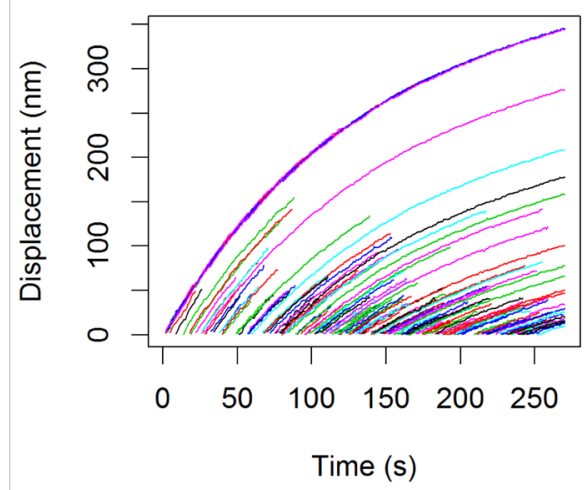

c)

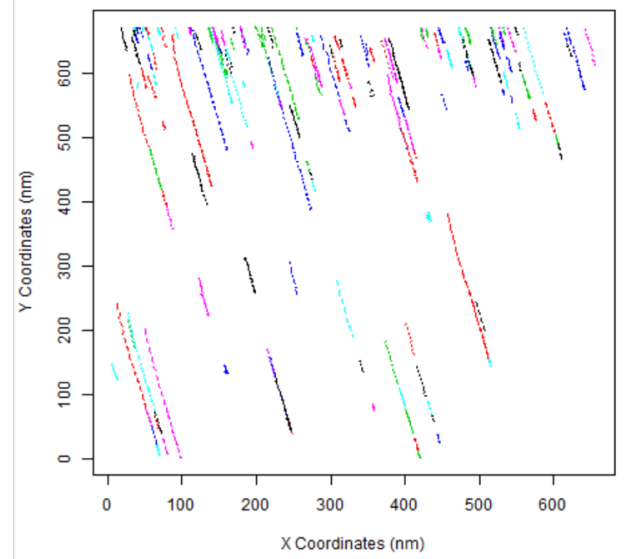

b)

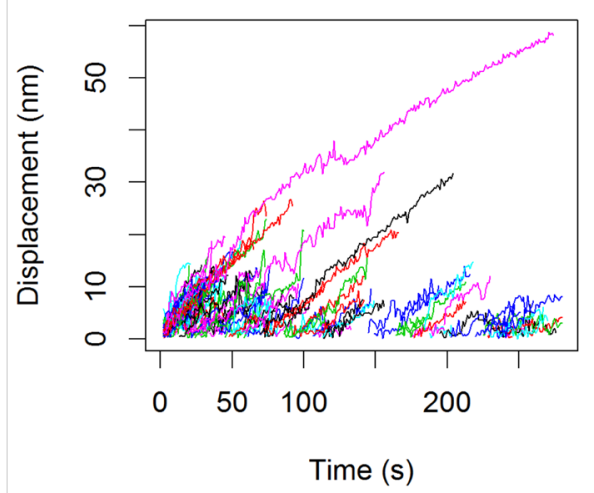

d)

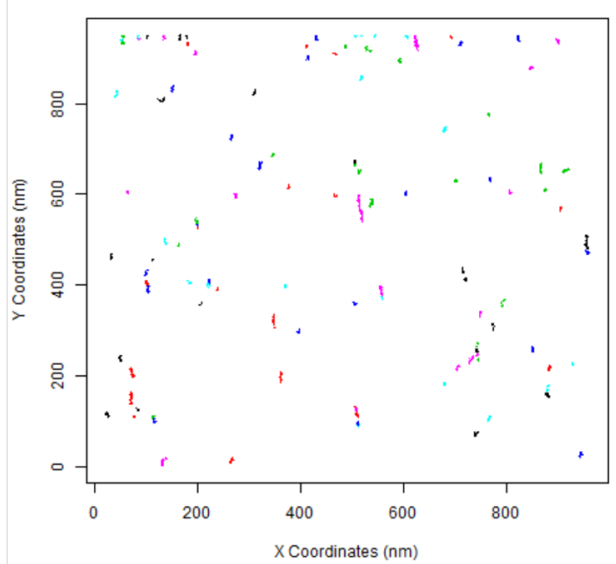

Figure 5.13: a) Particle tracking data from the first video tracking the damage of Au nanoparticles anchored to the window via streptavidin-biotin linkers. b) Particle tracking data from the second video tracking damage, where the displacement of particles is much lower. c) Trajectories of particles tracked from (a), depicting uniform unidirectional motion indicative of mechanical drift. d) Trajectories of particles tracked from (b) depicting random motion.

often seen shortly after loading the sample into the microscope (224). This highlights the necessity for allowing stage drift to settle before data is acquired but also that drift based imaging artifacts should be considered when performing experiments which track the position and location of particles. 

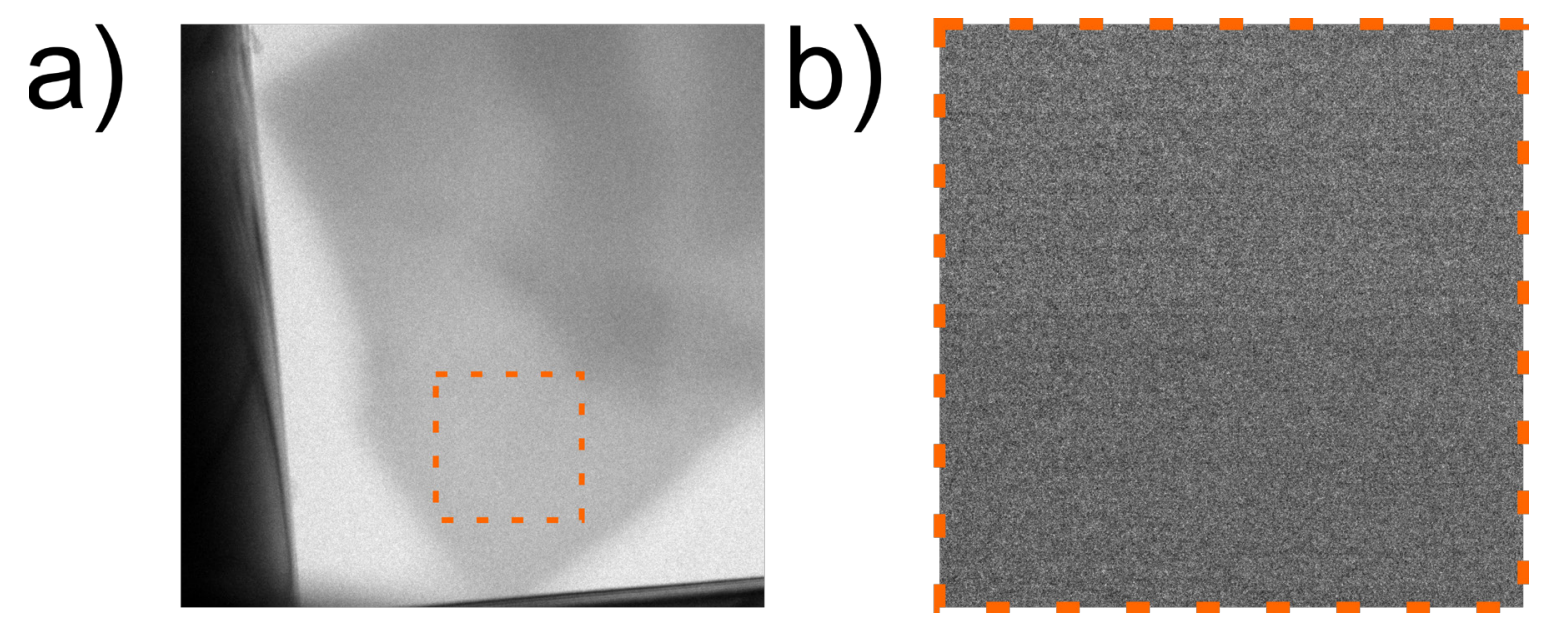

Figure 5.14: a) Low magnification image of 3D catalase crystals in a liquid stage. b) High magnification image of region outlined by orange box in (a) showing high noise and low signal common in very thick samples.

\subsubsection{Catalase Crystals}

Another mechanism for tracking damage over multiple exposures could be tracking the fading of reflections in 2D catalase protein crystals as described for cryo-EM in Chapter 3. Catalase crystals were loaded into the liquid cell and imaging was attempted to collect both diffraction and real space images. Figure 5.14a shows a BF-TEM 2D catalase crystal in liquid, where $5.14 \mathrm{~b}$ shows a magnified region of 5.14a taken with low dose imaging techniques. The liquid thickness proved to be too thick to obtain images with any of the samples loaded, where reflections were not found in the FFT of real space images or in diffraction patterns taken of regions containing crystals. Likely the lack of resolution is due to high thickness of the liquid cell, where stacking of the crystals can result in considerable thicknesses which will dictate the minimum spacing. As such another method was necessary to track damage over time. 


\subsubsection{Silver Nitrate Precipitation}

The precipitation of metal nanoparticles from an aqueous precursor is well characterized and represents the bulk of published material from LC-TEM ( 71 , $97,100,225-228)$. The reduction of aqueous silver nitrate in particular has been well described $(71,98,105)$, where both reaction and diffusion limited growth regimes have been demonstrated depending on precursor concentration and electron flux (98). As such this system presents an ideal way for studying the effects of cumulative electron irradiation on growth kinetics for serially acquired data sets. Figure 5.15 shows data collected from a single multi window device which was loaded with a $0.1 \mathrm{mM} \mathrm{AgNO}_{3}$ precursor. STEM imaging was performed with an electron flux of $0.51 \mathrm{e}^{\mathrm{e} / \AA^{2}}$ for 20 minutes as silver nanoparticles were nucleated and grown on the silicon nitride windows. Figure 5.15a shows a map of the window regions where the red numbers are the location and order of STEM videos which were acquired. Figure $5.15 \mathrm{~b}$ shows frames from different time points of STEM videos at the same time points, corresponding with videos 2,6 , and 12 . The appearance and growth of particles can be seen in these frames, where tracking analysis was performed on each video data set to track the size of the particles over the duration of the video $(222,223)$.

Figure $5.15 \mathrm{c}$ shows the mean particle diameter for each video, where the growth rate of particles in each video follow a $t^{1 / 2}$ power law, indicating reaction limited growth $(229,230)$, independent of the order of acquisition. This indicates that for 
a)

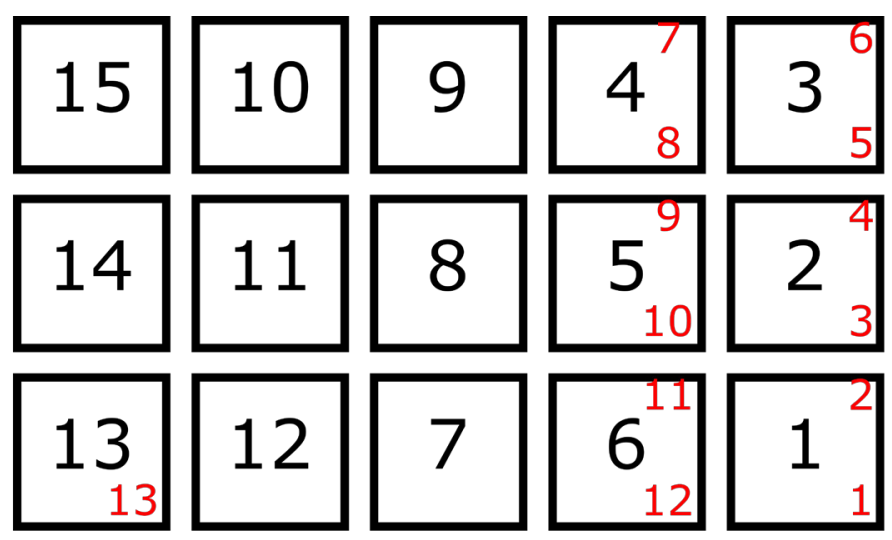

Window Number Video Number
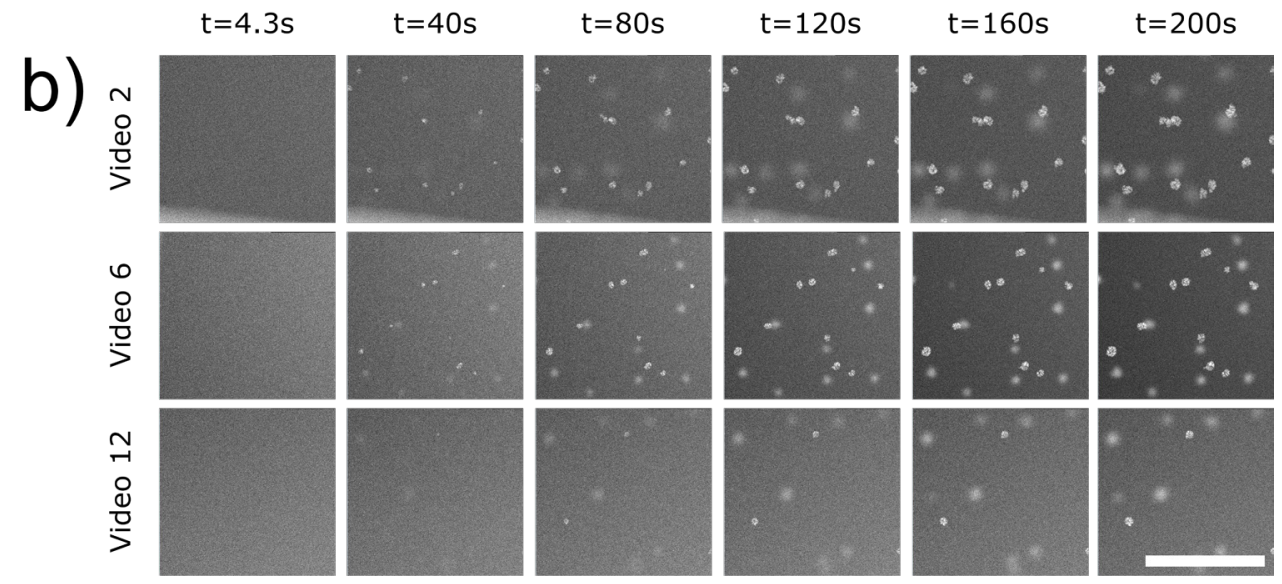

c)

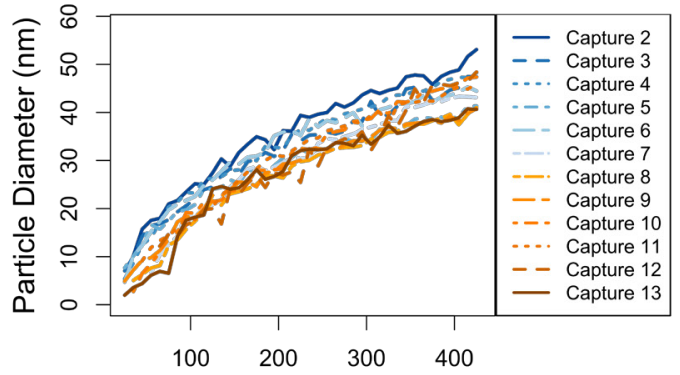

Time (s)

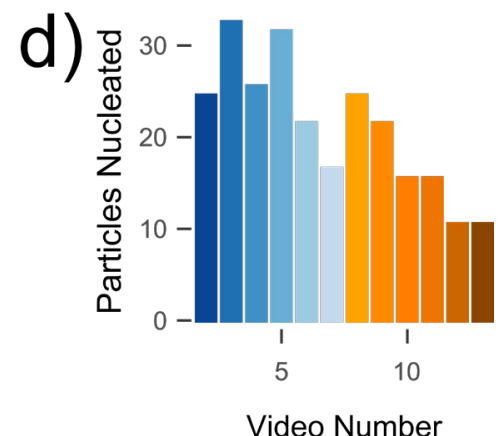

Figure 5.15: a) Window map depicting windows (black numbers) and experiment locations (red numbers). b) Frames of experiments 2, 6, and 12 showing the precipitation and growth of silver nanoparticles from a silver nitrate precursor. c) Plot of mean particle diameter increase over time for each experiment. d) Total number of particles nucleated for each experiment.

at least the distance separating the experiments in these experiments they are far enough apart for the growth kinetics to not be impacted by irradiation products 
from a previous experiment. Importantly, experiments performed closer together or growth kinetics which are diffusion limited may display increased sensitivity to irradiation history but should be determined for each system in order to reduce potential imaging artifacts. One potential artifact observed however is a decrease in nucleation rate with increasing electron irradiation history. This behavior is illustrated by Figure 5.15d, where the number of particles nucleated for each video decreases as a function of increasing cumulative global electron flux. While the holder which was used for these experiments was capable of flow to the imaging area the experiments were performed in a static environment. It was thought that this may be the result of a depletion of the precursor solution, but it was also considered that a changing volume as a result of a wedge-shaped sample and changing thicknesses could also affect nucleation behavior by changing the effective concentration of radiolysis products (231). For the data in Figure 5.15 the thicknesses for each imaging region was unknown and could not be determined for certain if the decrease in nucleation rate was a result of depletion or thickness changes.

Growth experiments were repeated for both $0.1 \mathrm{mM}$ and $0.5 \mathrm{mM} \mathrm{AgNO}_{3}$ precursor solutions both with and without flow of fresh precursor to the imaging area. The thickness of each imaging region was measured with EELS in order to ensure that the thicknesses of each experiment is equivalent. Figure 5.16a and $5.16 \mathrm{~b}$ shows results from a $0.1 \mathrm{mM}$ solution without flow of fresh precursor. Figure $5.16 \mathrm{a}$ is the final frame of each experimental video, while $5.16 \mathrm{~b}$ is the 

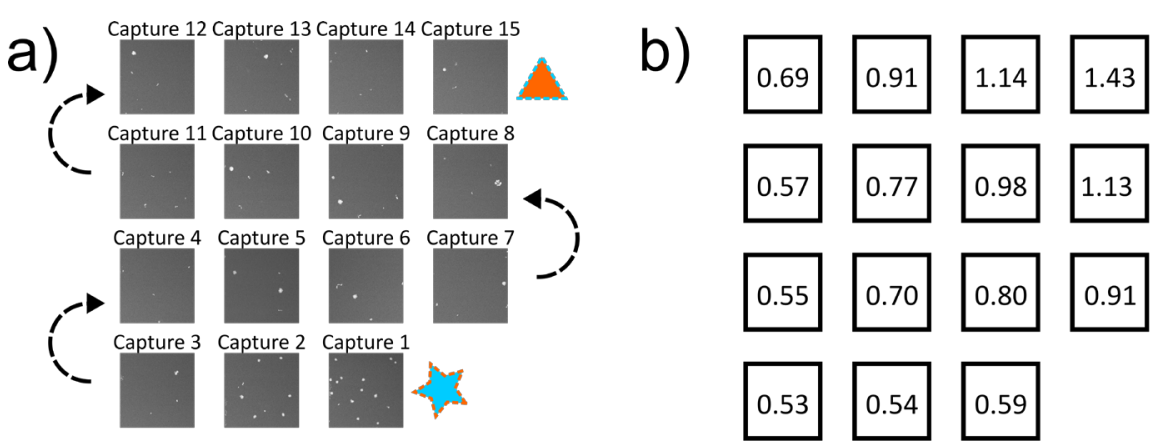

c)

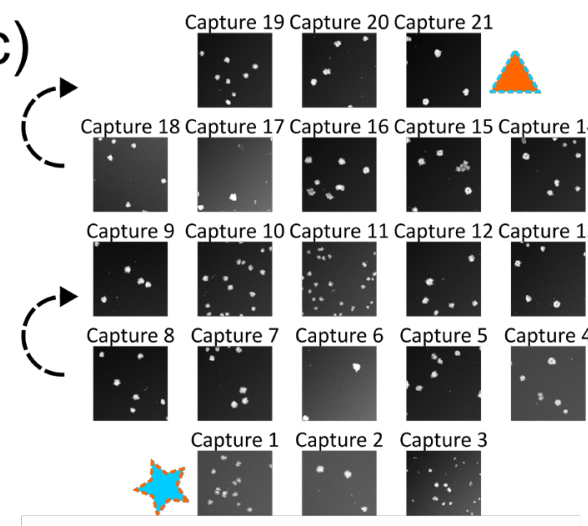

d)

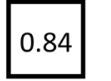

1.17
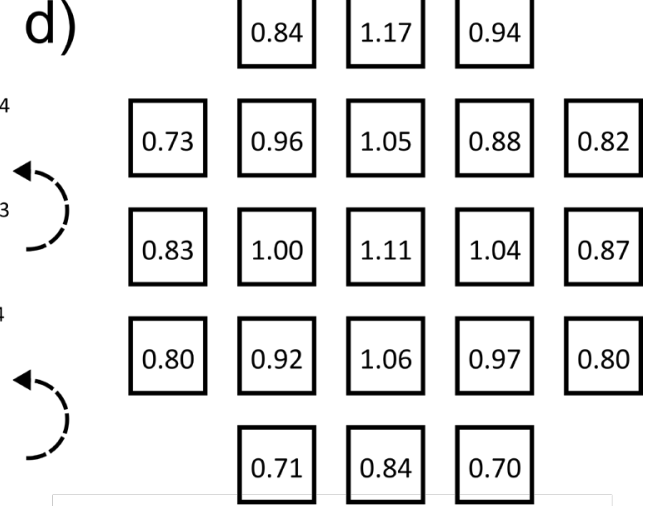

0.97

0.80

e)

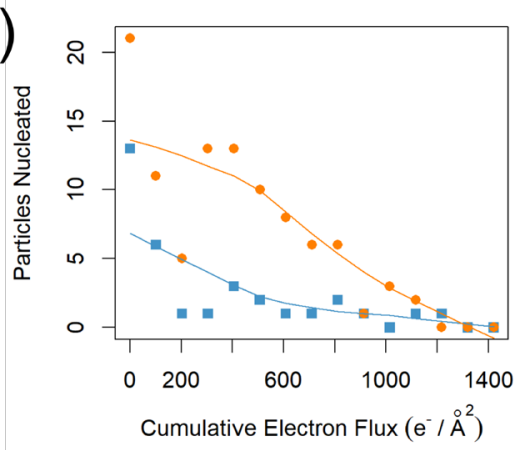

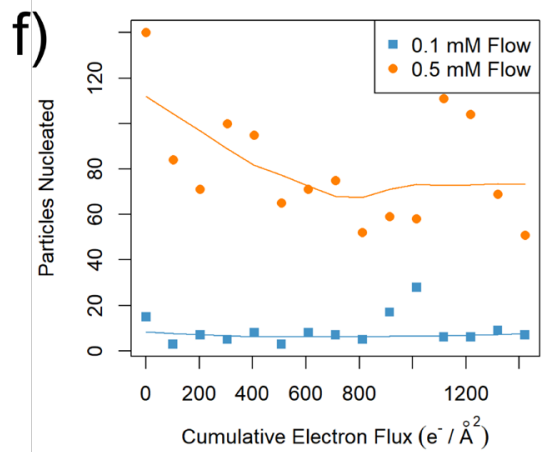

Figure 5.16: a) Final frames of silver nanoparticle growth experiments with no flow where the location of each image represents the window within the grid that experiment was performed at. The blue star notates the first experiment and the orange triangle notates the final experiment. b) Thickness measurements in IMFP for each experiment corresponding to locations in (a). c) Same as (a) but fresh precursor was flowed at a rate of $0.5 \mu \mathrm{L} / \mathrm{min}$. d) Thickness measurements in IMFP for each experiment corresponding to locations in (c). e) Particle counts for experiments without flow for both $0.5 \mathrm{mM}$ (orange) and $0.1 \mathrm{mM}$ (blue) silver nitrate precursor solutions. f) Same as (e) but for experiments with flow of fresh precursor.

thickness in IMFP corresponding to each experiment location from 5.16a. The thicknesses for each imaging window can be seen to be largely equivalent, while visibly the number of particles in the last frame from $5.16 \mathrm{a}$ can be seen to 
decrease with increasing experiment number. Figure 5.16c and 5.16d are experiments performed identically to the ones in $5.16 \mathrm{a}$ and $5.16 \mathrm{~b}$ but during the course of imaging precursor solution was flowed to the imaging area at a rate of $0.5 \mu \mathrm{L} / \mathrm{min}$. Figure $5.16 \mathrm{~d}$ shows effectively equivalent thicknesses for each experiment taken relative to each other and also the data taken in 5.16a-b. For these experiments however, particles continue to nucleate for each experiment regardless of the history of electron irradiation. Figure 5.16e-f show the number of particles nucleated with increasing cumulative electron flux for both 0.1 and $0.5 \mathrm{mM} \mathrm{AgNO}_{3}$ solutions both with and without flow. For each, regimes without flow show a reduction in particle nucleation rate by the final experiment, while regimes with flow show consistent nucleation even until the final experiment. This demonstrates that for some experiments, the total cumulative electron flux can be an important factor in the observed results, necessitating careful characterization of the sample under observation to be sure that observed results are representative of a sample which has not yet received previous irradiation effects. Biological samples are especially susceptible as described in the next section.

\subsection{Imaging Whole Cells with LC-TEM}

Imaging biological structures with LC-TEM additionally needs to take special care to control the history of electron irradiation on the sample as beam damage can drive morphological changes which can be misinterpreted as physiological behavior or morphologies if not careful (193). The focus aids described in 5.5 as 

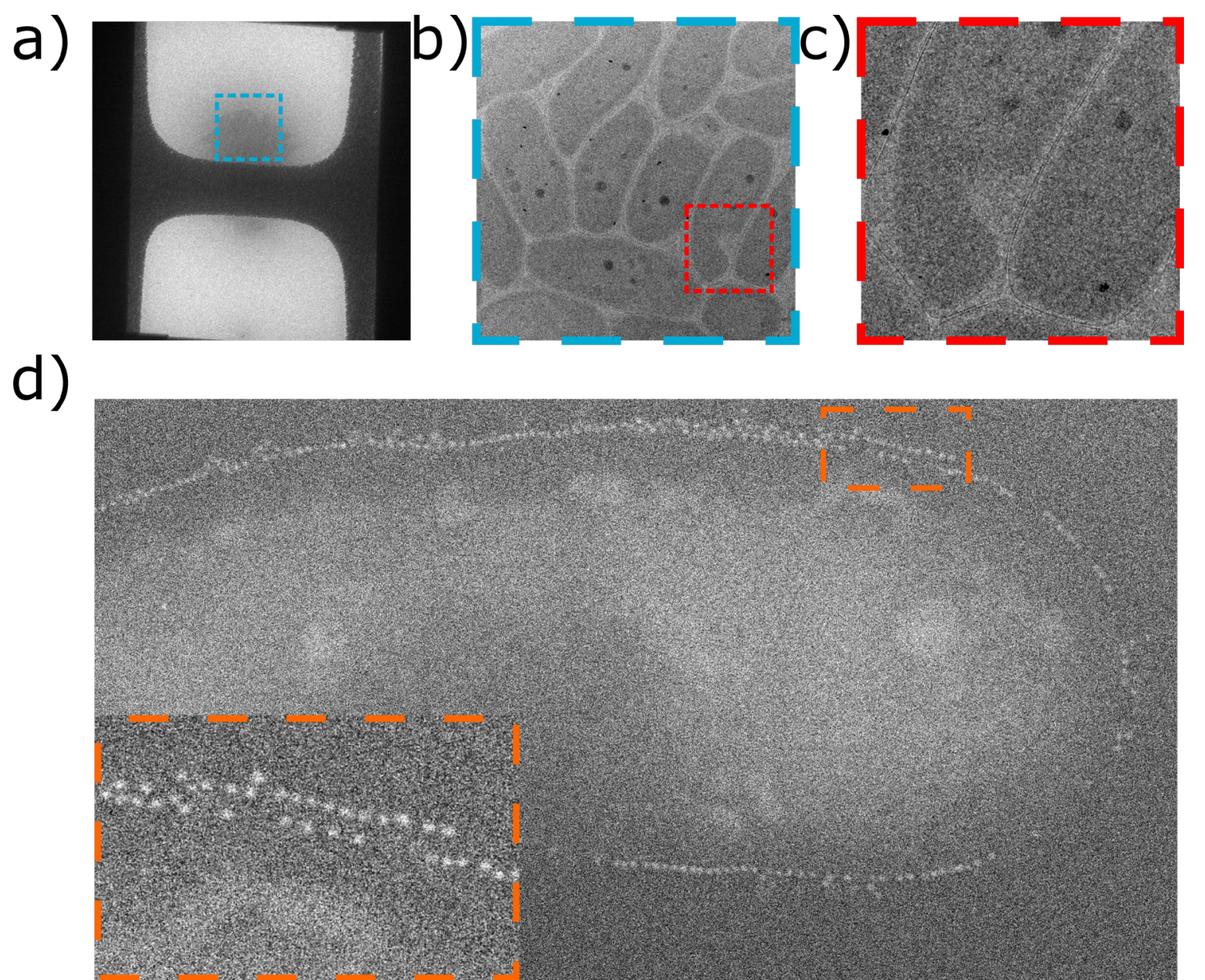

Figure 5.17: a) Low magnification of liquid cell where grid bars are visible with a cluster of cells near the center (outlined in blue). b) Image of region outlined in (a) of C. metallidurans. c) Enlarged region outlined in (b) showing internal details and cell membranes. d) Biotinylated nanoparticle labeling of bacteria in the liquid cell.

especially important for acquiring in focus images of whole cells within minimal irradiation prior to image collection. Figure 5.17 shows images of organisms acquired in LC-TEM using these focus aids in a low dose imaging regime as described in section 5.5. All images were collected at a flux of 0.5 or $1 \mathrm{e}^{-/} / \AA^{2}$ with minimal flux prior to image collection. Figure 5.17a-c are of the organism Cupriavidus metallidurans, where 5.17 a is a low magnification image during the 
"search" mode of low dose imaging. $5.17 \mathrm{~b}$ is an image of $C$. metallidurans cells from the region outlined in $5.17 \mathrm{a}$, while $5.17 \mathrm{c}$ is a cropped section of $5.17 \mathrm{~b}$ highlighting the membranes of the cells and some internal structures. Figure $5.17 \mathrm{~d}$ is a bacterium which has been labeled with $10 \mathrm{~nm}$ biotinylated gold nanoparticles, demonstrating the ability for cellular labeling of structures which may not be resolvable without some kind of labeling aid.

\subsubsection{Electron Damage of Whole Cell Structures}

Beam damage is a critical consideration of electron microscopy of biological samples, where the incident electron beam can break bonds of the sample molecules through primary and secondary damage (193). For biological samples, electron beam-sample interactions are perhaps most well characterized for cryoEM, where artifacts such as resolution loss, particle movement, and bubble formation has been characterized as a function of increasing electron flux (198, 232-234). With liquid samples however, radiolysis and its products can cause further damage with the ability of the generated radical species to react with the sample and cause morphological changes. While the results in section 5.6 suggest that the radical species in one imaging region do not diffuse far enough away to cause damage in an adjacent region, the effects of cumulative electron flux on a single sample have not been well characterized for liquid samples.

To evaluate the effects of electron flux on a biological sample, C. metallidurans was imaged with LC-TEM. Low dose imaging techniques in combination with patterned focus bars described in section 5.5 were used to minimize electron 


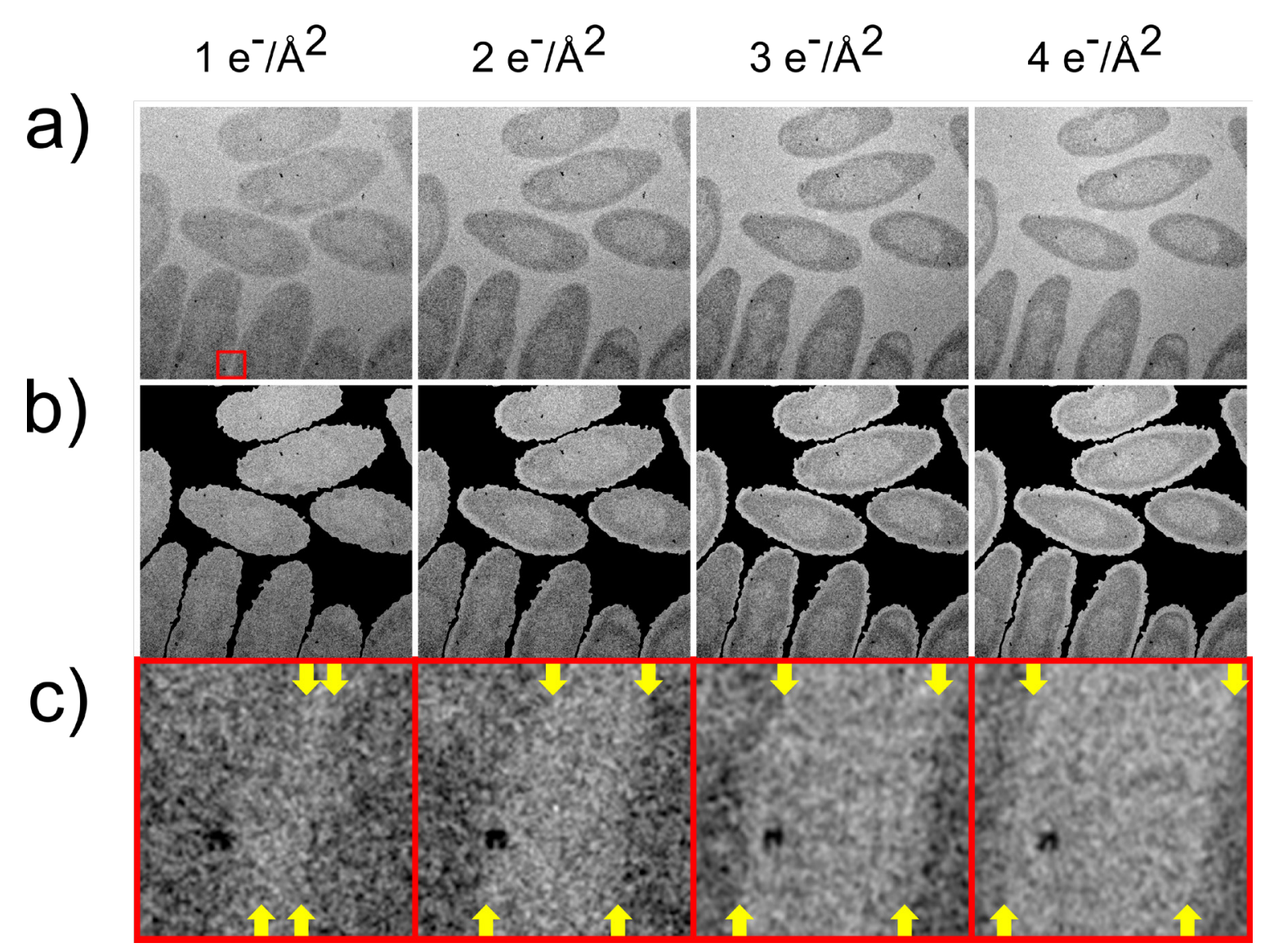

Figure 5.18: a) Damage series of $C$. metallidurans at increments of $1 \mathrm{e}^{-/} \AA^{2}$ for each frame. b) Outline of cells in the first frame of (a) projected over each subsequent image depicting cell shrinking with increasing cumulative electron irradiation. c) Magnified view of region depicted by red box in (a) showing cell membranes withdrawing from a metal nanoparticle on the silicon nitride surface.

irradiation of the cells prior to image collection. Figure 5.18 shows a damage series taken of cells, where the first frame shows a BF-TEM image of cells acquired with an electron flux of $1 \mathrm{e}^{-} / \AA^{2}$. Within this first frame internal structures are clearly visible, and the beam was blanked for 20 minutes with the expectation that some morphological changes or physiological processes may be observable from the internal features that are visible. The next image was acquired with an electron flux of $1 \mathrm{e}^{-/ / \AA^{2}}$ for a total cumulative flux of $2 \mathrm{e}^{-} / \AA^{2}$. This was repeated two more times for a final cumulative flux of $4 \mathrm{e}^{-/} / \AA^{2}$. By the second frame, and even 
more apparent by the $4^{\text {th }}$ frame, cellular changes are visible in the form of the cells shrinking relative to their initial position, which has been reported previously for other cells imaged with LC-TEM (116). 


\section{Chapter 6: Nanofluidic Platform for Directed Flow and Enhanced Environmental Control}

\subsection{Introduction}

The improvements to imaging area described in Chapter 5 were able to solve some issues of reproducibility and increase the sampling capacity of individual LC-TEM experiments. However, inconsistencies in thickness variation between separately assembled devices and the ability for liquid flow to bypass the imaging area continue to constrain the science applications of the technique. While current holder designs may allow for enhanced control over thickness, the strategy of flow for current commercial solutions for LC-TEM do not allow for practical solutions for solving issues related to bypass of flow. I therefore set about redesigning both the nanofluidic holder as well as the nanofluidic devices to overcome these limitations. Chapter 4 highlighted how the design of current commercial holders are limited by available imaging area, thickness reproducibility, and bypass of flow. The issues of constrained imaging area were solved by changing the fabrication protocol as described in Chapter 4. Importantly, those modifications are equally transferable to the new devices that will be described below, although they will not be discussed specifically.

The strategy for solving issues related to thickness reproducibility and bypass of flow is illustrated in Figure 6.1. The strategy for assembling devices with current commercial devices entails manually sandwiching a liquid sample between two devices. This method is sensitive to environmental contaminants or 


\section{a)}

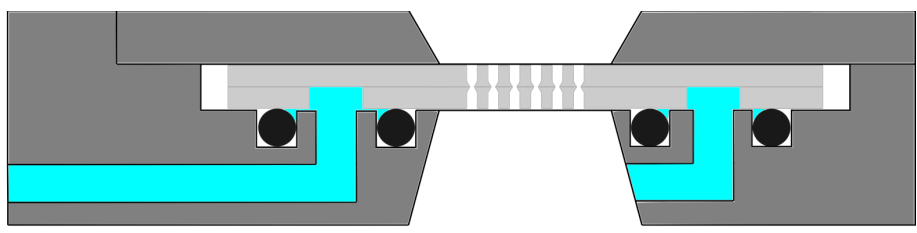

b)

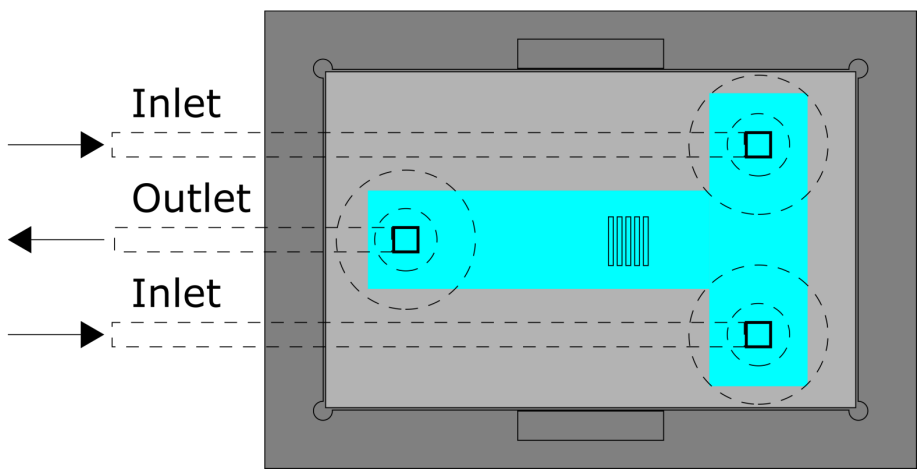

Figure 6.1: a) Cross sectional view of a directed flow imaging strategy depicting the sealing and delivery of flow to the imaging region. Features in this image are to scale. b) Top down view illustration of a directed flow holder demonstrating how liquid sample is forced to flow over the image area minimizing bypass of flow.

nonhomogeneous samples which have a tendency to clump together or sit on top of spacer material that is supposed to dictate the sample thickness. The devices outlined here for the new platform are bonded together during fabrication, both sealing the devices and dictating the channel for fluid flow. This strategy ensures that the thickness of the subsequent liquid layer is precisely dictated by the thickness of the patterned spacers, where cleanliness is ensured by fabrication inside a class 1000 clean room. The holder which holds the devices are designed such that flow occurs directly from the microfluidic lines into the fluid channel within the devices. Figure 6.1 shows this regime, where flow enters the devices through a vertical flow channel etched into the bottom of the devices. Sealing of these channels against the holder is achieved with o-rings, which restrict the 
liquid flow from any direction except for into the vertical flow channel and inside the devices. The bonding and spacer material also acts as a microchannel which forces the liquid sample to flow directly to the imaging area. Multiple windows can be fabricated in these devices, where the lack of a sealing o-ring around the etched windows means that the only constraint for window size is the aperture in the lid and holder, allowing for larger and potentially more windows than the five that has been optimized for the other commercial holders. After flowing across the imaging area, the fluid channel delivers the liquid sample to another vertical flow channel and an outlet microfluidic line. This strategy of flowing sample vertically into a flow channel has been described before, although the published systems only have a single inlet and a single outlet channel (76, 92, 93, 95, 235).

This strategy would not only allow for reliable control over the thickness of the sample, but the directed flow regime would also permit mixing to happen inside the devices rather than outside. Mixing is advertised as a feature for some commercially available holders (125), but the mixing in these holders happens outside the actual devices and far away from viewing area meaning the reaction products can mix and observed results at the imaging area are not true time zero observations. Further, it is not clear what kind of "filtering" effect the devices have on what makes it in between the devices to the imaging area versus what flows around the devices such that the representativeness of the observations made at the imaging area may be questionable. The directed flow strategy outlined below 
was designed to overcome this issue, where mixing reactants do not meet until just before the imaging area.

\subsection{Design of Nanofluidic Holder}

When designing custom holders for FEI and JEOL transmission electron microscopes, the holders can be divided into three segments, each which performs a different function and has different design constraints. The first is the design of the shaft of the holder, which is responsible for triggering and actuating the load lock mechanism for insertion into the microscope column, as well as creating a seal against the wall of the load lock chamber to prevent vacuum loss. The second is the design of the tip of the holder which holds the sample, microfluidic tubing for sample flow, and o-rings for sealing in the case of liquidcell holders. Finally, the body of the holder is the portion which sits entirely outside the microscope, and contains bulkheads and housing for microfluidic tubing, electrodes, as well as being a surface for handling the holder during removal/insertion and sample loading. Especially for the first two components, the tip and shaft, very precise tolerances need to be met in order to fit within the microscope. The sections below will describe how these tolerances are determined, replicated, and manufactured for each component of both FEI and JEOL microscopes. 


\subsubsection{Thermo Fisher/FEl 3 Port}

Holders for Thermo Fisher/FEI microscopes have the highest constraints on the dimensions of the tip due to the design of the microscope pole pieces and goniometer. To determine both the tolerances for replicating the shaft to fit the microscope goniometer multiple holders were measured using a set of electronic calipers. With this method average values of holder diameters, and distances between critical features, such as the tip, the body, the pin, and o-ring were determined so machining could be performed within the tolerances necessary.

\subsubsection{Thermo Fisher/FEl Shaft}

Figure 6.2a illustrates a fully assembled liquid stage holder for use with goniometers found on Thermo Fisher/FEI microscopes. The red arrow in Figure 6.2a points to the base of the shaft, which rests against the outside of the goniometer. The design for the body of the holder should be flush with this surface to allow for the holder to be fully inserted into the microscope.

Proceeding to the right down the length of the holder, the blue arrow highlights the diameter of the shaft which sits just inside the goniometer. The diameter of this region of the holder should be measured closely, as this section of the holder is responsible for triggering two microswitches that sit just inside the goniometer.

One microswitch triggers the load lock cycle during the holder insertion phase, and the other microswitch allows the gun blank to be disengaged once the sample is fully inserted. If the diameter of the shaft is not large enough one or both of these microswitches may not be engaged, preventing use of the holder. 
a)

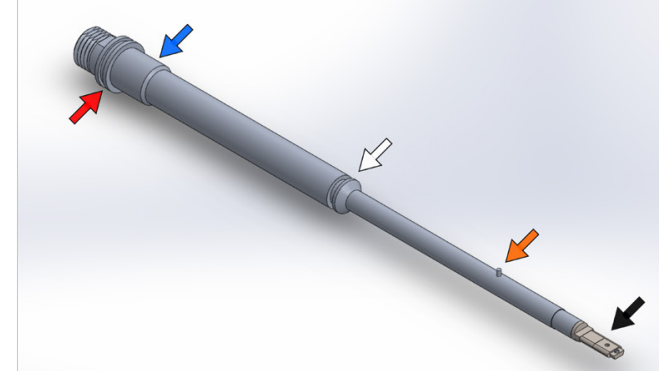

b)

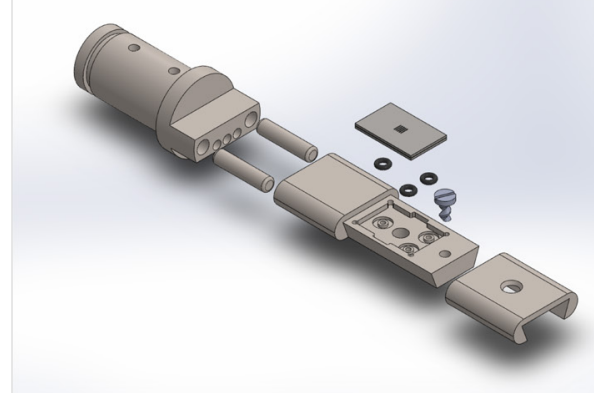

Figure 6.2: a) CAD model of a complete holder for use in a Thermo Fisher/FEI microscope. Critical dimensions are labeled on the shaft on the holder where the base (red arrow), microswitch trigger (blue arrow), shaft o-ring (white arrow), pin (orange arrow), and viewing aperture (black arrow) are depicted. b) Exploded view of 3 port holder showing o-rings, devices, lid and pins for alignment. The two stage assembly of the tip is depicted where microfluidic lines would additionally run through the tip.

The next critical feature is marked by the white arrow and notes the position of the sealing o-ring for the holder. The distance of this o-ring from the base of the holder (marked by the red arrow) is a critical dimension, and the width and depth of the o-ring groove are critical to ensure proper sealing against the vacuum of the instrument. After the o-ring the orange arrow notes a pin that projects vertically from the shaft of the instrument. The location, orientation, and size of this pin is important as it actuates the valve mechanism of the load lock which allows the holder into the microscope during insertion. The distance from this pin to the o-ring and the base of the shaft is important to make sure the holder sits in the correct location when inserted. Additionally, the pin should be parallel to the direction the electron beam is transmitted relative to the orientation of the tip to ensure the tip of the holder is normal to the electron beam. Finally, the end of the holder and the tip is noted by the black arrow. The distance from the electron imaging area in the tip (in this case the aperture around the electron transparent 
membranes) to the base of the shaft is critical to ensure the imaging area sits within the path of the electron beam. If this dimension is too short or too long then the imaging area will not be visible even at the $x$ and $y$ maximums of the goniometer. All dimensions were measured across several known commercially purchased holders and averaged to find the tolerances for each dimension. Measuring manually by hand with electronic calipers proved to be accurate enough to allow for machine of a shaft that met specifications for the Thermo Fisher/FEI microscope goniometers.

\subsubsection{Thermo Fisher/FEI Tip}

Figure $6.2 \mathrm{~b}$ shows an exploded illustration of the liquid tip including the microfabricated devices with the fluidics channel and electron transparent windows, as well as the o-rings used for sealing. The horizontal channels which hold $360 \mu \mathrm{m}$ microfluidic tubing are visible (although the microfluidic tubing is omitted from this illustration), each of which terminate at their respective vertical flow channel and sealing section with o-rings. Because the tolerances of these lines are very small and the length to mill through the tip is far, the tip was designed to be machined in two sections. One the left side of Figure $6.2 \mathrm{~b}$ is the portion of the tip which contains an o-ring groove which seals against the shaft of the tip to maintain hermeticity of the holder. This section also is tapped for screws to ensure the tip stays secure in the end of the shaft and stays square to the shaft pin (orange arrow in Figure 6.2a). To ensure proper alignment of the base of the tip to the second portion alignment pins are used which also provides 
rigidity and support at the joint between the two components. The right portion of the tip in Figure $6.2 \mathrm{~b}$ holds the microfabricated devices which seal with o-rings. The lid (rightmost component of Figure 6.2b) fits onto the tip, where the dovetail design ensures that the rails in the lid compress the devices onto the o-rings for proper sealing. The very end of the tip is tapped for a small screw to secure the lid in place and prevent it from sliding off during operation. The maximum dimensions of the tip were also determined by measuring the largest known commercially available holder tips and ensuring that the dimensions of the tip described here were within those tolerances.

\subsubsection{JEOL 5 Port}

Similar to the Thermo Fisher/FEI holder, dimensions for a holder to fit JEOL TEMs was determined by measuring several commercial holders made for JEOL TEMs. The shaft design is considerably different than the Thermo Fisher/FEI shaft, although still relies on a dynamic o-ring which seals the shaft to the goniometer against the vacuum of the column. One difference is the tip for JEOL holders is considerably wider than the Thermo Fisher/FEI holders, allowing for a wider liquid tip which can hold more fluid lines. As a result, two additional flow lines were able to be added to the design for directed flow liquid holders for JEOL microscopes. 


\subsubsection{JEOL Shaft}

Figure 6.3a shows a JEOL holder, where critical measurement points are noted by arrows. Similar to the Thermo Fisher/FEI holder, the base of the shaft is marked by the red arrow in Figure 6.3a. The body of the holder should be flush with this surface allowing for the base to sit up against the goniometer of the microscope. Inverse of the Thermo Fisher/FEl shaft the pin on the JEOL shaft is before the sealing o-rings and is marked by the orange arrow in Figure 6.3a. This pin is responsible for triggering the airlock cycle as well as actuating the valve mechanism after the load lock cycle to allow the holder into the column. The distance from this pin to the base of the shaft is a critical dimension to ensure the holder sits at the correct location in the column. The next features are the sealing o-rings, where the JEOL shaft has 2 o-rings compared to the single on Thermo Fisher/FEI shafts. It should be noted that the size of the o-rings and groves are different sizes, although very similar. The spacing of these o-ring grooves and their distance to the base of the shaft is important to ensure proper sealing. Finally, the black arrow in Figure 6.3a shows the location of the tip which holds the sample. The distance between the imaging area to the base of the shaft should be precisely measured to ensure the sample sits in the path of the electron beam for imaging. Several commercially purchased JEOL holders were measured with electronic calipers to determine the tolerances for each dimension for replication. 
a)

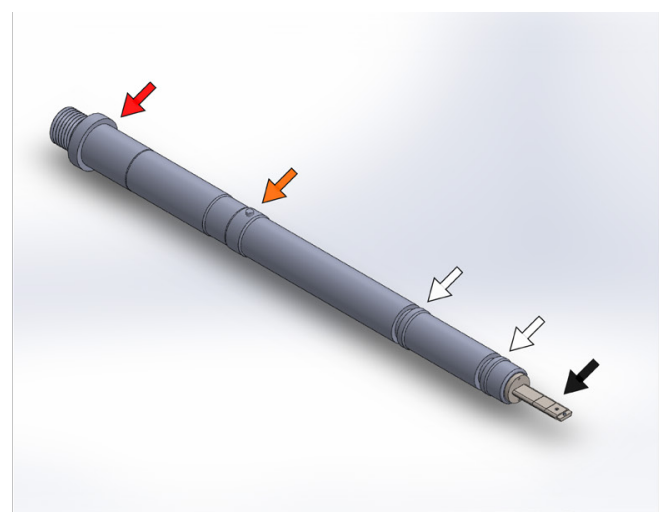

b)

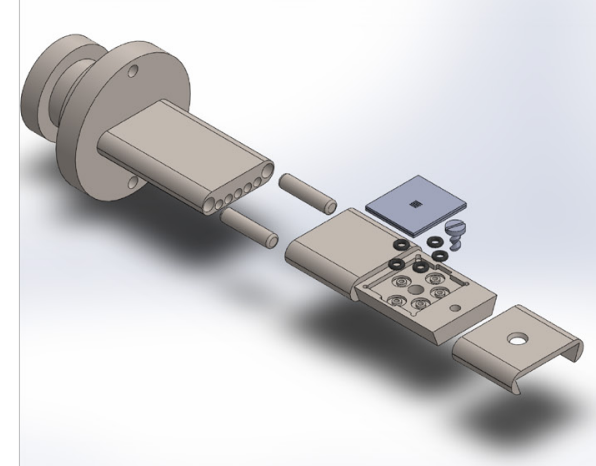

Figure 6.3: a) CAD model of a complete holder for use in a JEOL microscope. Critical dimension on the shaft are labeled by arrows, the base (red arrow), pin (orange arrow), shaft o-rings (white arrows), and imaging aperture (black arrow). b) Exploded view of 5 port holder depicting o-rings, devices, and lid. Assembly is also achieved in a two component fashion and sealed with a vacuum grade epoxy.

\subsubsection{JEOL Tip}

Figure $6.3 \mathrm{~b}$ shows an exploded version of the 5 port liquid tip to fit to the end of the JEOL shaft. In the same manner as the Thermo Fisher/FEl liquid tip it is fabricated in two sections and assembled with a vacuum grade epoxy. An o-ring groove is located on the section of the tip which interfaces with the shaft to achieve hermetic sealing, and holes on the face allow screws to secure the tip at the end of the shaft. Figure $6.3 \mathrm{~b}$ also shows how the wider tip allows for two more fluidic lines which results in larger microfabricated devices. O-rings seal against the vertical flow channels of the devices and a similar dovetail design of the tip and lid compresses the o-rings against the bottom of the device to ensure sealing. The lid is also secured by a screw which threads into a hole tapped in the end of the tip. 


\subsection{Fabrication of Nanofluidic Devices}

The devices for the directed flow holders described in section 6.2 are fabricated in a similar fashion to the devices described in chapters 4 and 5 , where free standing electron transparent membranes are fabricated by a combination of dry and wet etching. Differently however, the directed flow devices are bonded together during the fabrication process to hermetically seal the flow channel through wafer bonding techniques. As a result of the design, there are two different devices to be fabricated, an upper and a lower device. Each upper and lower device has identical masks for spacer patterning, but the mask for patterning the windows and vertical flow channels differs between the upper and lower device. The sections below describe the mask designs for both the Thermo

Fisher/FEI 3 port and JEOL 5 port directed flow holders, followed by a description of their fabrication.

\subsubsection{Design of 3 Port Devices}

Figure 6.4 shows the mask design for devices used with the Thermo Fisher/FEI 3 port holder described in section 6.2. Similar to the devices described in chapters 4 and 5 the devices are sectioned by patterning a $40 \mu \mathrm{m}$ width channel around the edges of each device. The final size of each device is $5.46 \mathrm{~mm}$ in length and $3.46 \mathrm{~mm}$ in width. Since these devices are larger than the ones described for commercial devices in chapters 4 and 5 the borders have $100 \mu \mathrm{m}$ segments left in the edges to provide stability during the etching phase to prevent the devices from falling apart too easily in the $\mathrm{KOH}$ bath. The windows are fabricated in the 
a)

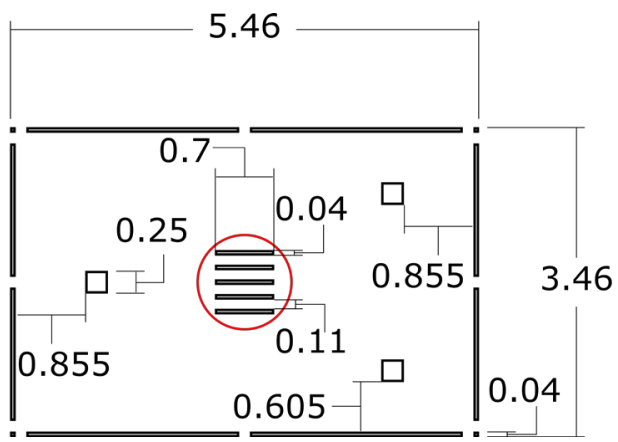

b)

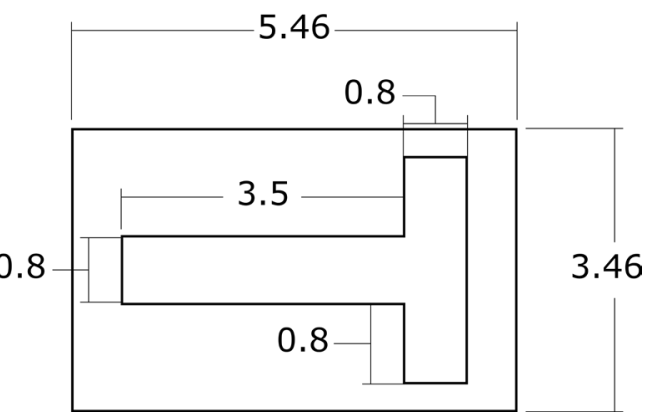

Figure 6.4: a) Mask design for multiwindow devices which for use with the 3 port holder. b) Illustration of devices produced using the mask depicted in (a).

same manner, where each window is $40 \mu \mathrm{m}$ in width, and the windows for the top device are rotated 90 degrees with respect to the bottom device. The diameter of the aperture for beam transmission is $1.25 \mathrm{~mm}$, shown by the red circle in Figure 4a. It should be noted that the size of this aperture is not constrained by a sealing o-ring due to the different sealing strategy for the directed flow holder. Therefore, it theoretically could be possible to machine a larger aperture which would allow for substantially more imaging area in future iterations. For the bottom device, vertical flow channels $250 \mu \mathrm{m}$ in width and height are patterned, which are etched simultaneously to the windows and device borders. The design in Figure $6.4 \mathrm{a}$ is of the bottom device with the vertical flow channels, where the difference in the upper device is a lack of flow channels and a 90 degree rotation of the windows.

Figure $6.4 \mathrm{~b}$ shows the mask design for patterning the spacer and bonding material, as well as dictating the flow channel. The terminal positions of the channel is the location of the vertical flow channels. Although a number of 
different designs were tested for bonding designs, the most successful design was the solid design shown in Figure 6.4b where the upper and lower devices have the same pattern design.

\subsubsection{Design of 5 Port Devices}

Figure 6.5 shows the mask design for devices which fit the JEOL 5 port directed flow holder described in section 6.2. Devices are similar to the 3 port devices described in section 6.3.1, where 5 port devices are larger to accommodate 2 extra vertical flow channels. The final device dimensions are $4.7 \mathrm{~mm}$ in width and $5.2 \mathrm{~mm}$ in length. A $40 \mu \mathrm{m}$ channel surrounds the border of the device, with 100 $\mu \mathrm{m}$ sections spaced midway down the sides and at corners to provide additional support during etching. The windows and flow channels are identical in design to the 3 port, with the addition of two extra flow channels in the 5 port. If the position of the flow channel in Figure 6.5 is not annotated it is centered with respect to the length or width axis of the device. Again, in the same way as the 3 port, the only difference in the upper device is the lack of vertical flow channels and rotation of the windows with respect to the lower device.

Figure $6.5 \mathrm{~b}$ shows the mask for patterning the flow channel and spacer/bonding material, where each channel terminates at one of the vertical flow channel locations and is also a solid film design as has been determined with optimum bonding. 


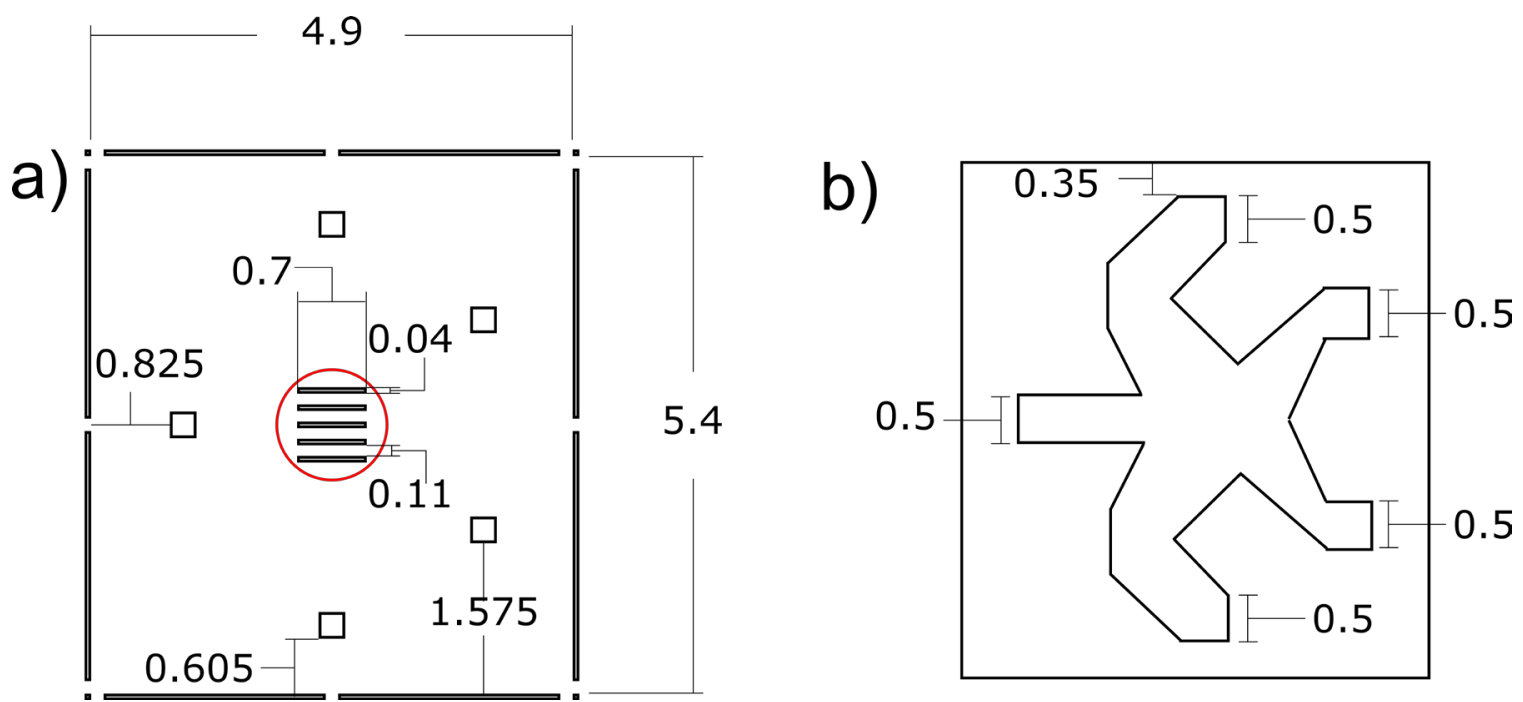

Figure 6.5: a) Mask design for multiwindow devices which for use with the 5 port holder. b) Illustration of devices produced using the mask depicted in (a).

\subsubsection{Fabrication}

Masks detailed in Figures 6.4 and 6.5 are repeated to fill a standard 4-inch wafer with a maximum number of devices. In total, 3 masks are used to pattern two wafers. The first mask patterns for thin film deposition and is used for both wafers for top and bottom devices. Another mask patterns the first wafer with windows, device borders, and vertical flow channels for the bottom devices, and the third mask patterns the second wafer with only windows and device borders.

Initially both wafers are patterned for lift off deposition of the bonding material. The masks for flow channel design in Figures $6.4 \mathrm{~b}$ and $6.5 \mathrm{~b}$ are printed as negative masks for optimal lift off. Wafers were coated with the negative photoresist AZ 5214E at $3000 \mathrm{rpm}$ for 60s. The wafers were then soft baked at $90^{\circ} \mathrm{C}$ for $2 \mathrm{~min}$, and the mask design was exposed for 2 seconds. The 
photoresist was then reverse baked for 2 minutes at $120^{\circ} \mathrm{C}$ to reverse the photoresist and the wafers were left to equilibrate at room temperature for 10 minutes. After a flood exposure for $30 \mathrm{~s}$, the wafers were developed in $A Z$ $400 \mathrm{~K}$ :water at a 1:4 ratio for about $45 \mathrm{~s}$. The wafers were then loaded into a thin film deposition unit and pumped to vacuum. One wafer was coated with a gold film, where deposition was performed at 50W DC and a deposition pressure of 5 mtorr. A 10-20nm chromium adhesion layer was deposited prior to the gold film to serve as an adhesion layer. The second wafer was coated with a thin film of silicon, where deposition was performed at 200W RF and a deposition pressure of 10 mtorr. After deposition lift off was performed in acetone with sonication followed by a soak in nanostrip $2 \mathrm{x}$ for cleaning. Thin films should be deposited with the target thickness of the final bonded devices in mind depending on application.

At this point both wafers are patterned with the same design where one wafer has a thin film of polysilicon and one wafer has a thin film of gold. One wafer is then patterned with the mask design for the lower devices containing the vertical flow channels and the other wafer is patterned with the mask design for upper devices (lacking the vertical flow channels). At this point, etching is performed identically as to the methods described in chapter 4 and 5 , where vertical flow channels, windows, and device borders are etched simultaneously. Once devices are etched and sectioned, they may be bonded together to create the hermetic seal and complete the flow channel. 


\subsubsection{Wafer Bonding}

Bonding of wafers to achieve hermetic sealing is a challenging process that is highly dependent on surface roughness and sensitive to cleanliness (236). Direct bonding strategies in particular require very low surface roughness, often below 1 $\mathrm{nm} \mathrm{rms}$, and minimal wafer bow to achieve wafer level bonding $(155,158,159)$. Chemical-mechanical polishing can be used to planarize surfaces to below this critical surface roughness, although the clean room this work was performed in lacked this capability. While magnetron deposition can generally produce thin films with very good surface roughness, a minimum surface roughness of $2 \mathrm{~nm}$ rms for deposited silicon oxide was achieved which was not able to be bonded using direct bonding techniques even when using plasma activation strategies described in literature. Thermocompression systems are also an option, where high heat and pressure are used to bond two surfaces together by softening the thin films and forcing them into contact (153). The high pressures used are often able to overcome limitations in surface roughness. Gold thermocompression was attempted for wafer bonding, although it was found that while the wafer bonder used for this work could achieve the temperatures needed for thermocompression bonding it could not reach high enough pressures to achieve adequate bonding.

Eutectic systems may also be used for wafer bonding, which are advantageous since bonding can be achieved at lower temperatures (167). Gold and silicon each have melting temperatures greater than $1000^{\circ} \mathrm{C}$, but have a eutectic 
temperature around $370^{\circ} \mathrm{C}(165,237,238)$. This allows for bonding at temperatures and pressures that are achievable with the instrumentation available for this work. For fabrication of directed flow devices, the thin films patterned on the wafers were gold and silicon. Wafer level bonding is advantageous as the bonding is performed before etching of the free standing windows and sectioning of the devices. This helps reduce potential surface contaminants that can affect the bonding efficiency as well as improve the chances the fragile windows survive the bonding process. A downside however to etching the windows after bonding is that the $\mathrm{KOH}$ etch will also etch the thin film of silicon in the bonding layer. Multiple attempts were made to bond wafers together but at each point during the $\mathrm{KOH}$ etch step for window formation enough $\mathrm{KOH}$ was able to etch laterally along the sputtered silicon thin film that the wafers became unbonded during the etch process.

As a result, window etching and device sectioning was performed prior to bonding, and individual devices were bonded together. This significantly reduced throughput and increases the chances of windows breaking during the bonding phase but was able to avoid the limitations of the silicon thin film etching away during the etch step. Individual devices were cleaned in nanostrip $2 x$ at $110^{\circ} \mathrm{C}$ and then assembled in a jig to ensure alignment of the devices. The temperature was increased to $450^{\circ} \mathrm{C}$ which was higher than the eutectic point of the system to increase fluidity of the bonding films, and the platen pressure was increased to $4000 \mathrm{~N}$. Bonding was tested at different time intervals, where the best results 
were found for devices which were bonded for 60 minutes. After 60 minutes at $450^{\circ} \mathrm{C}$ the temperature was turned off, although the pressure was not released. Cooling of the platens generally takes several hours, where the platen pressure was not released until the temperature had dropped below $200^{\circ} \mathrm{C}$ to reduce mechanical and thermal stress on the bonded films.

Figure 6.6 shows 3 port devices which were bonded using the Au-Si eutectic system with the strategy described above. Figures $6.6 a-b$ are devices that were bonded and separated after bonding. While regions of the gold and silicon thin films can be seen to have melted and bonded together there are regions that remained unbonded. As a result, hermeticity was not achieved with this device when it was pumped down to vacuum and did not seal properly. Figures $6.6 \mathrm{c}-\mathrm{d}$ are devices that were bonded at the same conditions, but when tested did pump down to appropriate vacuum values for TEM imaging, and liquid was found over the windows. After the experiment, the devices were broken apart to inspect the surface of the films. As is seen in the images, enough force was applied to break the silicon substrate before the bond between the thin films was broken. Over the rest of the devices the bonding is seen to have much better coverage and uniformity across the films.

While bonding can be achieved, another failure mode for the process described here is the membranes being damaged during the bonding phase. Figure $6.6 e-f$ show images of windows after bonding, where the window in $6.6 \mathrm{e}$ can be seen to have a tear in it while the window in $6.6 \mathrm{f}$ is intact despite identical bonding 

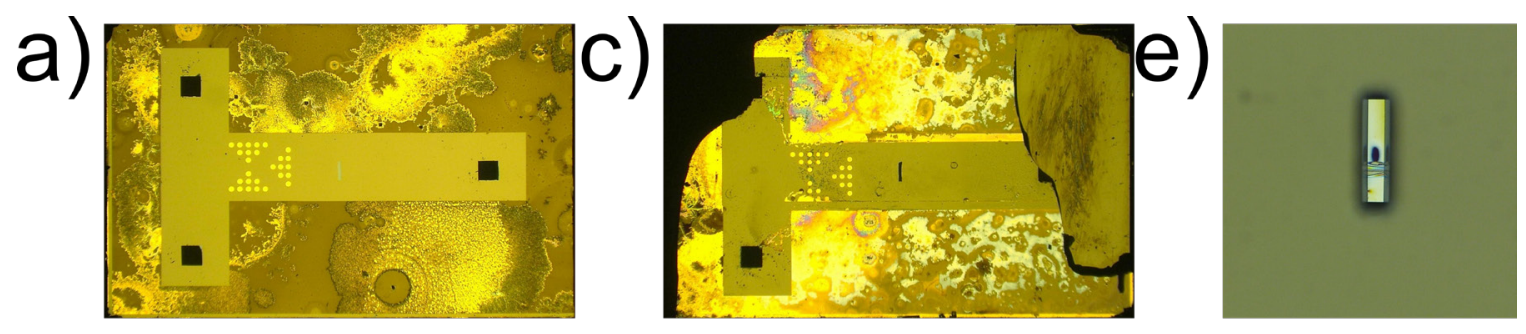

b)
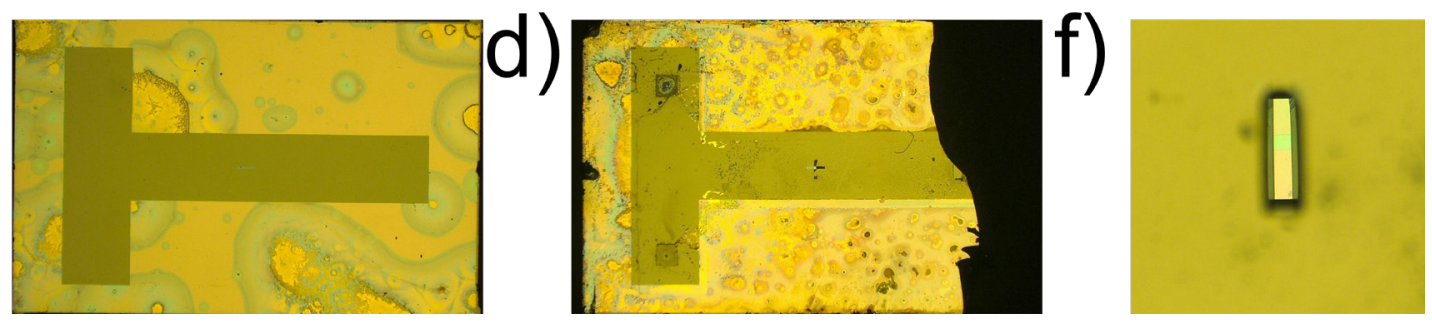

Figure 6.6: a-b) Upper and lower devices separated after bonding where sealing was not achieved. Incomplete bonding regions are visible in the silicon and gold films. c-d) Upper and lower devices separated after bonding where sealing was achieved. In some areas the silicon substrate broke before the bond did. e) Image of a broken window after bonding. f) Image of intact windows after bonding.

processes. The breaking of windows and incomplete bonding of some samples currently remains a challenge of the described process. Despite these limitations, bonded devices which were usable for LC-TEM experiments were achieved.

\subsection{Demonstration of Nanoparticle Mixing}

To demonstrate the ability for two initially separate samples to be introduced to the imaging area through each inlet channel and make their way to the imaging area, a nanoparticle flow experiment was performed. One liquid line flowed 50 $\mathrm{nm}$ silver nanoparticles, while the second liquid line flowed $100 \mathrm{~nm}$ gold nanoparticles. The holder and devices were loaded into the microscope and imaged using HAADF-STEM imaging. The nanoparticle solutions were pumped into the holder using a syringe pump at a rate of $0.5 \mu \mathrm{L} / \mathrm{min}$. Figure $6.7 \mathrm{a}-\mathrm{f}$ show frames from a video acquired during particle flow, where particles are seen over the window area from the very first frame. Each frame shown in Figure 6.7 is 25 
a)

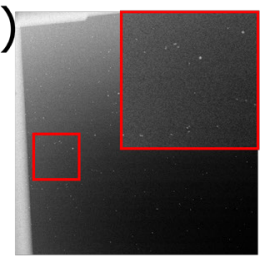

d)

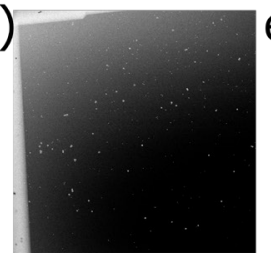

b)

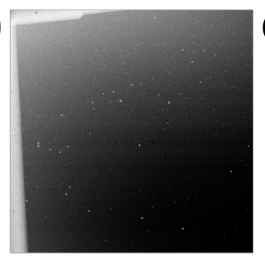

e)

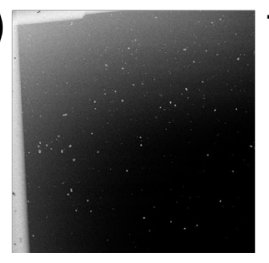

c)

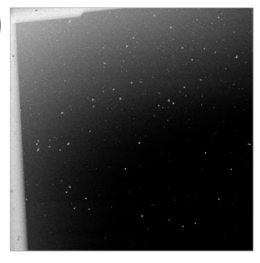

f)

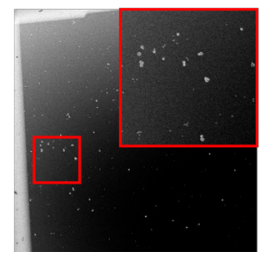

g)

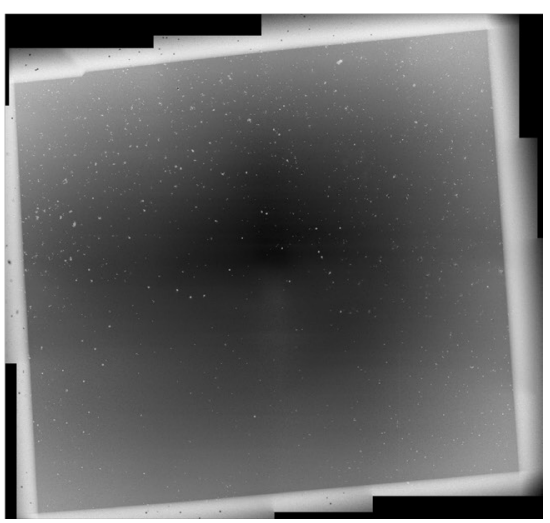

Figure 6.7: a-f) Frames of a window in a directed flow holder during the flow of 100nm gold and 200 silver nanoparticles from different inlet lines. Both particle sizes are observed over the window area over the course of the experiment indicating mixing of the two solutions. g) Low magnification image of the entire window area after the experiment depicting many nanoparticles successfully flowed to the imaging area.

STEM scans apart, where the time of 1 scan is approximately 3 seconds. The motion of individual particles was not captured due to the slow temporal resolution of the scans, but particles were clearly seen coming into view in locations where particles were not previously. After capturing the flow video, images were acquired sequentially over the entire window area, and they were stitched together to form the overview shown in Figure 6.7g. This demonstrates the ability not only for two unique samples to be directly flowed into a sealed nanofluidic device, but also that the distance between their inlet ports and the imaging area is far enough for the sample to diffusively mix into each other such that both samples are imaged together at the image plane.

Some improvements and optimizations may still be possible with the bonding processes described here to increase throughput success rate. Additional 
instrumentation may allow for better bonding strategies to be performed at the wafer level which would drastically improve the throughput of the process. Structures to bond the windows together to reduce window bulging effects may also be advantageous and could be performed during the wafer bonding step. These research areas will be the focus of my post-doctoral studies, where optimizing fabrication and bonding conditions will allow for enhanced science to be performed on biological samples with LC-TEM. Ultimately, these devices offer enhanced control of thickness reproducibility and environmental control for flowing and mixing samples in a manner not possible with other current commercially available systems. 


\section{Chapter 7: Optimizing Imaging of Low Contrast, Beam Sensitive Samples in LC-TEM}

\subsection{Introduction}

In Chapter 5 the increased sensitivity of biological samples to the electron beam in LC-TEM was demonstrated, where beam driven morphological changes were observed with electron fluxes as low as $1 \mathrm{e}^{-} / \AA^{2}$. While irradiation thresholds for the functional inactivation of proteins, enzymes, DNA, and other structural components of a cell remain unknown, it is likely that they will require substantially lower electron fluxes than those used for cryo-EM (239). As such, any imaging performed at irradiation levels below those that damage cells will likely have very poor contrast and poor SNR due to low signal levels. Identifying imaging modalities that provide the strongest contrast forming mechanisms and highest SNR values is critical to making the most out of the limited signal that will be used for image formation. In this chapter a comparison of different imaging modalities, SNR, and damage, is described for both cryo-EM and LC-TEM to compare the new methods against more established approaches.

\subsection{Comparison of Cryogenic and Liquid Imaging}

The majority of published biological structures determined with cryo-EM and tomography have been performed with BF-TEM, where the use of phase plates (45) and energy filters can further improve image contrast. Especially for imaging larger structures such as whole cells, the large depth of focus of BF-TEM allows for structures through the thickness of the sample to remain in focus. A 
disadvantage of using BF-TEM for very thick samples however is the increasing effect of chromatic aberration on resolution with sample thickness $(54,219)$. With increasing sample thickness, the number of electrons which have experienced an inelastic collision increases. These inelastically scattered electrons are focused to different focal points by the objective lens compared with the elastically scattered electrons resulting in image aberrations which decreases the resolution of the sample. With multiple energy loss collisions, the coherence of the inelastically scattered electrons decreases such that they no longer contribute to the contrast of the image through constructive or deconstructive interference with the other electrons in the image. This chromatic aberration has been shown to have a significant impact on the resolution of images collected from thick sections of resin embedded cells (54) and has been simulated for liquids (219).

STEM imaging can alternatively be used for imaging, where effects of chromatic aberration are not seen due to the lack of a post specimen objective lens. While TEM imaging is generally practically limited to bright field imaging, STEM imaging can collect signal for high angle annular dark field (HAADF), annular dark field (ADF), and bright field (BF) imaging, which may often be collected simultaneously. HAADF-STEM is often used for atomic resolution imaging of materials samples, as the $z$ contrast image it produces can be advantageous over high resolution BF-TEM of similar samples. While STEM imaging has been performed on biological samples for negative stained resin embedded samples (54), and cryo-EM (52). While STEM imaging does not suffer from chromatic 
aberration effects seen in BF-TEM, beam broadening effects can decrease resolution for very thick samples (240). As the resolution of a STEM image depends on the size of the probe, the broadening of the STEM probe through a very thick sample will decrease the resolution of structures towards the bottom of the sample. Furthermore, classic STEM uses very high convergence angles to minimize probe size for atomic resolution imaging. While this reduces the size of the probe, it results in a very narrow depth of focus, which for very thick samples introduces defocus artifacts in the final image (241).

Low convergence angle STEM imaging is an alternative strategy for imaging thick, low contrast samples to overcome issues of defocus artifacts. This has been discussed extensively in theory $(51,219,240-242)$, and demonstrated experimentally to give advantageous contrast and resolution over BF-TEM for resin embedded and cryo-EM sections of cells $(52,54)$. While the application of low STEM imaging for LC-TEM has been discussed (119), a comparison of imaging modalities for cells imaged with LC-TEM has not yet been performed experimentally. Furthermore, for the published work on whole cells imaged with LC-TEM images obtained lack resolution and detail when compared with whole cell imaging done on cryo-EM samples. It is unclear why this discrepancy exists between LC-TEM and cryo-EM images, whether simply the effect of large thicknesses in LC-TEM or if there are other contributing factors which degrade image resolution and contrast. 
To address this limitation the organism Cupriavidus metallidurans was imaged with both LC-TEM and cryo-EM and across several imaging modalities to determine which modalities give the strongest contrast and signal to noise ratio (SNR). Cells were imaged with bright field TEM (BF-TEM), energy filtered TEM (EFTEM), low convergence angle STEM and high convergence angle STEM. To accurately quantify differences between images it is important to ensure that thicknesses between samples remains consistent. Chapter 5 demonstrated the effect of changing thicknesses on image contrast and SNR, where internal cellular structure is lost with increasing sample thickness. To control for changes in thickness electron energy loss spectroscopy was used to quantify the sample thickness for every image obtained. The thickness can be estimated in inelastic mean free paths (IMFP), which is measured using the log ratio method $(124,243$, 244). For reference, $300 \mathrm{keV}$ electrons have an IMFP of $\sim 180 \mathrm{~nm}$ in pure water.

Additionally, for each imaging modality the incident electron flux was kept constant at $1 \mathrm{e}^{-/} \AA^{2}$ to ensure that comparison between different imaging modalities observes differences in image formation differences rather than differences in electron flux. While the field of view differs slightly in each image due to differences in magnification and image projection for different detectors, the total electron flux for each image was kept constant. For TEM imaging, both BF-TEM and EFTEM, the condenser aperture was $150 \mu \mathrm{m}$, the objective aperture was $40 \mu \mathrm{m}$, the beam current was $3.3 \mathrm{nA}$, the beam diameter was 11.45 um, and the exposure time was $1 \mathrm{~s}$. For STEM imaging, the condenser aperture 
a)

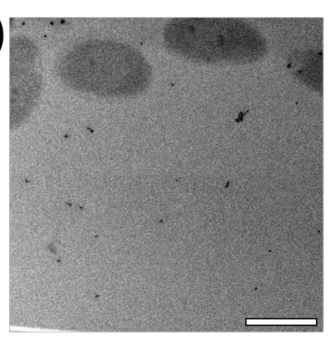

e)

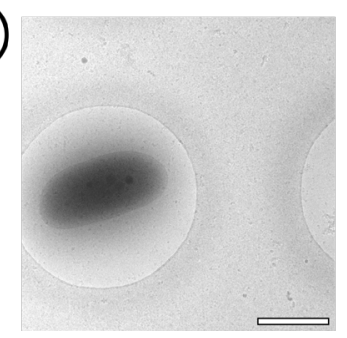

b)

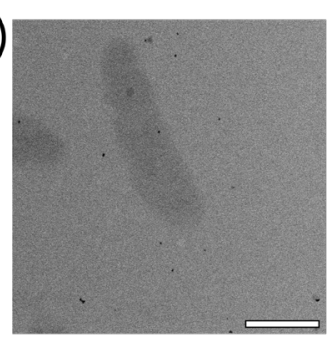

f)

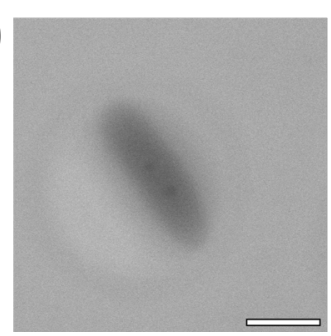

c)

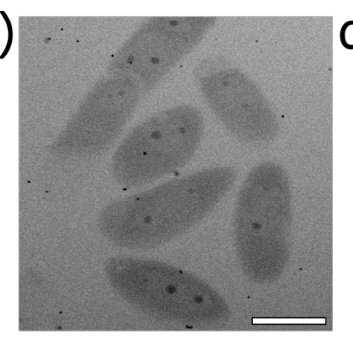

d)

g)

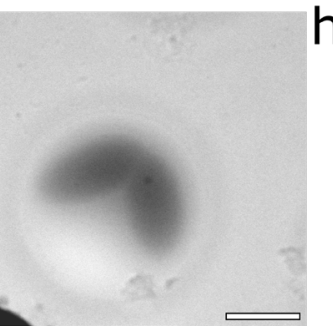

h)

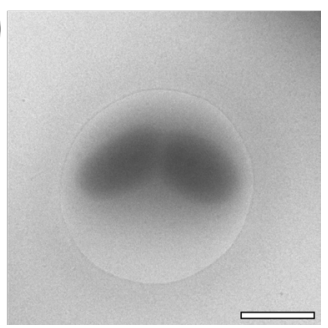

Figure 7.1: a) BF-TEM in liquid. b) Low convergence angle BF-STEM in liquid. c) High convergence angle BF-STEM in liquid. d) EFTEM in liquid. e) BF-TEM in ice. f) Low convergence angle BF-STEM in ice. g) High convergence angle BF-STEM in ice. h) EFTEM in ice. Scale bars are all $1 \mu \mathrm{m}$.

was $50 \mu \mathrm{m}$, the beam current was $21 \mathrm{pA}$, the pixel dwell time was $3.2 \mu \mathrm{s}$, and the semi-convergence angle was $17.8 \mathrm{mrad}$ or $5 \mathrm{mrad}$.

\subsubsection{BF-TEM}

Figure 7.1a shows a BF-TEM image of cells acquired in a liquid cell. The measured thickness of the water layer near the cells was 1.96 IMFP. Contrast in the image is strong, and the cells are clearly visible against the background water in the sample. Some low contrast regions are visible in the cell, and particles of different contrast are associated with some of the cells. Figure 7.1e shows a comparable BF-TEM image of a cell obtained with cryo-EM. The measured thickness of the ice near the cell was 1.58 IMFP, making it the thinnest of all the images shown in Figure 7.1. The cell membranes are clearly visible, and the cell has strong contrast against the background. 


\subsubsection{High Convergence Angle STEM}

Typical STEM imaging on the instrument used is performed with a convergence semi-angle of $17.8 \mathrm{mrad}$. This allows for a very small probe to be formed for atomic resolution imaging, but results in considerable geometric resolution degradation for samples which are out of focus (219). The narrow depth of focus for high convergence angle probes results in only a thin slice of a thick samples being in true focus while the rest of the sample will have out of focus imaging artifacts. Figure 7.1b shows a BF-STEM image taken with high convergence angle STEM imaging, where the thickness of the sample near the cell was 2.93 IMFP. This was the thickest of all the samples imaged and displayed the poorest SNR and contrast. The cell is only weakly visible against the background although some high dentistry structures are still visible associated with the cell. Figure $7.1 \mathrm{f}$ shows a cryo-EM image of a cell imaged with high convergence angle BF-STEM where the thickness of the ice near the cell was 2.03 IMFP. Similar to the LC-TEM image the cell is only weakly visible against the background of ice and carbon, although some high density structures are still associated with the cell.

\subsubsection{Low Convergence Angle STEM}

By changing the strength of the condenser lens and decreasing the size of the condenser aperture just before the sample plane (typically condenser lens 2 ) the semi-convergence angle of the electron probe can be reduced. With a lower convergence angle the depth of focus can be increased, where defocus 


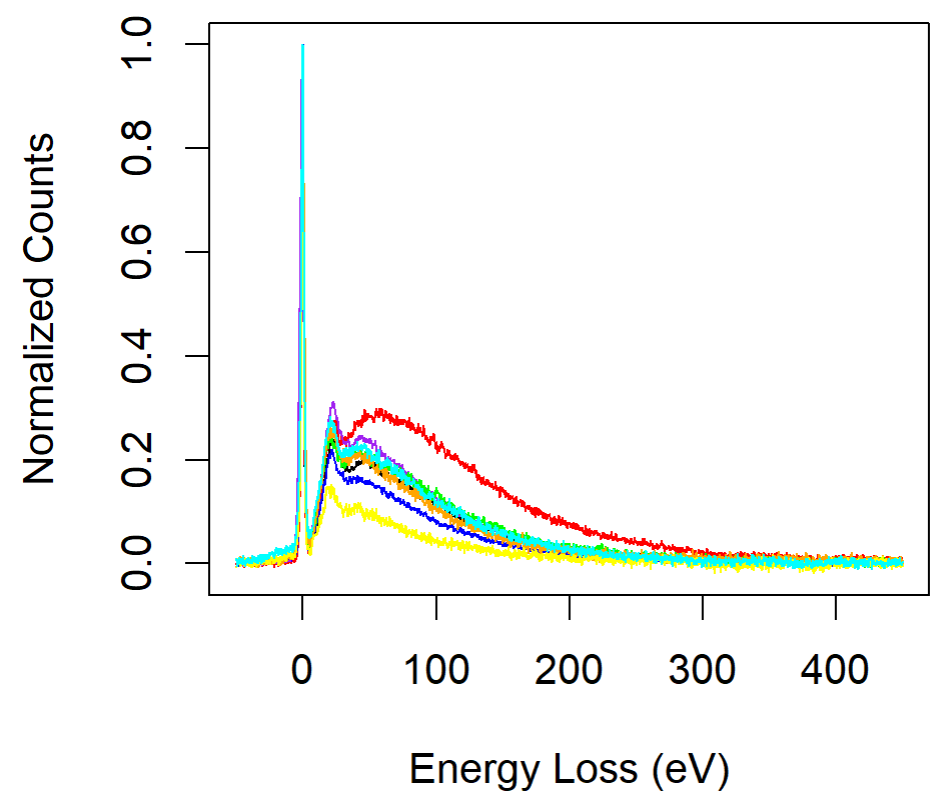

Figure 7.2: Electron energy loss spectra from locations near cells in Figure 7.1, where the thickness of each sample was similar.

aberrations contribute less to resolution degradation in the final image.

Furthermore, the use of low convergence angles may result in stronger contrast in the BF image, converse to typical STEM imaging where HAADF-STEM often gives the image with best SNR. Figure 7.1c shows a BF-STEM, with a $5 \mathrm{mrad}$ semi-convergence angle, image of cells imaged in a liquid cell where the thickness of liquid near the cells was 2.29 IMFP. Cells have good contrast against the liquid background and both structures of low density and high density are observed associated with the cells. Figure $7.1 \mathrm{~g}$ shows an image of cells imaged with low convergence angle BF-STEM frozen in vitrified ice where the 
thickness near the cells was 2.27 IMFP. Contrast of the cells is similarly strong against the ice background and high density structures are visible associated with the cells.

\subsubsection{Energy Filtered TEM}

The use of an electromagnetic prism can be used to separate electrons by their energies after passing through a sample, where electrons which have undergone inelastic collisions and experienced an energy loss will be focused to a different point than their elastically scattered neighbors. The collection of this spectra of electrons is the basis of electron energy loss spectroscopy (EELS) where the energy loss during an inelastic collision is directly related to the energy of the electron orbital that the collision originated from allowing for elemental analysis of a sample. In addition to spectral information, the electrons can also be filtered by use of a "slit" which only allows electrons from a specified portion of the spectrum through. These electrons can then be used to form an image using only the electrons with energies specified by the position and size of the slit and is known as energy filtered TEM (EFTEM). While in theory the energy slit can be placed on the carbon edge to maximize contrast from the cell (composed primarily of carbon), the reduction in signal with this technique requires large integration times which can considerably increase the incident electron exposure of the sample. As LC-TEM imaging is likely to be limited by the electron irradiation sensitivity of biological samples, imaging strategies which require large incident electron fluxes are not practical for image formation strategies. Another option is 
to place the slit on the zero-loss peak, which is the portion of electrons which have not experienced an inelastic collision and have not lost any energy. Importantly, the elastically scattered electrons from a sample are incoherent and do not contribute to the constructive/deconstructive interference of scattered electrons for phase contrast imaging, and as such only contribute to the noise signal in the image. By forming the image with only the elastically scattered electrons the SNR of the image can be improved by removing the inelastically scattered electrons.

Figure 7.1d shows an EFTEM image of cells in a liquid cell where the thickness near the cell was 2.14 IMFP. The width of the slit was $30 \mathrm{eV}$ and was centered on the zero-loss peak for image formation. Contrast for the cells is strong against the liquid background and both low density and high density structures are visible associated with the cells. Figure $7.1 \mathrm{~h}$ shows cells frozen in a layer of vitreous ice and imaged with the same parameters as LC-TEM images where the ice thickness near the cells was 1.92 IMFP. Cells have strong contrast against the ice background similar to LC-TEM images and high density structures are visible associated with the cells.

Figure 7.2 shows the EELS spectrums for each image from which the thickness in mean free paths was calculated from. In general, the thickness of all samples was around 2-2.3 IMFP. It is important to note that these thicknesses are not ideal as a result of sample thickness limitations of LC-TEM samples. As a result, the cryo-EM sample ice thicknesses were intentionally made thicker than ideal in 
order for accurate comparison to the LC-TEM data. Automated plunge freezing of cryo-EM samples has allowed for highly reliable and thin ice thicknesses to be formed, where ideal thicknesses for cryo-EM of cells of $C$. metallidurans would be closer to 1-1.3 IMFP. The increase in thickness for both ice and liquid causes considerable loss of structural information, and as a result is not indicative of what may be maximally achieved with LC-TEM, but rather a comparison of imaging modalities at equivalent thicknesses. As LC-TEM grows as a technique and better instrumentation and sample preparation methods are implemented it may eventually be possible to achieve liquid samples which are closer to ideal thicknesses for maximizing resolution.

\subsection{Quantification of Signal to Noise Ratio}

While Figure 7.1 provides a qualitative comparison of differences between electron imaging modalities on image contrast and SNR, the visual representation of the image can be altered by the brightness and contrast settings of the image which is chosen by the individual presenting the data. In order to avoid potentially subjective presentation, and interpretation of the images the SNR was quantified for each image in order to directly compare differences between electron imaging modalities. In order to quantify the signal to noise of a single image both the noise component and signal component of the image must be known. Unfortunately, the type, distribution, and magnitude of noise in an EM image is unknown, and so the contribution of intensity from signal or from noise cannot be directly measured. The SNR can however be estimated 
a)
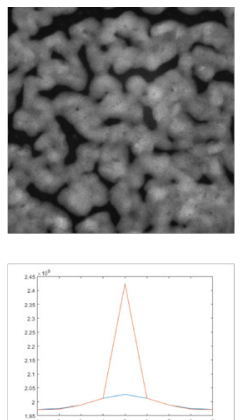
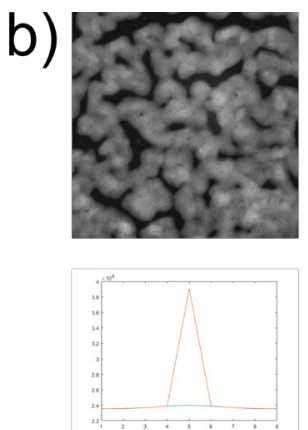

C)

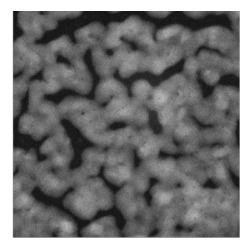

d)

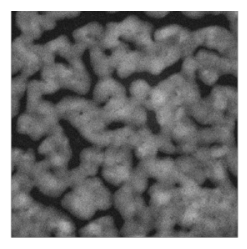

e)
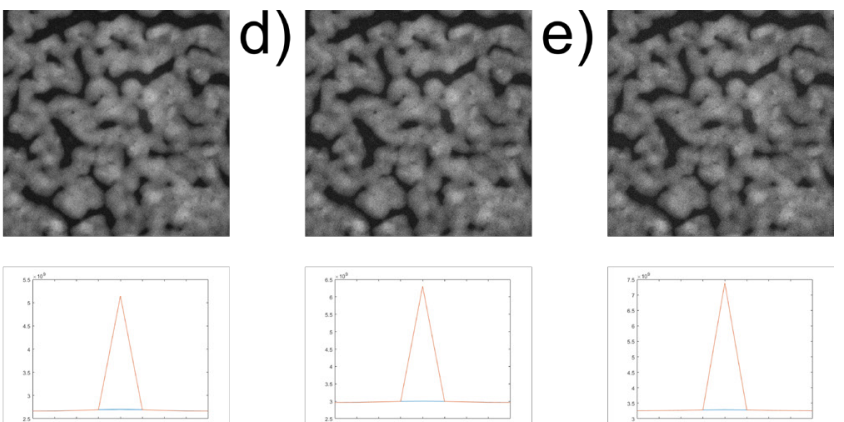

Figure 7.3: a-e) HAADF-STEM images with added white gaussian noise with increasing variance of 0.1 , along with the two dimensional sample of the 2D ACF and fitted gaussian curve for each image.

using the 2-dimensional autocorrelation function (ACF) of the image to estimate the signal power of the image, as has been described previously (245).

$$
S N R=\frac{\Phi_{11}{ }^{N F}(0,0)-\mu_{1}{ }^{2}}{\Phi_{11}(0,0)-\Phi_{11}{ }^{N F}(0,0)}
$$

Equation 7.1 shows the calculation for SNR estimation from a single image where the noise energy $\left(\Phi_{11} \mathrm{NF}(0,0)\right)$ and signal energy $\left(\Phi_{11}(0,0)\right)$ can be determined from the 2-dimensional ACF of the image (245). The mean value of the image $\left(\mu 1^{2}\right)$ is measured simply as the mean pixel intensity of the entire image. Estimation was done using MATLAB, where 8bit images were read in as 2D arrays of pixel intensity values from $0-255$. The two dimensional autocorrelation function for the image was computed a gaussian curve was plotted around the maxima of the ACF (at the origin), where the noise energy of the image is the value of the ACF at the origin and the signal energy is the ACF value at the gaussian curve fit to the data. 
Table 7.1: Estimated SNR Values from Images with Added Noise

\begin{tabular}{lccccc} 
Figure & $\mathrm{a}$ & $\mathrm{b}$ & $\mathrm{c}$ & $\mathrm{d}$ & $\mathrm{e}$ \\
\hline White Noise Variance & 0 & 0.01 & 0.02 & 0.03 & 0.04 \\
SNR & 4.8863 & 1.5743 & 1.0975 & 0.9046 & 0.7990 \\
\hline
\end{tabular}

With the noise energy, signal energy, and image mean determined the SNR of an individual image can be estimated. To confirm that the implementation of the SNR estimation method is working as described a single STEM image was duplicated and gaussian noise was added to the image with a known variance.

Figure 7.3 shows these images, where the noise variance increased by 0.01 for each image in a-e. The simplified sections of the 2D ACF around the maximum value (at the origin) is shown for each image, where the orange line is the ACF values and the blue line is a gaussian curve fit to the data to estimate the signal power of the image. The calculated SNR is shown in Table 7.1, where it can be seen that the SNR decreases with increasing noise variance. This indicates that the described strategy for estimating the SNR of a single image was working as implemented.

Table 7.2 shows the estimated SNR values for each image in Figure 7.1 using the single image SNR estimation method described above, where Figure 7.4 shows SNR estimation process for the BR-TEM liquid and cryo-EM images. Of all the electron imaging modalities tested it was found that BF-TEM and EFTEM had the highest estimated SNR compared with STEM imaging. EFTEM likely 
a)

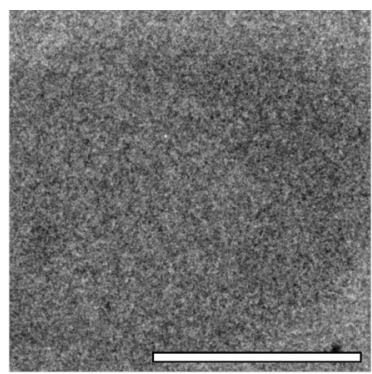

d)

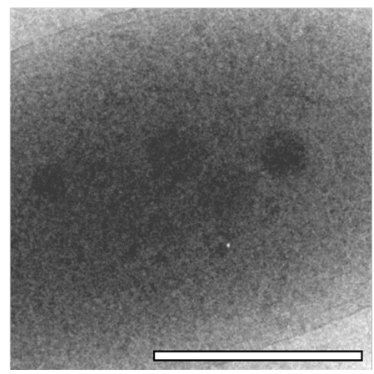

b)

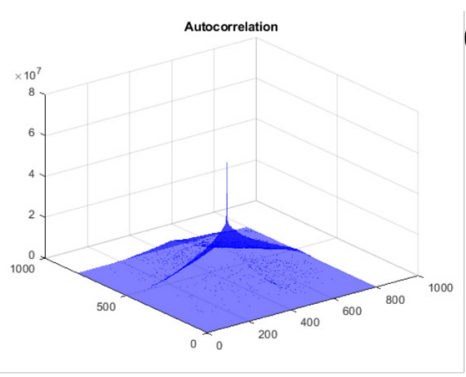

e)

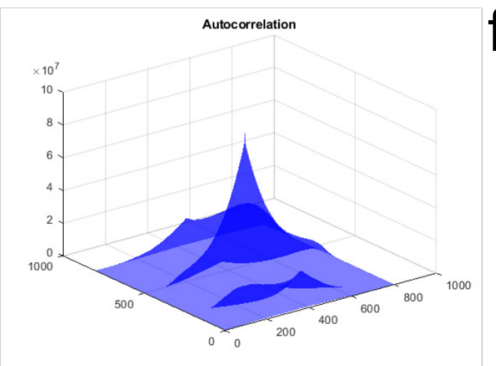

c)

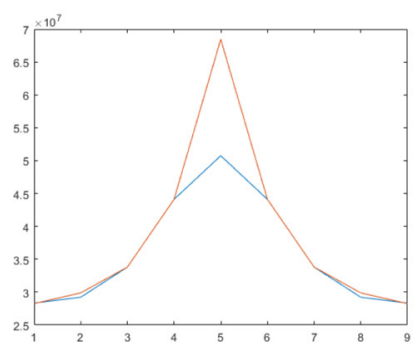

f)

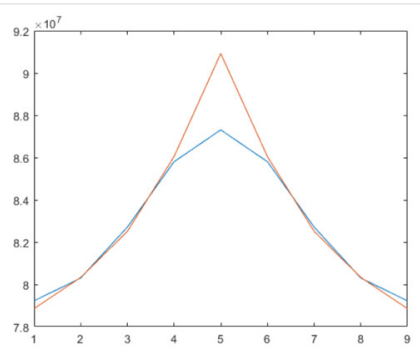

Figure 7.4: a) Cropped view corresponding to Figure 7.la used to estimate SNR. b) 2D ACF plot of (a). c) ACF values sampled along the $\mathrm{x}$-axis of the ACF maximum (orange line) and the fitted gaussian curve for signal energy estimation. d-f) Same as (a-c) but for the image in Figure 7.1e. Scale bars are $500 \mathrm{~nm}$.

provides stronger SNR due to the filtering of inelastically scattered electrons by only forming the image from the zero loss peak. These inelastically scattered electrons do not contribute to phase contrast through constructive and deconstructive interference as a result of their incoherence with the elastically scattered electrons in the image and as such only contribute to noise. BF-TEM likely has slightly better SNR due to the increased signal as all of the electrons are collected for the image. 
Table 7.2: Estimated SNR Values from LC-TEM and Cryo-EM Images

\begin{tabular}{ccccccc} 
& & BF-STEM & $\begin{array}{c}\text { HAADF- } \\
\text { STEM }\end{array}$ & $\begin{array}{c}\text { BF-STEM } \\
\alpha=5 \mathrm{mrad}\end{array}$ & $\begin{array}{c}\text { HAADF- } \\
\alpha=5 \mathrm{mrad}\end{array}$ & EFTEM \\
\hline BC-TEM & 1.8116 & 0.4892 & 0.4533 & 0.5683 & 0.5229 & 12.0492 \\
& & & & & & \\
Cryo-EM & 16.7694 & 1.7283 & 0.8921 & 3.9663 & 1.1996 & 15.9064 \\
\hline
\end{tabular}

$\alpha=$ semi-convergence angle of the STEM probe

Although BF-TEM and EFTEM provide the strongest SNR, there may be instances where STEM imaging is preferred over TEM. In particular, theoretical simulations have suggested that the resolution loss to beam broadening in STEM is not as severe as the resolution loss to chromatic aberration (219). The resolution was not quantified for the images in Figure 7.1 and as such it may be that for thicker samples STEM imaging will provide higher resolution than TEM imaging. Table 7.1 shows that for both cryo-EM and LC-TEM images that low convergence angle STEM provides better SNR when compared with high convergence angle STEM images. HAADF-STEM images can be collected simultaneously with BF-STEM images, and for each BF-STEM image in Figure 7.1 corresponding HAADF-STEM images were collected (not shown). The SNR was estimated for these images as well and for each the SNR was stronger in the BF image. This trend for low convergence angle BF-STEM providing the strongest contrast and SNR is in agreement with theoretical $(51,219,241)$ and experimental demonstrations on cryo-EM and resin embedded thin sections (52, 54). As such, if maximal SNR is desired than it appears BF-TEM or EFTEM 
should be used, while if resolution is desired than low convergence angle BFSTEM should be used.

One intriguing observation was that for all images in Figure 7.1, the cryo-EM images consistently had the highest estimated SNR compared with their liquid counterparts despite achieving similar thicknesses for each sample. For the images collected, the cryo-EM images were always collected with the cell over a hole in the holey carbon film, while the LC-TEM images were always collected with two $10 \mathrm{~nm} \mathrm{SiN}$ membranes. It may be that the additional scattering from the membranes, although thin, is enough to account for the decrease in SNR in these images. STEM image simulation of nanoparticles imaged with and without the presence of silicon nitride membranes has demonstrated an increase in image SNR which increases as a function of membrane thickness (246). Further advances in materials used to form the free standing membranes to decrease their contribution to electron scattering in combination with decreasing their thick may eventually allow for LC-TEM images to have similar SNR as cryo-EM. There may also be additional explanations for the difference in SNR between cryo-EM and LC-TEM images, where the additional vibrational states of the water molecules in LC-TEM compared with the ice in cryo-EM may play some role in generating additional noise in the images. The physical scattering differences between liquid water and ice however is not well understood, and will require further characterization to be certain of some contribution from the differences in phase of water. 
Another observation made of the differences between LC-TEM images and cryoEM images was that cells in LC-TEM were commonly observed to have large, low density structures associated with the cells. Both cells in cryo-EM and LCTEM had high density structures of varying size and contrast (likely gold nanoparticles and energy storage bacterial microcompartments) but only the LCTEM images showed cells with low density structures. It may be that these structures are the result of the cells reacting to being trapped within the hermetically sealed chamber which has limited gas exchange and no exchange of fresh nutrient media to the imaging area. Some bacteria are known to form gas vacuoles which allow them to float closer to the air-water interface when sensing anaerobic growth conditions. It may be the structures seen here are the result of nutrient deprivation within the confines of the liquid cell, which has implications for how the morphology of cells can be driven or altered by the geometry of the device limiting liquid and gas exchange. Further characterization of this phenomena will be critical to observing artifact free dynamics of living cells with LC-TEM.

\subsection{Electron Irradiation Comparison Between Cryogenic and Liquid Samples}

As discussed in chapter 5 , the role of electron irradiation damage of cells is another critical component affecting accurate interpretation of LC-TEM images. For the damage series shown in chapter 5 , the cell is shown to shrink with electron irradiation exposures as low as $1 \mathrm{e}^{-/} / \AA^{2}$. Notably, this electron flux is 
considerably lower than fluxes used for cryo-EM imaging and tomography of whole cells which can achieve nanometer level spatial resolution $(40,63)$. These cells were grown in a nutrient broth media which was supplemented with $50 \mu \mathrm{M}$ AuCl. C. metallidurans is remarkably known for its ability to biomineralize environmental gold to form valence zero metallic nanoparticles (206-208). While the exact mechanism of this biomineralization pathway is not well understood, where the inclusion of the $\mathrm{AuCl}$ in these samples was intended to shed light on how these organisms perform the biomineralization process. It was considered however that the addition of heavy metal clusters to the growth media could cause additional damage in the sample by causing a local increase in secondary damage during electron irradiation. Cryo-EM studies have demonstrated a local increase in radiation damage observed around metal binding regions of proteins (247) and LC-TEM experiments have shown increased damage at the interface of higher z-number materials that support the hypothesis that the presence of 50 $\mu \mathrm{M} \mathrm{AuCl}$ could accelerate irradiation damage of the cells imaged. Alternatively, C. metallidurans has also been shown to upregulate genes involved in oxidative stress response when exposed to environmental $\mathrm{Au}(208)$. It is possible that the upregulation of this pathway may enable the cell to scavenge the radical species produced through radiolysis, which may in turn reduce the effect of beam driven damage of the cell. To test if damage is appreciably accelerated or mitigated by the presence of $\mathrm{AuCl}$ in the growth media the damage experiments from chapter 5 were repeated on cells which were grown without $\mathrm{AuCl}$ supplemented in the growth media. Additionally, to compare damage mechanisms between cryo-EM 
and LC-TEM these damage experiments were repeated for samples which were frozen in vitrified ice and imaged with identical beam conditions as the LC-TEM samples. Cells were imaged with a single frame flux of $1 \mathrm{e}^{-} / \AA^{2}$, where the beam was blanked for 20 minutes, and the cell was imaged again at the same flux. This was repeated for 4 images and a total cumulative electron flux of $4 \mathrm{e}^{-1} / \AA^{2}$ by the final frame. Figure 7.5 shows the results of these imaging series where $7.5 \mathrm{a}$ and 7.5b are LC-TEM images and $7.5 \mathrm{c}$ and $7.5 \mathrm{~d}$ are cryo-EM images. Figures $7.5 \mathrm{a}$ and $7.5 \mathrm{c}$ are cells which have been supplemented with $50 \mu \mathrm{M} \mathrm{AuCl}$ in the growth media while Figures $7.5 \mathrm{~b}$ and $7.5 \mathrm{~d}$ are grown without supplemented gold. For both LC-TEM images cells are observed to begin shrinking in size after the first 1 $\mathrm{e}^{-} / \AA^{2}$, illustrated by overlaying the first and last images in the final column in Figure 7.5. While it may be that the presence of gold in the growth media may still impact the rate of damage, it is not significant enough to be visually apparent as shown qualitatively in the images shown. When compared with the images acquired with cryo-EM, cells do not exhibit any obvious morphological changes after a cumulative electron flux of $4 \mathrm{e}^{-/} / \AA^{2}$ when frozen in a layer of vitreous ice. Importantly, the lack of observable damage for cryo-EM images does not indicate the absence of radiation damage, only that the damage has not propagated to manifest as observable changes within the resolution achieved in the image. Radiolysis is certainly still taking place in these images, but the radical species and broken bonds produced by electron irradiation are locked in place by the frozen ice layer in cryo-EM $(198,233)$, while in LC-TEM they are free to 


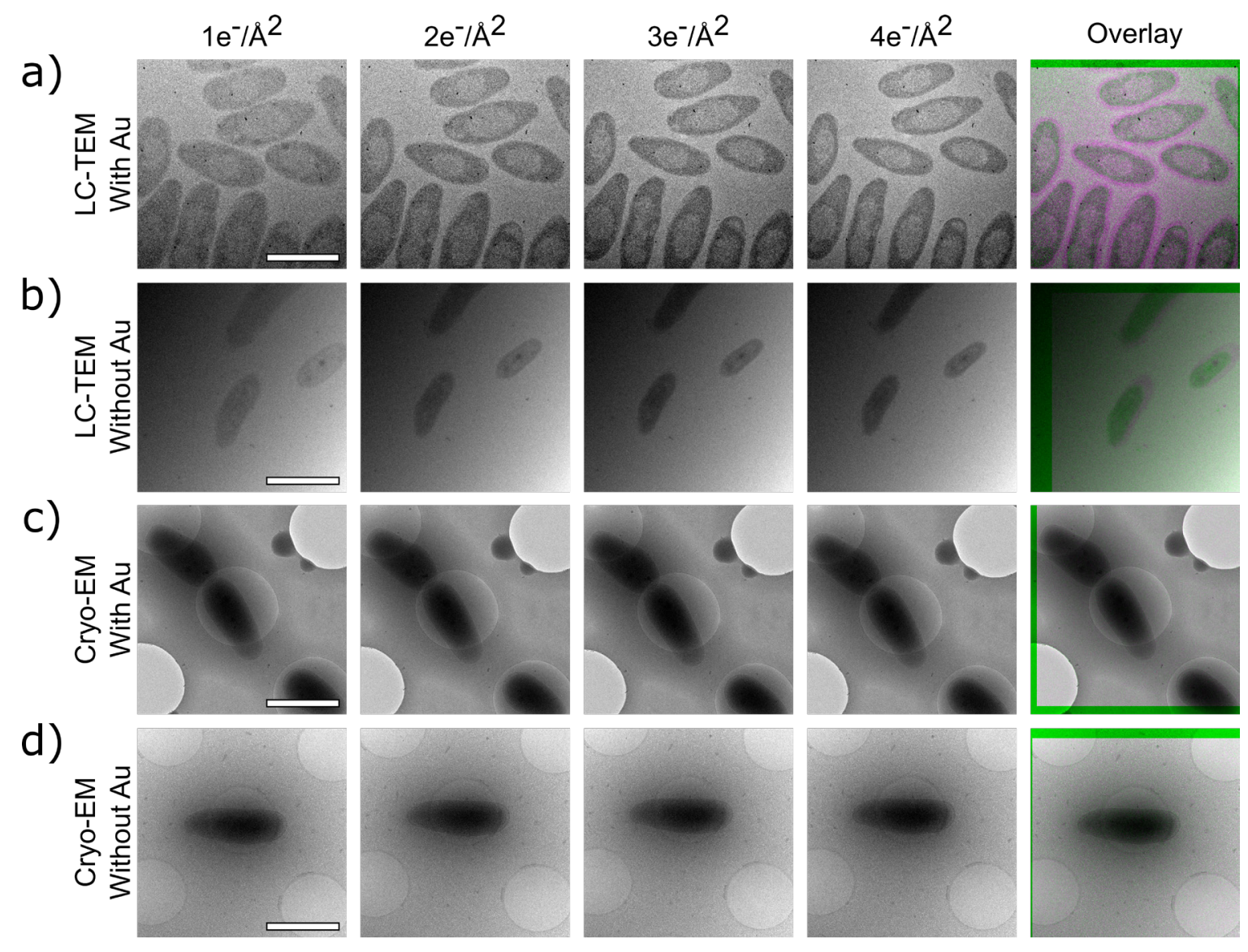

Figure 7.5: a) Damage series of C. metallidurans in LC-TEM grown in media supplemented with $50 \mu \mathrm{M}$ gold chloride. b) Same as (a) but for cells grown without gold chloride. c-d) Same as (a-b) but in cryo. Scale bars are $1000 \mathrm{~nm}$.

propagate and react with other components of the cell manifesting in visible changes to cell ultrastructure.

To further demonstrate the differences between irradiation damage manifestation between LC-TEM and cryo-EM, cells frozen in vitrified ice were imaged at much higher electron fluxes until the effects of damage were clearly visible in the images acquired. Figure 7.6 shows selected frames from a cryo-EM damage series of C. metallidurans in $10 \mathrm{e}^{-/} \AA^{2}$ increments for cells grown supplemented with $\mathrm{AuCl}$ (Figure 7.6a) and without $\mathrm{AuCl}$ (Figure 7.6b). The onset of damage is 


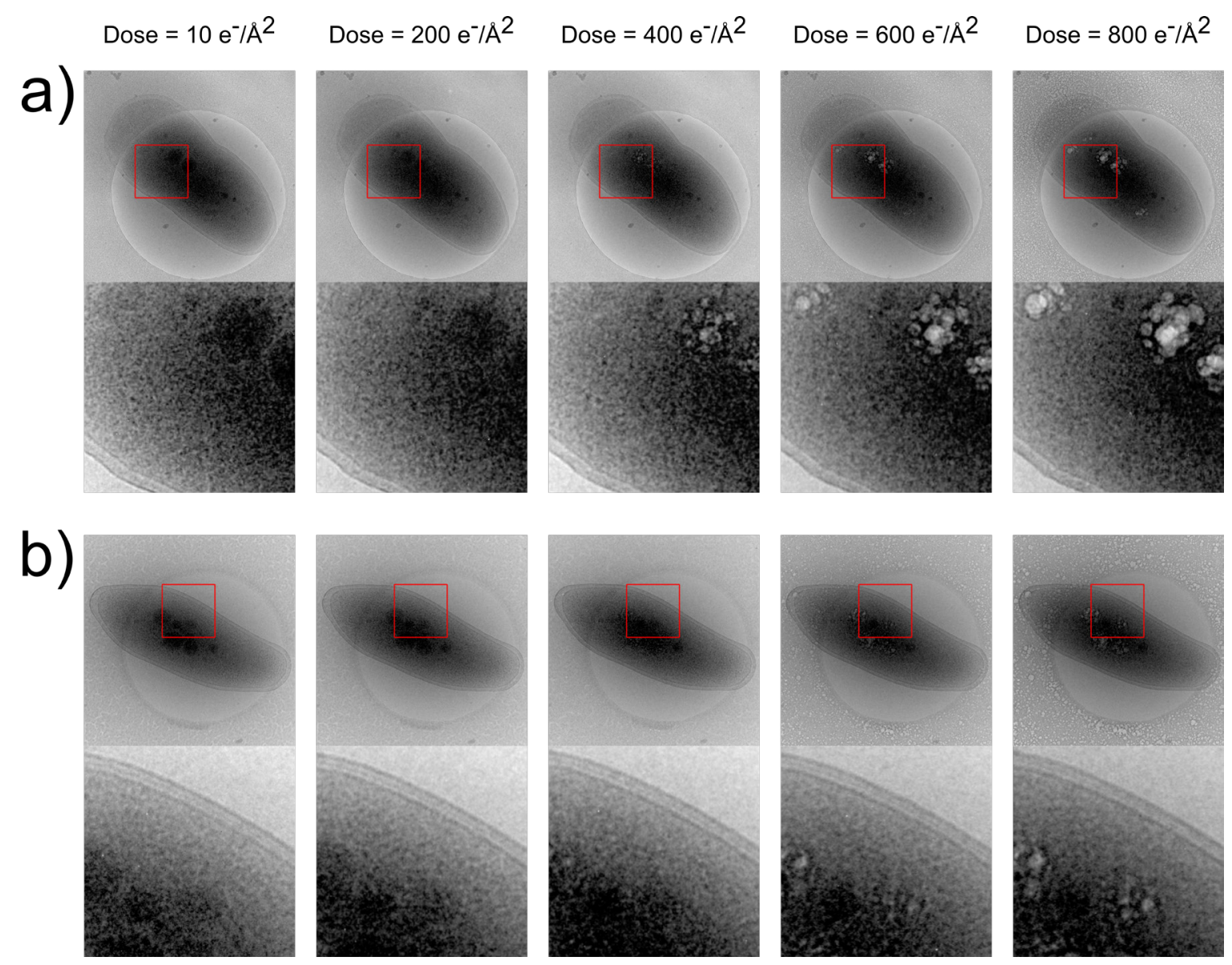

Figure 7.6: a) Cryo-EM damage series of $C$. metallidurans grown in media supplemented with $50 \mu \mathrm{M}$ gold chloride. b) Cryo-EM damage series of $C$. metallidurans grown without gold chloride.

apparent around $300-400 \mathrm{e}^{-} / \AA^{2}$, where damage manifestation is apparent as bubble formation. This differs considerably both in onset (from $1 \mathrm{e}^{-/} \AA^{2}$ in LC-TEM to $\sim 300 \mathrm{e}^{-/} / \AA^{2}$ in cryo-EM) as well as manifestation where LC-TEM damage appears as cell shrinking and cryo-EM damage appears as bubble formation. Both bubble formation in cryo-EM and cell shrinking in LC-TEM has been described in literature $(116,193)$, supporting the observation of distinct differences between damage manifestation between these two techniques. 
These results have important implications for performing LC-TEM imaging on biological structures, as it demonstrates that the well characterized relationship between electron irradiation and structural damage is not necessarily directly translatable to LC-TEM experiments. Critically, while the loss of structure resolution with increasing electron flux has been documented in detail for cryoEM (248), the relationship between loss of structure and a corresponding loss of function of a protein, enzyme, DNA, or other biomolecule is not known. While dehydrated catalase proteins have been demonstrated to lose functionality with as low as $0.05 \mathrm{e}^{-/} / \AA^{2}$, the presence of water and corresponding radical species produced during electron irradiation in LC-TEM may impact the irradiation sensitivity of cells and biomolecules during liquid cell experiments. Perhaps most importantly, the dose conditions for imaging whole cells with cryo-EM are not directly applicable to LC-TEM experiments. Further characterization of the critical electron flux threshold which results in inactivation of enzymatic function is the next step for determining dose tolerance of organisms and other biological samples to ensure that observations in LC-TEM experiments are representative of phycological behavior and not the result of beam driven phenomena. 


\section{Chapter 8: Conclusions and Future Outlook}

The work detailed in the earlier chapters of this dissertation outline my efforts at developing techniques and instrumentation for holistic structural characterization of organisms and macromolecular structures in cryogenic and liquid samples. At the smallest scale, cell-free expression of soluble and membrane proteins was demonstrated to serve as a viable platform for biomass production and structural studies. At the largest scale, whole cell cryo-electron tomography was performed to pave a route towards in vivo imaging of molecular structures within a whole cell context. Future cryo-EM work will be focused on acquiring single particle data on DGAT and high quality tomograms of $O$. tauri and $C$. metallidurans using direct electron detectors and phase plates for high quality reconstructions and to empower visual proteomics and work towards creating a true continuum for ultrastructural analysis from single molecules to whole cell context.

At the outset of this dissertation, work with LC-TEM was originally envisioned as a tool for characterizing physiological processes of biomineralization. Throughout the course of the work it became apparent that the role of the electron beam and its interaction with liquid and biological samples was poorly understood. As a result, the LC-TEM work described in this dissertation reflects my attempts to characterize the interactions and chemistry changes that occur in liquids with biological and chemical samples under electron irradiation. For biological samples, while electron damage of cryogenic samples has been well described in literature, it is clear from the damage experiments performed in Chapters 5 
and 7 that the damage mechanisms and thresholds cannot be assumed to be directly transferable from the cryo-EM field. As such, the next steps for realizing biological imaging with LC-TEM is to characterize the inactivation thresholds of enzymes, proteins, DNA, and other biomolecules to identify the imaging conditions which need to be used in order to avoid introducing damage artifacts that can confound interpretation and analysis of images.

Finally, continued technology and instrumentation improvement will be a necessary component of future work for both the cryo-EM and LC-TEM fields. Optimization of the directed LC-TEM flow holder described in Chapter 6 will permit expanded reproducibility and generation of meaningful statistics to facilitate physiological interpretation. Improvements in electron cameras and detectors, along with novel imaging regimes to maximize contrast in weakly scattering materials will also be critical in realization of LC-TEM for biological material.

During the above work, I expanded both my scientific repertoire as well as my technical skill set. I developed expertise in biology, biochemistry, cell biology, chemistry, computing, engineering, fluidics, genetics, microfabrication, molecular biology, optical microscopy and transmission electron microscopy. I plan to continue pushing the science frontiers listed above as part of my post-doctoral work to continue to expand and improve bioimaging capabilities for both cryogenic and liquid samples using electron microscopy in hopes of one day achieving my goal of understanding biosystems across scales. 


\section{Reference List}

1. R. E. Franklin, R. G. Gosling, Molecular configuration in sodium thymonucleate. Nature 171, 740-741 (1953).

2. J. D. Watson, F. H. Crick, Molecular structure of nucleic acids; a structure for deoxyribose nucleic acid. Nature 171, 737-738 (1953).

3. U. Resch-Genger, M. Grabolle, S. Cavaliere-Jaricot, R. Nitschke, T. Nann, Quantum dots versus organic dyes as fluorescent labels. Nat Methods 5, 763-775 (2008).

4. T. Schmidt, G. J. Schutz, W. Baumgartner, H. J. Gruber, H. Schindler, Imaging of single molecule diffusion. Proceedings of the National Academy of Sciences 93, 2926-2929 (1996).

5. G. Seisenberger et al., Real-time single-molecule imaging of the infection pathway of an adeno-associated virus. Science 294, 1929-1932 (2001).

6. B. N. Giepmans, S. R. Adams, M. H. Ellisman, R. Y. Tsien, The fluorescent toolbox for assessing protein location and function. Science 312, 217-224 (2006).

7. G. W. Li, X. S. Xie, Central dogma at the single-molecule level in living cells. Nature 475, 308-315 (2011).

8. H. Murakoshi et al., Single-molecule imaging analysis of Ras activation in living cells. Proc Natl Acad Sci US A 101, 7317-7322 (2004).

9. C. W. Freudiger et al., Label-free biomedical imaging with high sensitivity by stimulated Raman scattering microscopy. Science 322, 1857-1861 (2008).

10. B. G. Saar et al., Label-free, real-time monitoring of biomass processing with stimulated Raman scattering microscopy. Angew Chem Int Ed Engl 49, 54765479 (2010).

11. B. O. Leung, K. C. Chou, Review of super-resolution fluorescence microscopy for biology. Appl Spectrosc 65, 967-980 (2011).

12. B. Huang, M. Bates, X. Zhuang, Super-resolution fluorescence microscopy. Annu Rev Biochem 78, 993-1016 (2009).

13. E. Betzig et al., Imaging intracellular fluorescent proteins at nanometer resolution. Science 313, 1642-1645 (2006).

14. D. Greenfield et al., Self-organization of the Escherichia coli chemotaxis network imaged with super-resolution light microscopy. PLoS Biol 7, e1000137 (2009). 
15. M. J. Rust, M. Bates, X. Zhuang, Sub-diffraction-limit imaging by stochastic optical reconstruction microscopy (STORM). Nat Methods 3, 793-795 (2006).

16. B. Huang, W. Wang, M. Bates, X. Zhuang, Three-dimensional super-resolution imaging by stochastic optical reconstruction microscopy. Science 319, 810-813 (2008).

17. C. Jacobsen, Soft x-ray microscopy. Trends in Cell Biology 9, $44-47$ (1999).

18. W. Chao, B. D. Harteneck, J. A. Liddle, E. H. Anderson, D. T. Attwood, Soft Xray microscopy at a spatial resolution better than $15 \mathrm{~nm}$. Nature 435, 1210-1213 (2005).

19. D. Shapiro et al., Biological imaging by soft x-ray diffraction microscopy. Proc Natl Acad Sci U S A 102, 15343-15346 (2005).

20. M. A. Le Gros, G. McDermott, C. A. Larabell, X-ray tomography of whole cells. Curr Opin Struct Biol 15, 593-600 (2005).

21. J. L. Carrascosa et al., Cryo-X-ray tomography of vaccinia virus membranes and inner compartments. $J$ Struct Biol 168, 234-239 (2009).

22. C. A. Larabell, M. A. Le Gros, X-ray tomography generates 3-D reconstructions of the yeast, saccharomyces cerevisiae, at 60-nm resolution. Mol Biol Cell 15, 957-962 (2004).

23. J. R. Helliwell, Synchrotron X-radiation protein crystallography: instrumentation, methods and applications. Reports on Progress in Physics 47, 1403-1497 (1984).

24. B. S. Schuwirth et al., Structures of the bacterial ribosome at 3.5 A resolution. Science 310, 827-834 (2005).

25. O. Kennard et al., The Crystal and Molecular Structure of Adenosine Triphosphate. Proceedings of the Royal Society A: Mathematical, Physical and Engineering Sciences 325, 401-436 (1971).

26. R. F. Tilton, J. C. Dewan, G. A. Petsko, Effects of temperature on protein structure and dynamics: $\mathrm{x}$-ray crystallographic studies of the protein ribonucleaseA at nine different temperatures from 98 to 320K. Biochemistry 31, 2469-2481 (2002).

27. M. Frank et al., Femtosecond X-ray diffraction from two-dimensional protein crystals. IUCrJ 1, 95-100 (2014).

28. T. Kimura et al., Imaging live cell in micro-liquid enclosure by X-ray laser diffraction. Nat Commun 5, 3052 (2014). 
29. A. Barty, J. Kupper, H. N. Chapman, Molecular imaging using X-ray freeelectron lasers. Annu Rev Phys Chem 64, 415-435 (2013).

30. S. G. Boxer, M. L. Kraft, P. K. Weber, Advances in imaging secondary ion mass spectrometry for biological samples. Annu Rev Biophys 38, 53-74 (2009).

31. A. M. Herrmann et al., Nano-scale secondary ion mass spectrometry - A new analytical tool in biogeochemistry and soil ecology: A review article. Soil Biology and Biochemistry 39, 1835-1850 (2007).

32. K. J. Carpenter, A. Horak, L. Chow, P. J. Keeling, Symbiosis, morphology, and phylogeny of Hoplonymphidae (Parabasalia) of the wood-feeding roach Cryptocercus punctulatus. J Eukaryot Microbiol 58, 426-436 (2011).

33. P. Hansma, V. Elings, O. Marti, C. Bracker, Scanning tunneling microscopy and atomic force microscopy: application to biology and technology. Science 242, 209-216 (1988).

34. R. Henderson, The potential and limitations of neutrons, electrons and X-rays for atomic resolution microscopy of unstained biological molecules. $Q$ Rev Biophys 28, 171-193 (1995).

35. K. Wuthrich, Protein structure determination in solution by NMR spectroscopy. $J$ Biol Chem 265, 22059-22062 (1990).

36. M. Winey, J. B. Meehl, E. T. O'Toole, T. H. Giddings, Jr., Conventional transmission electron microscopy. Mol Biol Cell 25, 319-323 (2014).

37. L. F. Kourkoutis, J. M. Plitzko, W. Baumeister, Electron Microscopy of Biological Materials at the Nanometer Scale. Annual Review of Materials Research 42, 33-58 (2012).

38. K. R. Porter, A. Claude, E. F. Fullam, A Study of Tissue Culture Cells by Electron Microscopy : Methods and Preliminary Observations. J Exp Med 81, 233-246 (1945).

39. S. Watanabe et al., Ultrafast endocytosis at mouse hippocampal synapses. Nature 504, 242-247 (2013).

40. A. Muller et al., Ultrastructure and complex polar architecture of the human pathogen Campylobacter jejuni. Microbiologyopen 3, 702-710 (2014).

41. D. B. Williams, C. B. Carter, Transmission Electron Microscopy. (Springer, 2009). 
42. M. Knoll, E. Ruska, Das Elektronenmikroskop. Zeitschrift fr Physik 78, 318-339 (1932).

43. M. Knoll, E. Ruska, Beitrag zur geometrischen Elektronenoptik. Annalen der Physik, 607 (1932).

44. K. Nagayama, R. Danev, Phase-plate electron microscopy: a novel imaging tool to reveal close-to-life nano-structures. Biophys Rev 1, 37-42 (2009).

45. W. Dai et al., Zernike phase-contrast electron cryotomography applied to marine cyanobacteria infected with cyanophages. Nat Protoc 9, 2630-2642 (2014).

46. R. N. Clough, G. Moldovan, A. I. Kirkland, Direct Detectors for Electron Microscopy. Journal of Physics: Conference Series 522, (2014).

47. S. J. Pennycook et al., Scanning Microscopy for Nanotechnology. Scanning Transmission Electron Microscopy for Nanostructure Characterization (Springer).

48. N. D. Browning et al., Scanning transmission electron microscopy an experimental tool for atomic scale interface science. Reviews on Advanced Materials Science 1, 1-26 (2000).

49. E. M. James, N. D. Browning, Practical aspects of atomic resolution imaging and analysis in STEM. Ultramicroscopy 78, 125-139 (1999).

50. O. N. Antzutkin, R. D. Leapman, J. J. Balbach, R. Tycko, Supramolecular Structural Constraints on Alzheimer's $\beta$-Amyloid Fibrils from Electron Microscopy and Solid-State Nuclear Magnetic Resonance $\dagger$. Biochemistry 41, 15436-15450 (2002).

51. P. Rez, T. Larsen, M. Elbaum, Exploring the theoretical basis and limitations of cryo-STEM tomography for thick biological specimens. J Struct Biol 196, 466478 (2016).

52. S. G. Wolf et al., 3D visualization of mitochondrial solid-phase calcium stores in whole cells. Elife 6, (2017).

53. A. A. Sousa, R. D. Leapman, Development and application of STEM for the biological sciences. Ultramicroscopy 123, 38-49 (2012).

54. M. F. Hohmann-Marriott et al., Nanoscale 3D cellular imaging by axial scanning transmission electron tomography. Nat Methods 6, 729-731 (2009).

55. R. F. Egerton, Electron energy-loss spectroscopy in the TEM. Reports on Progress in Physics 72, (2009). 
56. M. A. Aronova, R. D. Leapman, Development of Electron Energy Loss Spectroscopy in the Biological Sciences. MRS Bull 37, 53-62 (2012).

57. J. S. Wu et al., Imaging and elemental mapping of biological specimens with a dual-EDS dedicated scanning transmission electron microscope. Ultramicroscopy 128, 24-31 (2013).

58. T. Malis, S. C. Cheng, R. F. Egerton, EELS log-ratio technique for specimenthickness measurement in the TEM. J Electron Microsc Tech 8, 193-200 (1988).

59. A. Al-Amoudi et al., Cryo-electron microscopy of vitreous sections. EMBO J 23, 3583-3588 (2004).

60. J. L. Milne et al., Cryo-electron microscopy--a primer for the non-microscopist. FEBS J 280, 28-45 (2013).

61. I. Hurbain, M. Sachse, The future is cold: cryo-preparation methods for transmission electron microscopy of cells. Biol Cell 103, 405-420 (2011).

62. J. Dubochet et al., Cryo-electron microscopy of vitrified specimens. $Q R e v$ Biophys 21, 129-228 (1988).

63. Y. W. Chang et al., Architecture of the type IVa pilus machine. Science 351, aad2001 (2016).

64. A. Bartesaghi et al., 2.2 A resolution cryo-EM structure of beta-galactosidase in complex with a cell-permeant inhibitor. Science 348, 1147-1151 (2015).

65. D. Sirohi et al., The 3.8 A resolution cryo-EM structure of Zika virus. Science 352, 467-470 (2016).

66. M. McElwee, S. Vijayakrishnan, F. Rixon, D. Bhella, Structure of the herpes simplex virus portal-vertex. PLoS Biol 16, e2006191 (2018).

67. Y. W. Chang et al., Architecture of the Vibrio cholerae toxin-coregulated pilus machine revealed by electron cryotomography. Nat Microbiol 2, 16269 (2017).

68. D. F. Parsons, Structure of wet specimens in electron microscopy. Improved environmental chambers make it possible to examine wet specimens easily. Science 186, 407-414 (1974).

69. E. Ruska, Beitrag zur übermikroskopischen Abbildung bei höheren Drucken. Kolloid-Zeitschrift 100, 212-219 (1942).

70. I. M. Abrams, J. W. McBain, A Closed Cell for Electron Microscopy. Journal of Applied Physics 15, 607-609 (1944). 
71. M. J. Williamson, R. M. Tromp, P. M. Vereecken, R. Hull, F. M. Ross, Dynamic microscopy of nanoscale cluster growth at the solid-liquid interface. Nat Mater 2, 532-536 (2003).

72. C. Cassidy et al., Water without windows: Evaluating the performance of open cell transmission electron microscopy under saturated water vapor conditions, and assessing its potential for microscopy of hydrated biological specimens. PLoS One 12, e0186899 (2017).

73. T. W. Huang et al., Self-aligned wet-cell for hydrated microbiology observation in TEM. Lab Chip 12, 340-347 (2012).

74. D. B. Peckys, G. M. Veith, D. C. Joy, N. de Jonge, Nanoscale imaging of whole cells using a liquid enclosure and a scanning transmission electron microscope. PLoS One 4, e8214 (2009).

75. E. A. Ring, D. B. Peckys, M. J. Dukes, J. P. Baudoin, N. de Jonge, Silicon nitride windows for electron microscopy of whole cells. J Microsc 243, 273-283 (2011).

76. A. J. Leenheer, J. P. Sullivan, M. J. Shaw, C. T. Harris, A Sealed Liquid Cell for In Situ Transmission Electron Microscopy of Controlled Electrochemical Processes. Journal of Microelectromechanical Systems 24, 1061-1068 (2015).

77. E. A. Ring, N. de Jonge, Microfluidic system for transmission electron microscopy. Microsc Microanal 16, 622-629 (2010).

78. M. J. Dukes et al., Improved microchip design and application for in situ transmission electron microscopy of macromolecules. Microsc Microanal 20, 338-345 (2014).

79. C. Wadell et al., Nanocuvette: A Functional Ultrathin Liquid Container for Transmission Electron Microscopy. ACS Nano 11, 1264-1272 (2017).

80. M. Tanase et al., High-Resolution Imaging and Spectroscopy at High Pressure: A Novel Liquid Cell for the Transmission Electron Microscope. Microsc Microanal 21, 1629-1638 (2015).

81. E. Jensen, A. Burrows, K. Molhave, Monolithic chip system with a microfluidic channel for in situ electron microscopy of liquids. Microsc Microanal 20, 445451 (2014).

82. J. M. Yuk et al., High-resolution EM of colloidal nanocrystal growth using graphene liquid cells. Science 336, 61-64 (2012). 
83. E. Khestanova, F. Guinea, L. Fumagalli, A. K. Geim, I. V. Grigorieva, Universal shape and pressure inside bubbles appearing in van der Waals heterostructures. Nat Commun 7, 12587 (2016).

84. Q. Chen et al., 3D motion of DNA-Au nanoconjugates in graphene liquid cell electron microscopy. Nano Lett 13, 4556-4561 (2013).

85. K. H. Nagamanasa, H. Wang, S. Granick, Liquid-Cell Electron Microscopy of Adsorbed Polymers. Adv Mater 29, (2017).

86. H. Cho et al., The Use of Graphene and Its Derivatives for Liquid-Phase Transmission Electron Microscopy of Radiation-Sensitive Specimens. Nano Lett 17, 414-420 (2017).

87. I. N. Dahmke et al., Graphene Liquid Enclosure for Single-Molecule Analysis of Membrane Proteins in Whole Cells Using Electron Microscopy. ACS Nano 11, 11108-11117 (2017).

88. Y. Inayoshi, H. Minoda, A carbon sandwich environmental cell for wet specimens. Microscopy (Oxf) 62, 623-628 (2013).

89. J. Park et al., Direct Observation of Wet Biological Samples by Graphene Liquid Cell Transmission Electron Microscopy. Nano Lett 15, 4737-4744 (2015).

90. C. Wang, Q. Qiao, T. Shokuhfar, R. F. Klie, High-resolution electron microscopy and spectroscopy of ferritin in biocompatible graphene liquid cells and graphene sandwiches. Adv Mater 26, 3410-3414 (2014).

91. H. Wang, K. H. Nagamanasa, Y. J. Kim, O. H. Kwon, S. Granick, Longer-Lasting Electron-Based Microscopy of Single Molecules in Aqueous Medium. ACS Nano, (2018).

92. H. Jang et al., Nanofluidic chip for liquid TEM cell fabricated by parylene and silicon nitride direct bonding. Nanotechnology 28, 375301 (2017).

93. J. M. Grogan, H. H. Bau, The Nanoaquarium: A Platform for In Situ Transmission Electron Microscopy in Liquid Media. Journal of Microelectromechanical Systems 19, 885-894 (2010).

94. C. Mueller, M. Harb, J. R. Dwyer, R. J. D. Miller, Nanofluidic cells with controlled pathlength and liquid flow for rapid high resolution in situ imaging with electrons. The Journal of Physical Chemistry Letters 4, (2013).

95. J. F. Creemer et al., A MEMS Reactor for Atomic-Scale Microscopy of Nanomaterials Under Industrially Relevant Conditions. Journal of Microelectromechanical Systems 19, 254-264 (2010). 
96. L. Liu et al., Evolution and stabilization of subnanometric metal species in confined space by in situ TEM. Nat Commun 9, 574 (2018).

97. J. E. Evans, K. L. Jungjohann, N. D. Browning, I. Arslan, Controlled growth of nanoparticles from solution with in situ liquid transmission electron microscopy. Nano Lett 11, 2809-2813 (2011).

98. T. J. Woehl et al., Direct observation of aggregative nanoparticle growth: kinetic modeling of the size distribution and growth rate. Nano Lett 14, 373-378 (2014).

99. P. Abellan et al., Gaining Control over Radiolytic Synthesis of Uniform Sub-3nanometer Palladium Nanoparticles: Use of Aromatic Liquids in the Electron Microscope. Langmuir 32, 1468-1477 (2016).

100. Y. Liu, K. Tai, S. J. Dillon, Growth Kinetics and Morphological Evolution of ZnO Precipitated from Solution. Chemistry of Materials 25, 2927-2933 (2013).

101. P. Abellan et al., The formation of cerium(iii) hydroxide nanoparticles by a radiation mediated increase in local pH. RSC Advances 7, 3831-3837 (2017).

102. N. M. Schneider et al., Electron-Water Interactions and Implications for Liquid Cell Electron Microscopy. The Journal of Physical Chemistry C 118, 2237322382 (2014).

103. P. Abellan et al., Factors influencing quantitative liquid (scanning) transmission electron microscopy. Chem Commun (Camb) 50, 4873-4880 (2014).

104. T. J. Woehl, P. Abellan, Defining the radiation chemistry during liquid cell electron microscopy to enable visualization of nanomaterial growth and degradation dynamics. J Microsc 265, 135-147 (2017).

105. T. J. Woehl, J. E. Evans, I. Arslan, W. D. Ristenpart, N. D. Browning, Direct in situ determination of the mechanisms controlling nanoparticle nucleation and growth. ACS Nano 6, 8599-8610 (2012).

106. N. Ahmad, G. Wang, J. Nelayah, C. Ricolleau, D. Alloyeau, Driving reversible redox reactions at solid-liquid interfaces with the electron beam of a transmission electron microscope. J Microsc 269, 127-133 (2018).

107. J. Zecevic, J. Hermannsdorfer, T. Schuh, K. P. de Jong, N. de Jonge, Anisotropic Shape Changes of Silica Nanoparticles Induced in Liquid with Scanning Transmission Electron Microscopy. Small 13, (2017).

108. M. S. A. Asghar, B. J. Inkson, G. Mobus, Giant Radiolytic Dissolution Rates of Aqueous Ceria Observed in Situ by Liquid-Cell TEM. Chemphyschem 18, 12471251 (2017). 
109. M. T. Proetto et al., Dynamics of soft nanomaterials captured by transmission electron microscopy in liquid water. J Am Chem Soc 136, 1162-1165 (2014).

110. M. Conroy et al., Importance of interlayer $\mathrm{H}$ bonding structure to the stability of layered minerals. Sci Rep 7, 13274 (2017).

111. U. M. Mirsaidov, H. Zheng, Y. Casana, P. Matsudaira, Imaging protein structure in water at $2.7 \mathrm{~nm}$ resolution by transmission electron microscopy. Biophys $J \mathbf{1 0 2}$, L15-17 (2012).

112. S. Kashyap, T. J. Woehl, X. Liu, S. K. Mallapragada, T. Prozorov, Nucleation of iron oxide nanoparticles mediated by Mms6 protein in situ. ACS Nano 8, 90979106 (2014).

113. T. Yamazaki et al., Two types of amorphous protein particles facilitate crystal nucleation. Proc Natl Acad Sci U S A 114, 2154-2159 (2017).

114. J. E. Evans et al., Visualizing macromolecular complexes with in situ liquid scanning transmission electron microscopy. Micron 43, 1085-1090 (2012).

115. Y. Wang et al., A Structural Study of Escherichia coli Cells Using an In Situ Liquid Chamber TEM Technology. J Anal Methods Chem 2015, 829302 (2015).

116. T. J. Woehl et al., Correlative electron and fluorescence microscopy of magnetotactic bacteria in liquid: toward in vivo imaging. Sci Rep 4, 6854 (2014).

117. E. Kennedy, E. M. Nelson, T. Tanaka, J. Damiano, G. Timp, Live Bacterial Physiology Visualized with $5 \mathrm{~nm}$ Resolution Using Scanning Transmission Electron Microscopy. ACS Nano 10, 2669-2677 (2016).

118. D. B. Peckys, P. Mazur, K. L. Gould, N. de Jonge, Fully hydrated yeast cells imaged with electron microscopy. Biophys $J$ 100, 2522-2529 (2011).

119. N. de Jonge, D. B. Peckys, G. J. Kremers, D. W. Piston, Electron microscopy of whole cells in liquid with nanometer resolution. Proc Natl Acad Sci U S A 106, 2159-2164 (2009).

120. D. B. Peckys, N. de Jonge, Visualizing gold nanoparticle uptake in live cells with liquid scanning transmission electron microscopy. Nano Lett 11, 1733-1738 (2011).

121. M. J. Dukes, R. Ramachandra, J. P. Baudoin, W. Gray Jerome, N. de Jonge, Three-dimensional locations of gold-labeled proteins in a whole mount eukaryotic cell obtained with $3 \mathrm{~nm}$ precision using aberration-corrected scanning transmission electron microscopy. J Struct Biol 174, 552-562 (2011). 
122. N. de Jonge, D. B. Peckys, Live Cell Electron Microscopy Is Probably Impossible. ACS Nano 10, 9061-9063 (2016).

123. E. Kennedy, E. M. Nelson, J. Damiano, G. Timp, Gene Expression in ElectronBeam-Irradiated Bacteria in Reply to "Live Cell Electron Microscopy Is Probably Impossible". ACS Nano 11, 3-7 (2017).

124. M. E. Holtz, Y. Yu, J. Gao, H. D. Abruna, D. A. Muller, In situ electron energyloss spectroscopy in liquids. Microsc Microanal 19, 1027-1035 (2013).

125. P. J. Smeets, K. R. Cho, R. G. Kempen, N. A. Sommerdijk, J. J. De Yoreo, Calcium carbonate nucleation driven by ion binding in a biomimetic matrix revealed by in situ electron microscopy. Nat Mater 14, 394-399 (2015).

126. J. H. Park et al., Control of Electron Beam-Induced Au Nanocrystal Growth Kinetics through Solution Chemistry. Nano Lett 15, 5314-5320 (2015).

127. N. Jiang, Note on in situ (scanning) transmission electron microscopy study of liquid samples. Ultramicroscopy 179, 81-83 (2017).

128. M. Madou, Fundamentals of Microfabrication: the Science of Miniaturization. (CRC Press, 2002).

129. R. Zaouk, B. Y. Park, M. J. Madou, Introduction to microfabrication techniques. Methods Mol Biol 321, 5-15 (2006).

130. D. C. Abeysinghe, S. Dasgupta, J. T. Boyd, H. E. Jackson, A novel MEMS pressure sensor fabricated on an optical fiber. IEEE Photonics Technology Letters 13, 993-995 (2001).

131. J. N. Palasagaram, R. Ramadoss, MEMS-Capacitive Pressure Sensor Fabricated Using Printed-Circuit-Processing Techniques. IEEE Sensors Journal 6, 13741375 (2006).

132. W. Li-Peng et al., Design, fabrication, and measurement of high-sensitivity piezoelectric microelectromechanical systems accelerometers. Journal of Microelectromechanical Systems 12, 433-439 (2003).

133. W. J. Jack, Microelectromechanical systems (MEMS): fabrication, design and applications. Smart Materials and Structures 10, 1115 (2001).

134. L. Wang, D. M. Sipe, Y. Xu, Q. Lin, A MEMS Thermal Biosensor for Metabolic Monitoring Applications. Journal of Microelectromechanical Systems 17, 318327 (2008). 
135. S. M. Radke, E. C. Alocilja, Design and fabrication of a microimpedance biosensor for bacterial detection. IEEE Sensors Journal 4, 434-440 (2004).

136. Y. Arntz et al., Label-free protein assay based on a nanomechanical cantilever array. Nanotechnology 14, 86 (2003).

137. M. A. Unger, H.-P. Chou, T. Thorsen, A. Scherer, S. R. Quake, Monolithic Microfabricated Valves and Pumps by Multilayer Soft Lithography. Science 288, 113 (2000).

138. Y.-C. Toh et al., A novel 3D mammalian cell perfusion-culture system in microfluidic channels. Lab on a Chip 7, 302-309 (2007).

139. U. Helmersson, M. Lattemann, J. Bohlmark, A. P. Ehiasarian, J. T. Gudmundsson, Ionized physical vapor deposition (IPVD): A review of technology and applications. Thin Solid Films 513, 1-24 (2006).

140. S. M. Rossnagel, D. Mikalsen, H. Kinoshita, J. J. Cuomo, Collimated magnetron sputter deposition. Journal of Vacuum Science \& Technology A: Vacuum, Surfaces, and Films 9, 261-265 (1991).

141. P. J. Kelly, R. D. Arnell, Magnetron sputtering: a review of recent developments and applications. Vacuum 56, 159-172 (2000).

142. I. C. Huang et al., $10 \mathrm{~nm}$ gap bowtie plasmonic apertures fabricated by modified lift-off process. Applied Physics Letters 109, (2016).

143. K. R. Williams, K. Gupta, M. Wasilik, Etch rates for micromachining processingpart II. Journal of Microelectromechanical Systems 12, 761-778 (2003).

144. C. Iliescu, J. Jing, F. E. H. Tay, J. Miao, T. Sun, Characterization of masking layers for deep wet etching of glass in an improved $\mathrm{HF} / \mathrm{HCl}$ solution. Surface and Coatings Technology 198, 314-318 (2005).

145. F. E. H. Tay, C. Iliescu, J. Jing, J. Miao, Defect-free wet etching through pyrex glass using Cr/Au mask. Microsystem Technologies 12, 935-939 (2006).

146. K. Bean, Anisotropic etching of silicon. IEEE Transactions on electron devices 25, (1978).

147. H. Jansen et al., BSM 7: RIE lag in high aspect ratio trench etching of silicon. Microelectronic Engineering 35, 45-50 (1997).

148. F. Marty et al., Advanced etching of silicon based on deep reactive ion etching for silicon high aspect ratio microstructures and three-dimensional micro- and nanostructures. Microelectronics Journal 36, 673-677 (2005). 
149. F. Niklaus, G. Stemme, J. Q. Lu, R. J. Gutmann, Adhesive wafer bonding. Journal of Applied Physics 99, (2006).

150. M. A. Schmidt, Wafer-to-wafer bonding for microstructure formation. Proceedings of the IEEE 86, 1575-1585 (1998).

151. C.-T. Ko, K.-N. Chen, Wafer-level bonding/stacking technology for 3D integration. Microelectronics Reliability 50, 481-488 (2010).

152. C. H. Tsau, S. M. Spearing, M. A. Schmidt, Fabrication of wafer-level thermocompression bonds. Journal of Microelectromechanical Systems 11, 641647 (2002).

153. C. H. Tsau, S. M. Spearing, M. A. Schmidt, Characterization of Wafer-Level Thermocompression Bonds. Journal of Microelectromechanical Systems 13, 963 971 (2004).

154. V. Dragoi, E. Cakmak, E. Pabo, in 2009 International Semiconductor Conference. (2009), vol. 1, pp. 215-218.

155. S. H. Christiansen, R. Singh, U. Gosele, Wafer Direct Bonding: From Advanced Substrate Engineering to Future Applications in Micro/Nanoelectronics. Proceedings of the IEEE 94, 2060-2106 (2006).

156. A. Plöß1, Wafer direct bonding: tailoring adhesion between brittle materials. Materials Science and Engineering: R: Reports 25, 1-88 (1999).

157. C. Gui, M. Elwenspoek, N. Tas, J. G. E. Gardeniers, The effect of surface roughness on direct wafer bonding. Journal of Applied Physics 85, 7448-7454 (1999).

158. H. Moriceau, F. Rieutord, C. Morales, A. M. Charvet, Surface plasma treatments enabling low temperature direct bonding. Microsystem Technologies 12, 378-382 (2005).

159. J. A. Thornton, The microstructure of sputter-deposited coatings. Journal of Vacuum Science \& Technology A: Vacuum, Surfaces, and Films 4, 3059-3065 (1986).

160. A. Hanneborg, M. Nese, P. Ohlckers, Silicon-to-silicon anodic bonding with a borosilicate glass layer. Journal of Micromechanics and Microengineering 1, 139 (1991).

161. M. Esashi, A. Nakano, S. Shoji, H. Hebiguchi, Low-temperature silicon-to-silicon anodic bonding with intermediate low melting point glan. Sensors and Actuators A: Physical 23, $931-934$ (1990). 
162. A. Berthold, L. Nicola, P. M. Sarro, M. J. Vellekoop, Glass-to-glass anodic bonding with standard IC technology thin films as intermediate layers. Sensors and Actuators A: Physical 82, 224-228 (2000).

163. S. Weichel, R. d. Reus, M. Lindahl, Silicon-to-silicon wafer bonding using evaporated glass. Sensors and Actuators A: Physical 70, 179-184 (1998).

164. A. D. Brooks, R. P. Donovan, C. A. Hardesty, Low-Temperature Electrostatic Silicon-to-Silicon Seals Using Sputtered Borosilicate Glass. Journal of The Electrochemical Society 119, (1972).

165. R. F. Wolffenbuttel, K. D. Wise, Low-temperature silicon wafer-to-wafer bonding using gold at eutectic temperature. Sensors and Actuators A: Physical 43, 223229 (1994).

166. Y. Lin et al., in TRANSDUCERS 2009 - 2009 International Solid-State Sensors, Actuators and Microsystems Conference. (2009), pp. 244-247.

167. E. Jing, B. Xiong, Y. Wang, Low-temperature Au/a-Si wafer bonding. Journal of Micromechanics and Microengineering 21, (2011).

168. Y. Endo, T. Sawasaki, Cell-free expression systems for eukaryotic protein production. Curr Opin Biotechnol 17, 373-380 (2006).

169. J. F. Zawada et al., Microscale to manufacturing scale-up of cell-free cytokine production--a new approach for shortening protein production development timelines. Biotechnol Bioeng 108, 1570-1578 (2011).

170. E. D. Carlson, R. Gan, C. E. Hodgman, M. C. Jewett, Cell-free protein synthesis: applications come of age. Biotechnol Adv 30, 1185-1194 (2012).

171. F. Katzen, G. Chang, W. Kudlicki, The past, present and future of cell-free protein synthesis. Trends Biotechnol 23, 150-156 (2005).

172. T. Sawasaki, T. Ogasawara, R. Morishita, Y. Endo, A cell-free protein synthesis system for high-throughput proteomics. Proc Natl Acad Sci U S A 99, 1465214657 (2002).

173. C. A. Kerfeld et al., Protein structures forming the shell of primitive bacterial organelles. Science 309, 936-938 (2005).

174. F. Cai, M. Sutter, S. L. Bernstein, J. N. Kinney, C. A. Kerfeld, Engineering bacterial microcompartment shells: chimeric shell proteins and chimeric carboxysome shells. ACS Synth Biol 4, 444-453 (2015). 
175. S. Tanaka et al., Atomic-level models of the bacterial carboxysome shell. Science 319, 1083-1086 (2008).

176. K. A. Dryden, C. S. Crowley, S. Tanaka, T. O. Yeates, M. Yeager, Twodimensional crystals of carboxysome shell proteins recapitulate the hexagonal packing of three-dimensional crystals. Protein Sci 18, 2629-2635 (2009).

177. M. Yeager, K. A. Dryden, B. K. Ganser-Pornillos, Lipid monolayer and sparse matrix screening for growing two-dimensional crystals for electron crystallography: methods and examples. Methods Mol Biol 955, 527-537 (2013).

178. D. Levy, M. Chami, J. L. Rigaud, Two-dimensional crystallization of membrane proteins: the lipid layer strategy. FEBS Lett 504, 187-193 (2001).

179. T. Braun, A. Engel, in Encyclopedia of Life Sciences. (2005).

180. R. K. Hite, S. Raunser, T. Walz, Revival of electron crystallography. Curr Opin Struct Biol 17, 389-395 (2007).

181. N. Biyani et al., Image processing techniques for high-resolution structure determination from badly ordered 2D crystals. J Struct Biol 203, 120-134 (2018).

182. B. Gipson, X. Zeng, H. Stahlberg, 2dx_merge: data management and merging for 2D crystal images. J Struct Biol 160, 375-384 (2007).

183. B. Gipson, X. Zeng, Z. Y. Zhang, H. Stahlberg, 2dx--user-friendly image processing for 2D crystals. J Struct Biol 157, 64-72 (2007).

184. N. Biyani et al., Focus: The interface between data collection and data processing in cryo-EM. J Struct Biol 198, 124-133 (2017).

185. C. Degraeve-Guilbault et al., Glycerolipid Characterization and Nutrient Deprivation-Associated Changes in the Green Picoalga Ostreococcus tauri. Plant Physiol 173, 2060-2080 (2017).

186. J. E. Chen, A. G. Smith, A look at diacylglycerol acyltransferases (DGATs) in algae. J Biotechnol 162, 28-39 (2012).

187. B. E. Bammes, J. Jakana, M. F. Schmid, W. Chiu, Radiation damage effects at four specimen temperatures from 4 to $100 \mathrm{~K}$. J Struct Biol 169, 331-341 (2010).

188. D. L. Dorset, D. F. Parsons, Electron diffraction from single, fully-hydrated, oxliver catalase microcrystals. Acta Crystallographica Section A 31, 210-215 (1975). 
189. T. P. Ko, J. Day, A. J. Malkin, A. McPherson, Structure of orthorhombic crystals of beef liver catalase. Acta Crystallogr D Biol Crystallogr 55, 1383-1394 (1999).

190. V. R. Matricardi, R. C. Moretz, D. F. Parsons, Electron diffraction of wet proteins: catalase. Science 177, 268-270 (1972).

191. J. B. Sumner, A. L. Dounce, Crystaline catalase. Journal of Biological Chemistry 121, 417-424 (1937).

192. S. Berthet et al., Crystallization and preliminary structural analysis of catalase A from Saccharomyces cerevisiae. Protein Sci 6, 481-483 (1997).

193. R. M. Glaeser, Specimen Behavior in the Electron Beam. Methods Enzymol 579, 19-50 (2016).

194. H. H. Mollenhauer, Artifacts caused by dehydration and epoxy embedding in transmission electron microscopy. Microsc Res Tech 26, 496-512 (1993).

195. E. I. Tocheva, Z. Li, G. J. Jensen, Electron cryotomography. Cold Spring Harb Perspect Biol 2, a003442 (2010).

196. S. Asano, B. D. Engel, W. Baumeister, In Situ Cryo-Electron Tomography: A Post-Reductionist Approach to Structural Biology. J Mol Biol 428, 332-343 (2016).

197. E. Cserti et al., Dynamics of the peptidoglycan biosynthetic machinery in the stalked budding bacterium Hyphomonas neptunium. Mol Microbiol 103, 875-895 (2017).

198. L. A. Baker, J. L. Rubinstein, Radiation damage in electron cryomicroscopy. Methods Enzymol 481, 371-388 (2010).

199. C. S. Ting, C. Hsieh, S. Sundararaman, C. Mannella, M. Marko, Cryo-electron tomography reveals the comparative three-dimensional architecture of Prochlorococcus, a globally important marine cyanobacterium. J Bacteriol 189, 4485-4493 (2007).

200. G. P. Henderson, L. Gan, G. J. Jensen, 3-D ultrastructure of O. tauri: electron cryotomography of an entire eukaryotic cell. PLoS One 2, e749 (2007).

201. M. Wagner et al., Identification and characterization of an acylCoA:diacylglycerol acyltransferase 2 (DGAT2) gene from the microalga $\mathrm{O}$. tauri. Plant Physiol Biochem 48, 407-416 (2010).

202. I. Khozin-Goldberg, Z. Cohen, Unraveling algal lipid metabolism: Recent advances in gene identification. Biochimie 93, 91-100 (2011). 
203. D. H. Nies, Efflux-mediated heavy metal resistance in prokaryotes. FEMS Microbiology Reviews 27, 313-339 (2003).

204. P. Monsieurs et al., Heavy metal resistance in Cupriavidus metallidurans CH34 is governed by an intricate transcriptional network. Biometals 24, 1133-1151 (2011).

205. P. J. Janssen et al., The complete genome sequence of Cupriavidus metallidurans strain $\mathrm{CH} 34$, a master survivalist in harsh and anthropogenic environments. PLoS One 5, e10433 (2010).

206. L. Fairbrother et al., Biomineralization of gold in biofilms of Cupriavidus metallidurans. Environ Sci Technol 47, 2628-2635 (2013).

207. F. Reith, S. L. Rogers, D. C. McPhail, D. Webb, Biomineralization of gold: biofilms on bacterioform gold. Science 313, 233-236 (2006).

208. F. Reith et al., Mechanisms of gold biomineralization in the bacterium Cupriavidus metallidurans. Proc Natl Acad Sci U S A 106, 17757-17762 (2009).

209. H. S. Marie et al., Bio-distribution and in vivo antioxidant effects of cerium oxide nanoparticles in mice. Environmental Toxicology 28, 107-118 (2013).

210. R. W. Tarnuzzer, J. Colon, S. Patil, S. Seal, Vacancy Engineered Ceria Nanostructures for Protection from Radiation-Induced Cellular Damage. Nano Letters 5, 2573-2577 (2005).

211. L. Wang, Q. Luan, D. Yang, X. Yao, K. Zhou, Strong electron-conjugation interaction facilitates electron transfer of hemoglobin by $\mathrm{Ce}(\mathrm{OH}) 3$ nanorods. $R S C$ Advances 3, 6339-6342 (2013).

212. E. Grulke et al., Nanoceria: factors affecting its pro- and anti-oxidant properties. Environmental Science: Nano 1, 429-444 (2014).

213. A. Kumar et al., Behavior of nanoceria in biologically-relevant environments. Environmental Science: Nano 1, 516-532 (2014).

214. B. Pastina, J. A. LaVerne, Effect of Molecular Hydrogen on Hydrogen Peroxide in Water Radiolysis. The Journal of Physical Chemistry A 105, 9316-9322 (2001).

215. N. M. Schneider et al., Electron-Water Interactions and Implications for Liquid Cell Electron Microscopy. J Phys Chem C 118, 22373-22382 (2014).

216. K. B. James, P. M. George, L. Ning, The G value in plasma and radiation chemistry. Journal of Applied Physics 68, 3661-3668 (1990). 
217. B. Pastina, J. A. LaVerne, Effect of molecular hydrogen on hydrogen peroxide in water radiolysis. J Phys Chem A 105, 9316-9322 (2001).

218. T. J. Woehl et al., Experimental procedures to mitigate electron beam induced artifacts during in situ fluid imaging of nanomaterials. Ultramicroscopy 127, 5363 (2013).

219. T. Schuh, N. de Jonge, Liquid scanning transmission electron microscopy: Nanoscale imaging in micrometers-thick liquids. Comptes Rendus Physique 15, 214-223 (2014).

220. J. E. Evans et al., Low-dose aberration corrected cryo-electron microscopy of organic specimens. Ultramicroscopy 108, 1636-1644 (2008).

221. J. Hosoi, T. Matsuo, Y. Ishida, Y. Harada, Process of irradiation damage to negatively stained helical structure observed by low dose method. J Electron Microsc (Tokyo) 30, 1-7 (1981).

222. C. Park, J. Z. Huang, J. X. Ji, Y. Ding, Segmentation, Inference and Classification of Partially Overlapping Nanoparticles. IEEE Trans Pattern Anal Mach Intell 35, 669-681 (2013).

223. C. Park, T. J. Woehl, J. E. Evans, N. D. Browning, Minimum Cost Multi-Way Data Association for Optimizing Multitarget Tracking of Interacting Objects. IEEE Trans Pattern Anal Mach Intell 37, 611-624 (2015).

224. A. F. Brilot et al., Beam-induced motion of vitrified specimen on holey carbon film. J Struct Biol 177, 630-637 (2012).

225. E. Sutter et al., In situ liquid-cell electron microscopy of silver-palladium galvanic replacement reactions on silver nanoparticles. Nat Commun 5, 4946 (2014).

226. Y. Kimura, H. Niinomi, K. Tsukamoto, J. M. Garcia-Ruiz, In situ live observation of nucleation and dissolution of sodium chlorate nanoparticles by transmission electron microscopy. J Am Chem Soc 136, 1762-1765 (2014).

227. S. A. Canepa, B. T. Sneed, H. Sun, R. R. Unocic, K. Mølhave, Influence of Cetyltrimethylammonium Bromide on Gold Nanocrystal Formation Studied by In Situ Liquid Cell Scanning Transmission Electron Microscopy. The Journal of Physical Chemistry C 122, 2350-2357 (2018).

228. J. T. McKeown, Y. Wu, J. D. Fowlkes, P. D. Rack, G. H. Campbell, Simultaneous in-situ synthesis and characterization of $\mathrm{Co} @ \mathrm{Cu}$ core-shell nanoparticle arrays. Adv Mater 27, 1060-1065 (2015). 
229. N. T. Thanh, N. Maclean, S. Mahiddine, Mechanisms of nucleation and growth of nanoparticles in solution. Chem Rev 114, 7610-7630 (2014).

230. Y. Xia, Y. Xiong, B. Lim, S. E. Skrabalak, Shape-controlled synthesis of metal nanocrystals: simple chemistry meets complex physics? Angew Chem Int Ed Engl 48, 60-103 (2009).

231. I. M. Lifshitz, V. V. Slyozov, The kinetics of precipitation from supersaturated solid solutions. Journal of Physics and Chemistry of Solids 19, 35-50 (1961).

232. R. M. Glaeser, G. McMullan, A. R. Faruqi, R. Henderson, Images of paraffin monolayer crystals with perfect contrast: minimization of beam-induced specimen motion. Ultramicroscopy 111, 90-100 (2011).

233. M. Karuppasamy, F. Karimi Nejadas1, M. Vulovic, A. J. Koster, R. B. Ravelli, Radiation damage in single-particle cryo-electron microscopy: effects of dose and dose rate. J Synchrotron Radiat 18, 398-412 (2011).

234. R. M. Glaeser, Retrospective: radiation damage and its associated "information limitations". J Struct Biol 163, 271-276 (2008).

235. C. Mueller, M. Harb, J. R. Dwyer, R. J. D. Miller, Nanofluidic Cells with Controlled Pathlength and Liquid Flow for Rapid, High-Resolution In Situ Imaging with Electrons. The Journal of Physical Chemistry Letters 4, 2339-2347 (2013).

236. C. Gui, M. Elwenspoek, N. Tas, Gardeniers, The effect of surface roughness on direct wafer bonding. Journal of Applied Physics 85, (1999).

237. S. M. L. Nai, J. Wei, P. C. Lim, C. K. Wong, in Proceedings of the 5th Electronics Packaging Technology Conference (EPTC 2003). (2003), pp. 119124.

238. R. F. Wolffenbuttel, Low-temperature intermediate Au-Si wafer bonding; eutectic or silicide bond. Sensors and Actuators A: Physical 62, 680-686 (1997).

239. T. H. Moser et al., The role of electron irradiation history in liquid cell transmission electron microscopy. Sci Adv 4, eaaq1202 (2018).

240. N. de Jonge, Theory of the spatial resolution of (scanning) transmission electron microscopy in liquid water or ice layers. Ultramicroscopy 187, 113-125 (2018).

241. A. A. Sousa, M. F. Hohmann-Marriott, G. Zhang, R. D. Leapman, Monte Carlo electron-trajectory simulations in bright-field and dark-field STEM: implications for tomography of thick biological sections. Ultramicroscopy 109, 213-221 (2009). 
242. K. Aoyama, K. Nagano, K. Mitsuoka, Optimization of STEM imaging conditions for cryo-tomography. Microscopy (Oxf) 66, 212-216 (2017).

243. K. Iakoubovskii, K. Mitsuishi, Y. Nakayama, K. Furuya, Thickness measurements with electron energy loss spectroscopy. Microsc Res Tech 71, 626631 (2008).

244. K. L. Jungjohann, J. E. Evans, J. A. Aguiar, I. Arslan, N. D. Browning, Atomicscale imaging and spectroscopy for in situ liquid scanning transmission electron microscopy. Microsc Microanal 18, 621-627 (2012).

245. J. T. Thong, K. S. Sim, J. C. Phang, Single-image signal-to-noise ratio estimation. Scanning 23, 328-336 (2001).

246. D. A. Welch, R. Faller, J. E. Evans, N. D. Browning, Simulating realistic imaging conditions for in situ liquid microscopy. Ultramicroscopy 135, 36-42 (2013).

247. J. Hattne et al., Analysis of Global and Site-Specific Radiation Damage in CryoEM. Structure 26, 759-766 e754 (2018).

248. L. A. Baker, E. A. Smith, S. A. Bueler, J. L. Rubinstein, The resolution dependence of optimal exposures in liquid nitrogen temperature electron cryomicroscopy of catalase crystals. $J$ Struct Biol 169, 431-437 (2010). 